\title{
A qualitative study to investigate the barriers to adoption of a lifestyle associated with optimal peak bone mass acquisition
}

A Master of Clinical Research Thesis

Presented to

The Faculty of Science, School of Biological Sciences

Victoria University of Wellington, NZ

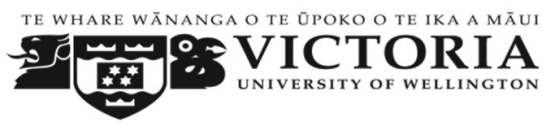

In Partial Fulfillment

of the requirements for the degree

Master of Clinical Research

By

Dr. Sana Zafar

under supervision of Professor Elaine Dennison

September 2019 


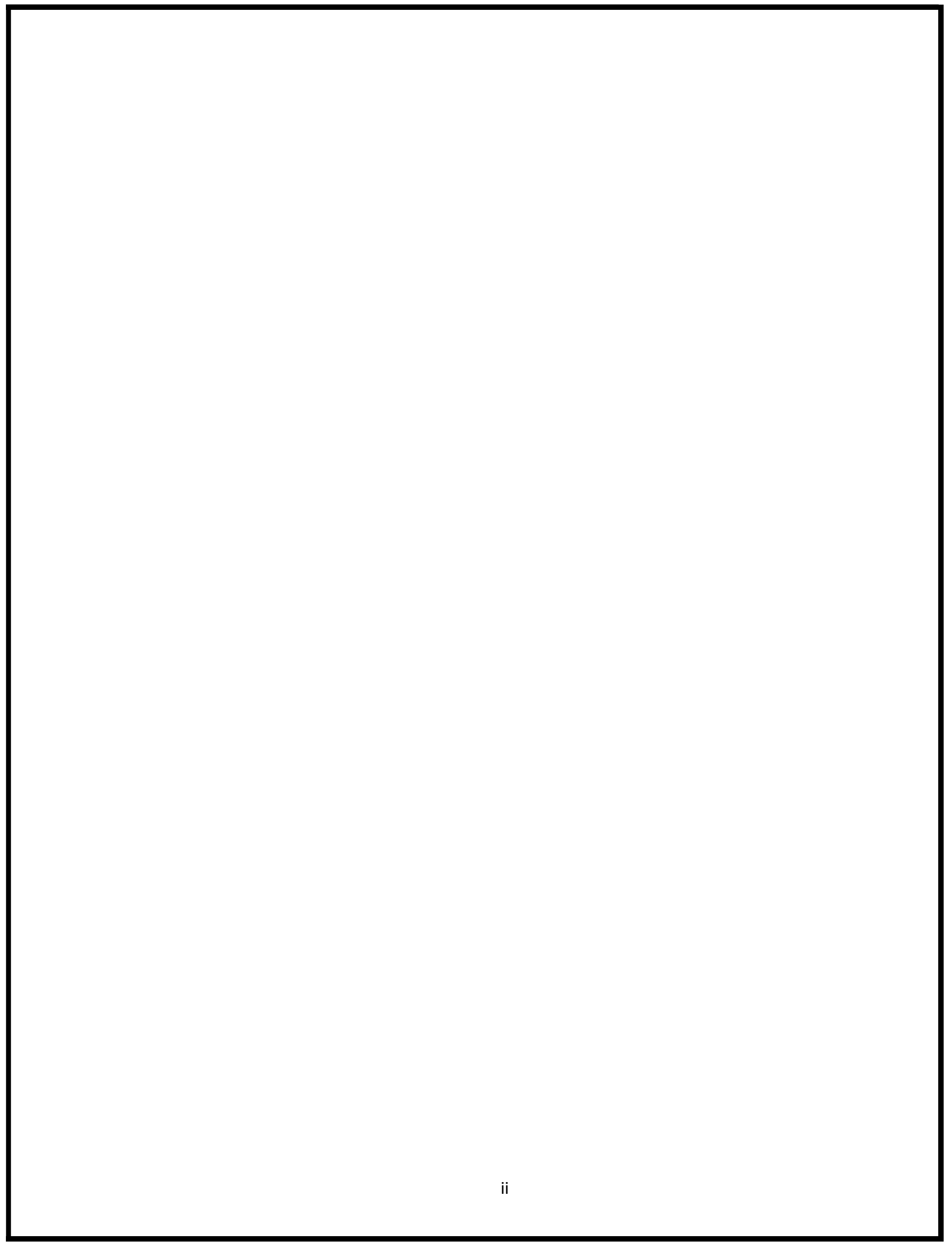




\section{Abstract}

\section{Background}

Osteoporosis is a major public health problem through its association with fragility fracture. Peak bone mass is attained in the second or third decade and has been shown to be a major determinant of later osteoporosis risk. Important determinants of peak bone mass include weight bearing physical activity levels, diet, cigarette smoking, and alcohol consumption.

\section{$\underline{\text { Aim }}$}

This study aimed to elucidate knowledge of factors affecting bone health among young people and identify lifestyle barriers to attainment of peak bone mass.

\section{Methods}

Participants were recruited through mass mailing of University students and staff aged 18-35 at Victoria University of Wellington. Six semi structured focus group interviews were conducted, where knowledge of factors associated with bone health and attitudes to lifestyles associated with beneficial health behaviors were explored. The interviews were audio taped and transcribed. A thematic approach for data analysis using constant comparative method was performed with Nvivo software. 


\section{$\underline{\text { Results }}$}

A total of 28 students ( 7 males, 21 females, mean age 28 years) were included. Seven themes emerged with regard to knowledge about factors affecting bone health which included physical activity, dairy, menopause, aging, smoking, alcohol and lack of knowledge about osteoporosis. For barriers, broadly eight themes of lifestyle factors emerged that included time, lack of resources, student life, cost, weather, cultural factors, lack of motivation and lastly smoking and alcohol. Students had limited knowledge about bone health in general, prevention of osteoporosis, and the importance of weight bearing physical activity and diet in determining later bone health. Some participants, especially vegans, expressed difficulties with making diet choices adequate in calcium, while others reported time management was a barrier to incorporating physical activity into everyday life. A few voiced a lack of motivation to exercise in bad weather. Some participants reported behaviors detrimental to bone health such as smoking and heavy alcohol consumption, with peer pressure being one factor leading to an increase in such activities, compounded by cultural and social pressures. Many students aspired to a healthier lifestyle but felt that they had limited knowledge about bone health, and specifically what they might need do to improve it.

\section{Conclusions}

These data highlights a lack of awareness of factors that impact peak bone mass among University students, an expressed desire to know more, and a keenness to adopt healthier behaviors. School-based education could provide stronger foundations with regards to knowledge of bone health. These observational data might help design interventions that encourage optimal peak bone mass in later life. 


\section{Acknowledgements}

There are many people I would like to thank, without whom it would not be possible to complete this thesis.

- I would like to acknowledge my supervisor Prof. Elaine Dennison for providing her expert advice, guidance and constant support though out writing this thesis. Thank you for providing me with such great opportunities and being a source of inspiration.

- To my co-supervisor Hayley Denison. Thank you for teaching me about qualitative study and for being so supportive and helpful throughout this journey.

- To my husband Mohammed Firasat. Thank you for your unconditional love, support and help with the children. Without you, this would have not been possible. Thank you for understanding of my need and love for learning.

- To my parents. Thank you for everything, you made me into who I am.

- Lastly to all the wonderful staff of Victoria University for helping generously. 


\section{Dedications}

Dedicated to my children Shayaan Ali and Azhaan Ali. 


\section{Abbreviations}

List of Abbreviations used: in alphabetical order

aBMD Areal Bone Mineral Density

BMC Bone Mineral Content

BMD Bone Mineral Density

BMI Body Mass Index

BMPs Bone Morphogenetic Proteins

BONZ Burden of Osteoporosis in New Zealand

CLAL The Global Dairy Market

CTSK Cathepsin K

DEXA Dual Energy X-ray Absorptiometry

EVOS European Vertebral Osteoporosis Study

FGF23 Fibroblast Growth Factor 23

FGFs Fibroblast Growth Factors

FM Fat Mass

GHO Global Health Observatory

GPRD General Practice Research Database

HED Heavy Episode Drinking

IGF-1 Insulin-like Growth Factor 1

LM Lean Mass

MMP9 Matrix Metallopeptidase 9

MPLA Minimum Legal Purchase Age

NF-kB Nuclear Factor Kappa-light-chain-enhancer of activated B cells

OCPs Oral Contraceptive Pills 
OPG Osteoprotegerin

PTH Parathyroid Hormone

PTH1Rs Parathyroid hormone receptor 1

QALYS Quality-Adjusted Life Years

RANK Receptor Activator for Nuclear Factor-KB

RANKL Receptor Activator for Nuclear Factor-KB Ligand

SENECA Survey in Europe on Nutrition and the Elderly; a Concerted Action

SHBG Sex Hormone Binding Globulin

TGF-B Transforming Growth Factor Beta

TNF Tumor Necrosis Factor

Uni University

USPSTF United States Preventive Services Task Force

VDR Vitamin D Receptor

WHO World Health Organization 


\section{Contents}

Abstract. iii

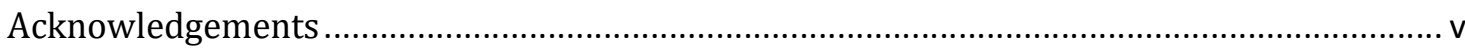

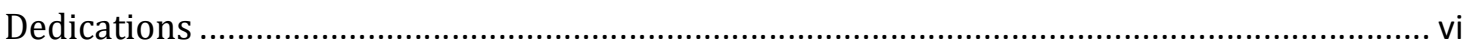

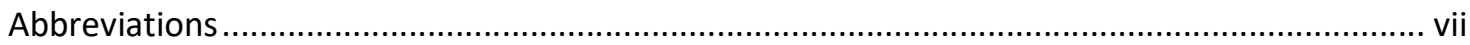

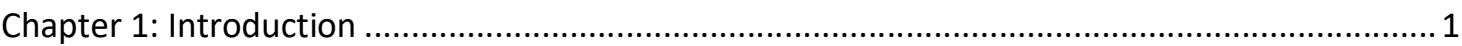

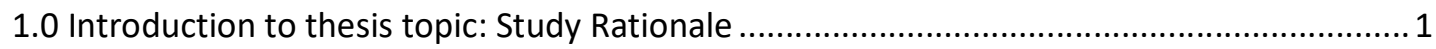

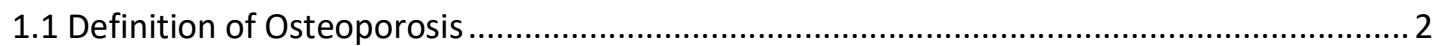

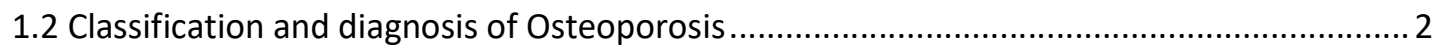

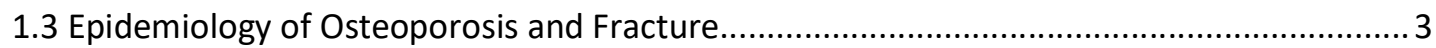

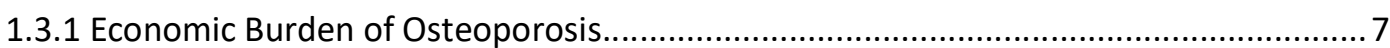

1.3.2 Morbidity and mortality associated with osteoporosis...............................................

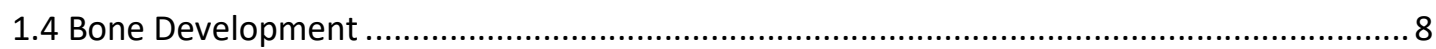

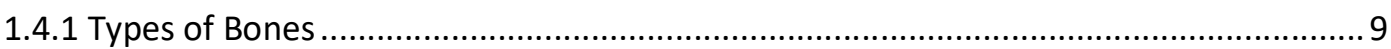

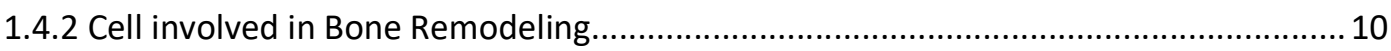

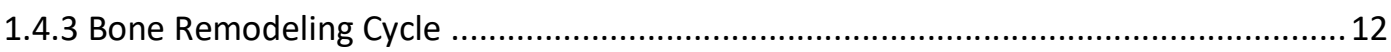

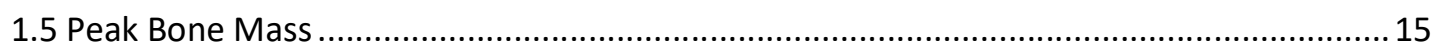

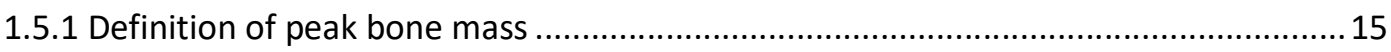

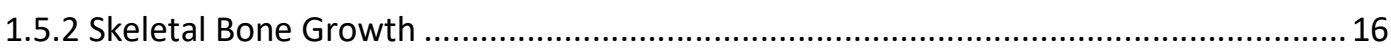

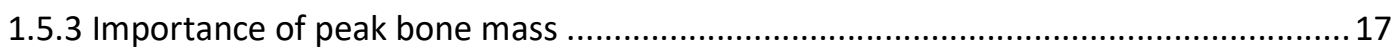

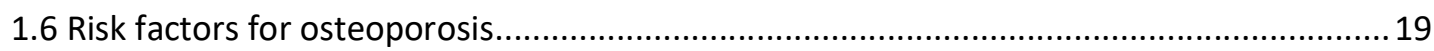

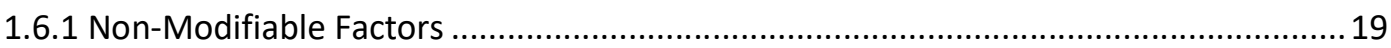

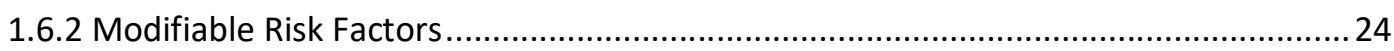

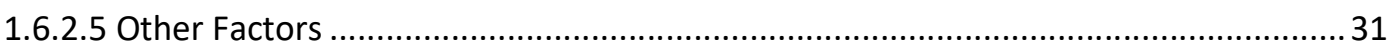

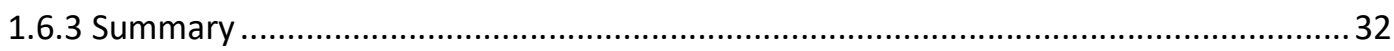

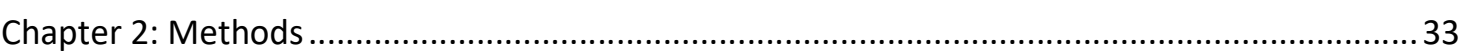

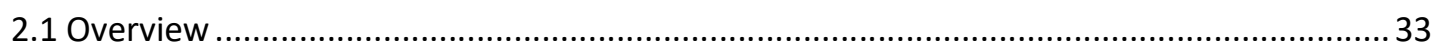

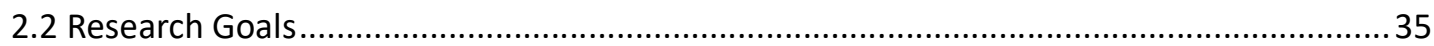

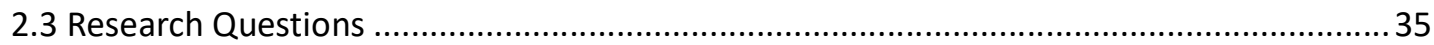

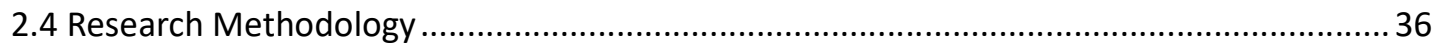

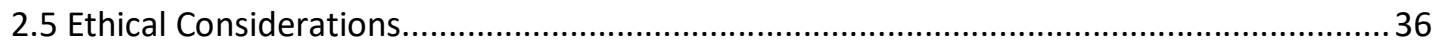

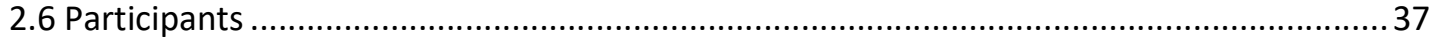


2.7 Data Collection

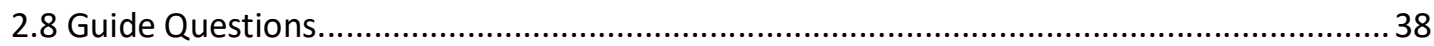

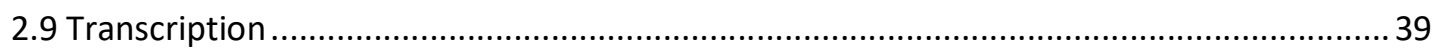

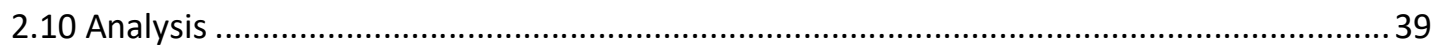

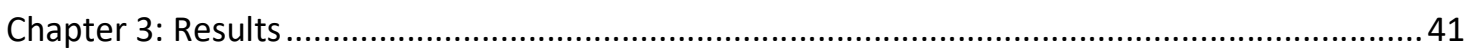

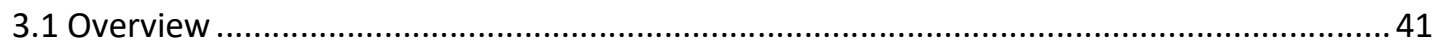

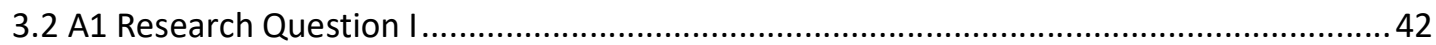

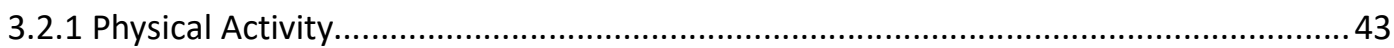

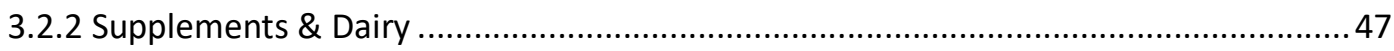

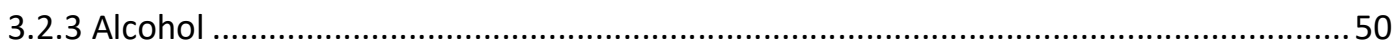

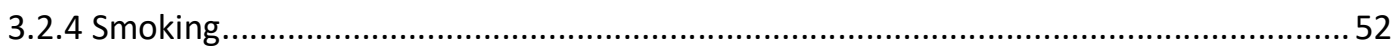

3.2.5 Lack of Knowledge to sequelae of poor bone health .............................................53

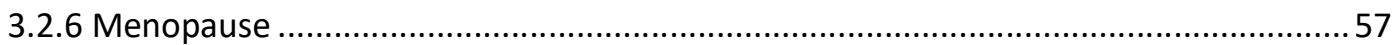

3.2.7 Aging \& Medical IIIness ......................................................................... 58

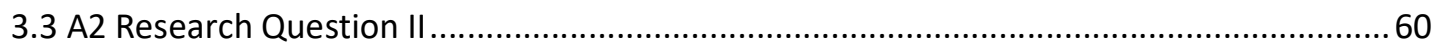

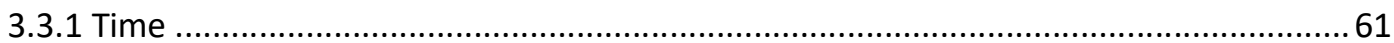

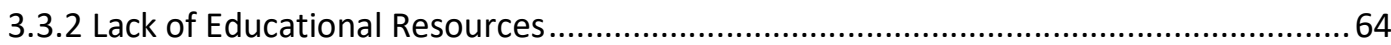

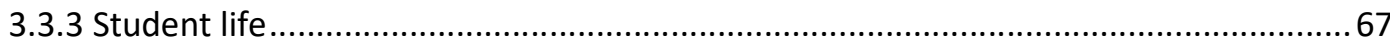

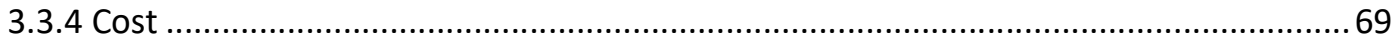

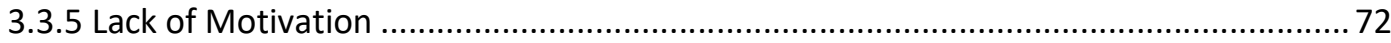

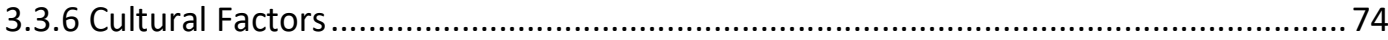

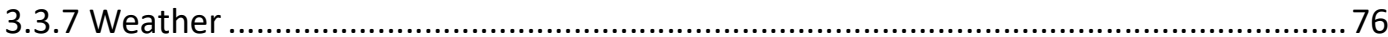

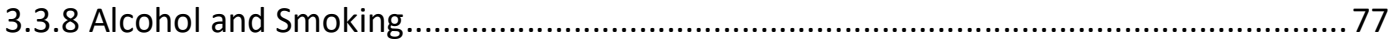

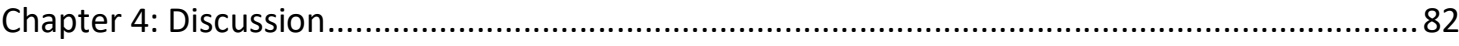

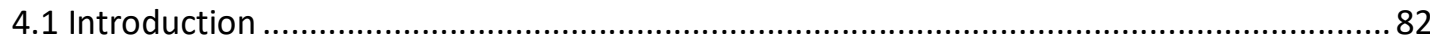

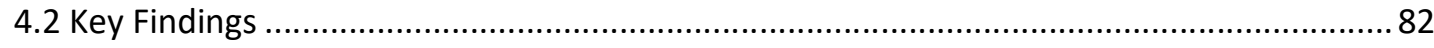

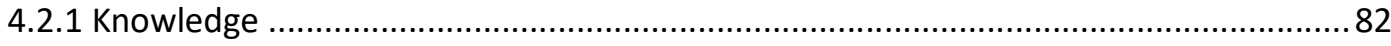

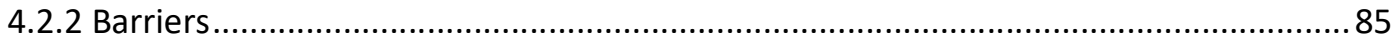

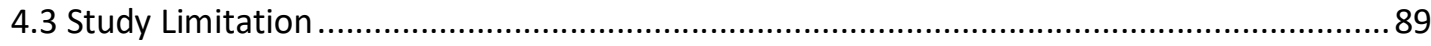

4.4 Adolescents and bone health: the importance of this work .......................................90

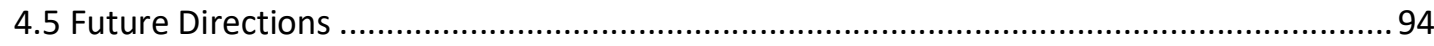

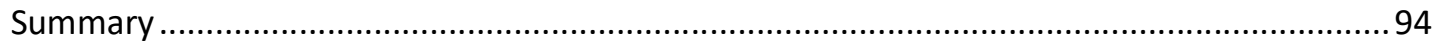




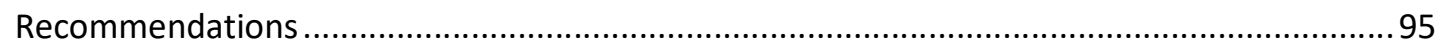

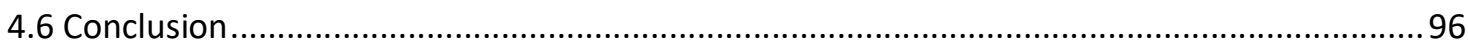

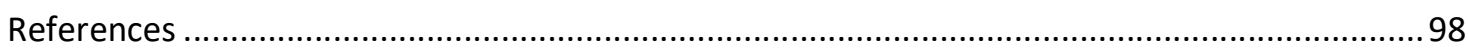

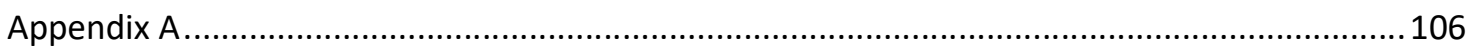

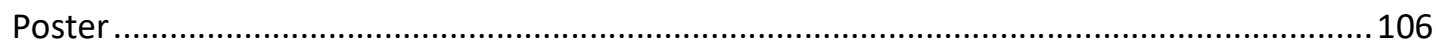

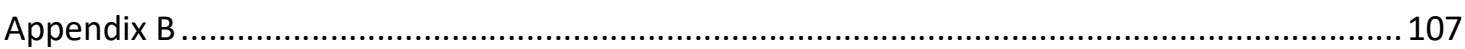

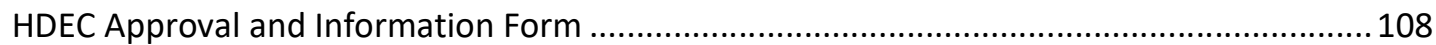

HDEC Approval: The ethics for this study were obtained as part of a larger existing study ... 108 INFORMATION SHEET FOR PARTICIPANTS AGED 16 TO 35 INCLUSIVELY.................................... 111

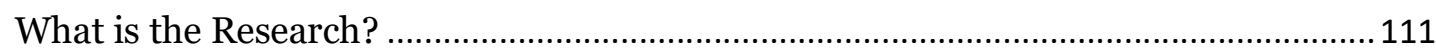

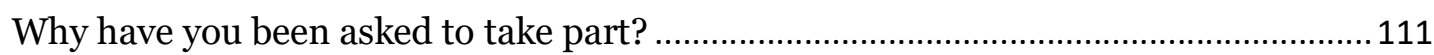

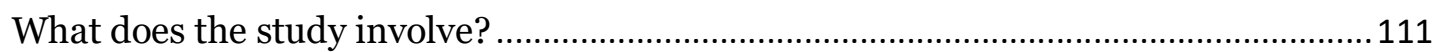

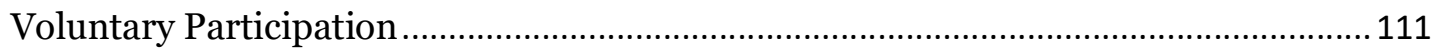

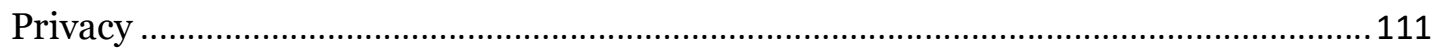

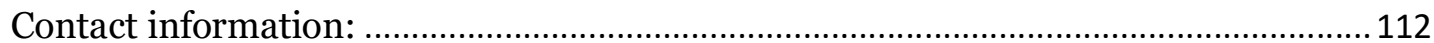

WRITTEN CONSENT TO PARTICIPATE FOR PARTICIPANTS AGED 16 to 35

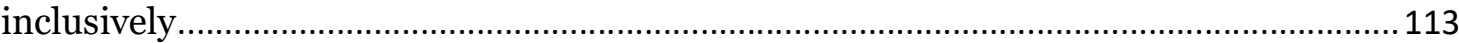

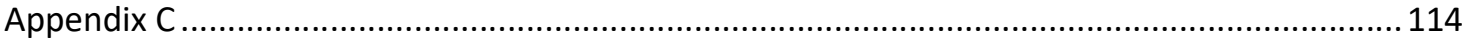

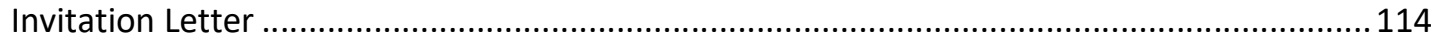

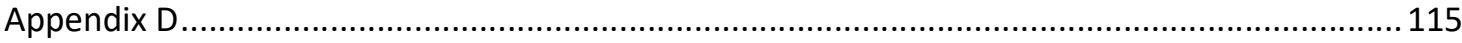

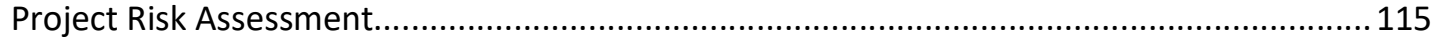




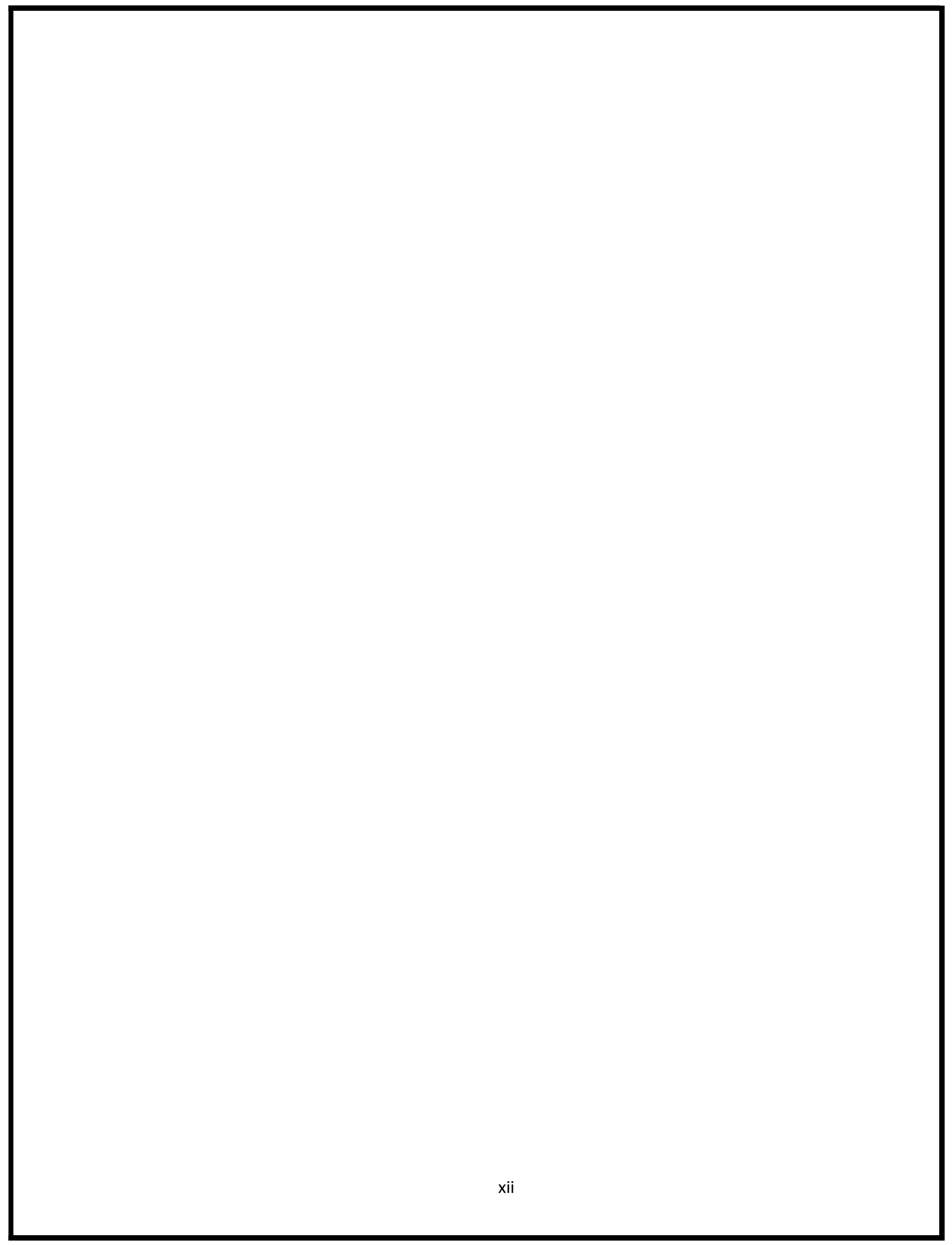




\section{Chapter 1: Introduction}

\subsection{Introduction to thesis topic: Study Rationale}

Osteoporosis is a major public health problem through its association with fragility fracture(1). The lifestyle we choose has a great impact on our subsequent bone health. The availability of fast food, and the rising obesity epidemic, linked to lower levels of weight bearing physical activity, especially in young adults, may lead to poorer bone health in late adulthood, at a time when fragility fracture is becoming more common. This occurs as a consequence of failure to attain a high peak bone mass, and the adoption of lifestyle that carries through to later life.

Our study's main aim was to assess the understanding and perception about bone health and the risk of fracture later in life among young adults and to investigate barriers faced that might impair ability to adopt a lifestyle associated with attainment of optimal peak bone mass. We chose to do this study because few studies have been conducted to date, looking into attitudes of young adolescents that might result in better bone health. A Canadian qualitative study was recently conducted which looked into barriers and enablers for achieving adequate calcium intake in young adults(2). They found that young adults had a limited knowledge of sources, prevention of disease and recommended dietary calcium amounts, and reflected on a lack of awareness relating to inadequacy and health consequences. Another study by Holland et al (2017) compared measured calcium and vitamin D intakes with perceptions of intake in Canadian young adults to design an osteoporosis prevention program and found that the majority of participants perceived they were consuming adequate amounts of calcium and vitamin $D$, when they were not(3).

To our knowledge, nobody has looked at knowledge and barriers for attaining a better peak bone mass, which can have a significant impact on reducing osteoporosis risk in later life. Our study explores attitudes to dietary calcium intake, but also considers other 
factors such as physical activity, smoking and alcohol intake, as well as exploring knowledge of bone health factors.

\subsection{Definition of Osteoporosis}

Osteoporosis is a skeletal bone disease which is caused by decreased bone density with deterioration of bone micro-architecture, resulting in decrease of both bone mass and volume. This leads to bone fragility and increase susceptibility to fractures(1). Osteoporosis can be defined as type 1, type 2 or secondary. Type 1 osteoporosis is seen in post-menopausal females due to estrogen deficiency, while type 2 osteoporosis is age related, mostly seen in older individuals. Secondary causes of osteoporosis include hypercortisolism, hyperthyroidism, hyperparathyroidism, alcohol abuse, and immobilization(1). It can also be due to prolonged use of medications such as corticosteroids.

\subsection{Classification and diagnosis of Osteoporosis}

Measurement of BMD (Bone mineral density) using DEXA (Dual energy X-ray Absorptiometry) is considered the "gold standard" for diagnosis of osteoporosis. This method uses two different X-ray sources through soft tissue and bone and subtracts the soft tissue leaving an estimate of skeletal BMD. A WHO(World Health Organization) panel has proposed as the diagnostic criterion for osteoporosis the limit of 2.5 standard deviations below the mean values recorded for DEXA results in young healthy individuals of the same gender(4). 
Figure 1 : Classification of Osteoporosis[adapted from reference (4)]

Classification

Normal

Low bone mass(osteopenia)

\section{T-Score}

-1.0 or greater

Between -1.0 or greater

-2.5 and below

-2.5 and below + Fragility fracture

\subsection{Epidemiology of Osteoporosis and Fracture}

The 2004 report from the US Surgeon General highlighted the enormous burden of osteoporosis-related fractures(5). An estimated 10 million Americans over 50 years old have osteoporosis and there are around 1.5 million fragility fractures each year. Another 34 million people are at risk of osteoporotic fracture. A study of British fracture occurrence indicated that population risk is similar in the UK(6), with one in two women aged 50 years expected to have an osteoporosis-related fracture in their remaining lifetime; the figure for men is one in five. Worldwide there were an estimated 1.66 million hip fractures in 1990(7); about 1.19 million in women and 463,000 in men. The projections indicate that the number of hip fractures occurring in the world each year will rise from 1.66 million in 1990 to 6.26 million by 2050(7).

According to the The Burden of Osteoporosis in New Zealand (BONZ) report in 2007, approximately 118,000 New Zealanders may be affected by Osteoporosis by $2020(8)$. In another study published in the Medical Journal of Australia (Preventing osteoporosis: outcomes of the Australian Fracture Prevention Summit), approximately 2.2 million 
individuals in Australia are affected by osteoporosis(9). In the absence of public health interventions, the prevalence of osteoporosis related conditions projected for 2013 and 2020 show that the estimated number of osteoporotic fractures will increase by $15 \%$ between 2007 and 2003, and 30\% between 2007 and 2020(8).

Fracture incidence is bimodal in distribution, being more common in youth and the elderly. While in youth, long bone fractures are more common, usually associated with substantial trauma, especially in males. By contrast, in later life, especially women over age of 50 years, hip and vertebral fractures are twice as common as in men(10).

For vertebral fractures, data from the European Vertebral Osteoporosis Study (EVOS) study have shown that the age-standardized population prevalence across Europe was $12.2 \%$ for men and $12.0 \%$ for women aged 50 to 79 years(11). Previously it was believed that vertebral fractures were more common in men than women, but the EVOS data suggest that this is not the case at younger ages: the prevalence of deformities in 50 to 60 year old's is similar, if not higher in men, possibly because of a greater incidence of trauma(11). Wrist fractures show a different pattern of occurrence to hip and vertebral fractures, with a gradual increase in rates with age (Figure 2)(12). Rates are higher in women than men at older ages, with an incidence of 39.7 per 10,000 person years and 8.9 per 10,000 person-years respectively in the UK for individuals aged 50 years or older(12, 13). 
Figure 2 : Age and sex specific incidence rates of fracture at the femur/hip, radius/ulna, and spine[adapted from reference (12)]

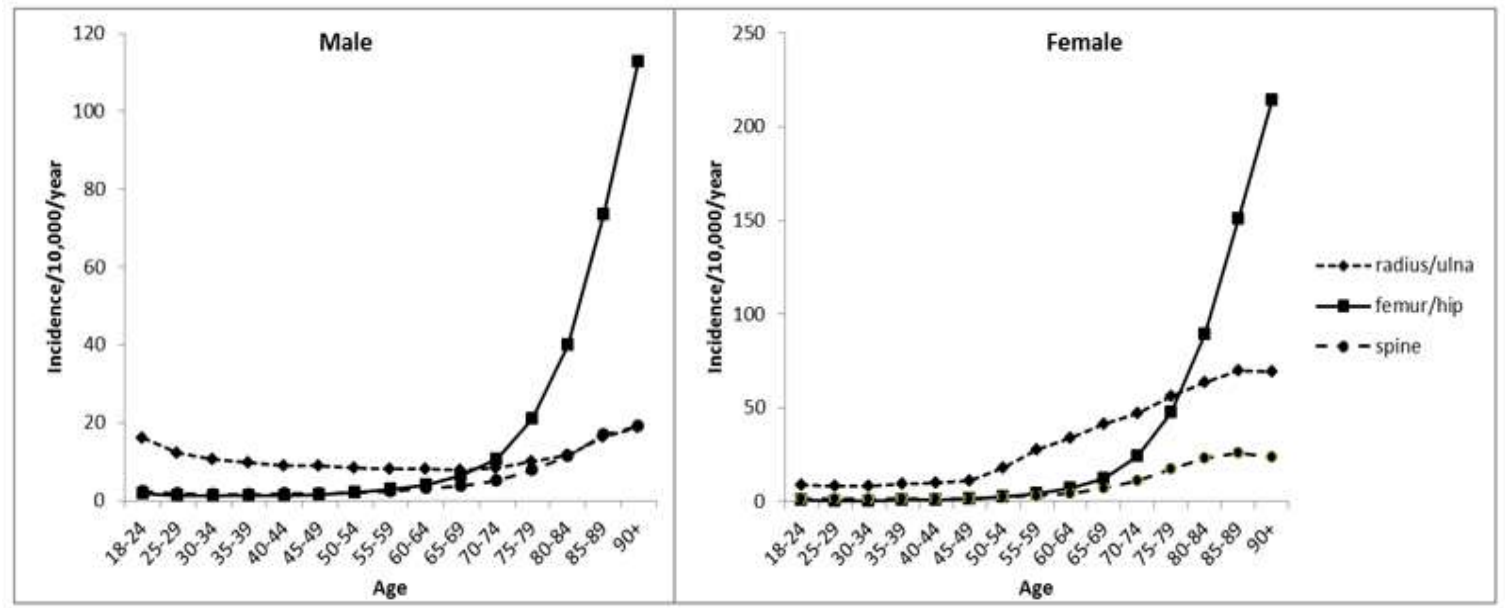

There are currently estimated to be 901 million people aged 60 years or over globally, comprising $12 \%$ of the world population. Although Europe has the greatest percentage of its population aged 60 years or older (24\%), rapid aging in other parts of the world means that by 2050 all major areas of the world except Africa will have nearly a quarter or more of their populations aged 60 years or older(13). This suggests that a very large number of people will be vulnerable to fragility fracture in the coming years. 
Figure 3 : Secular trends in hip fracture incidence worldwide[adapted from reference (14)]

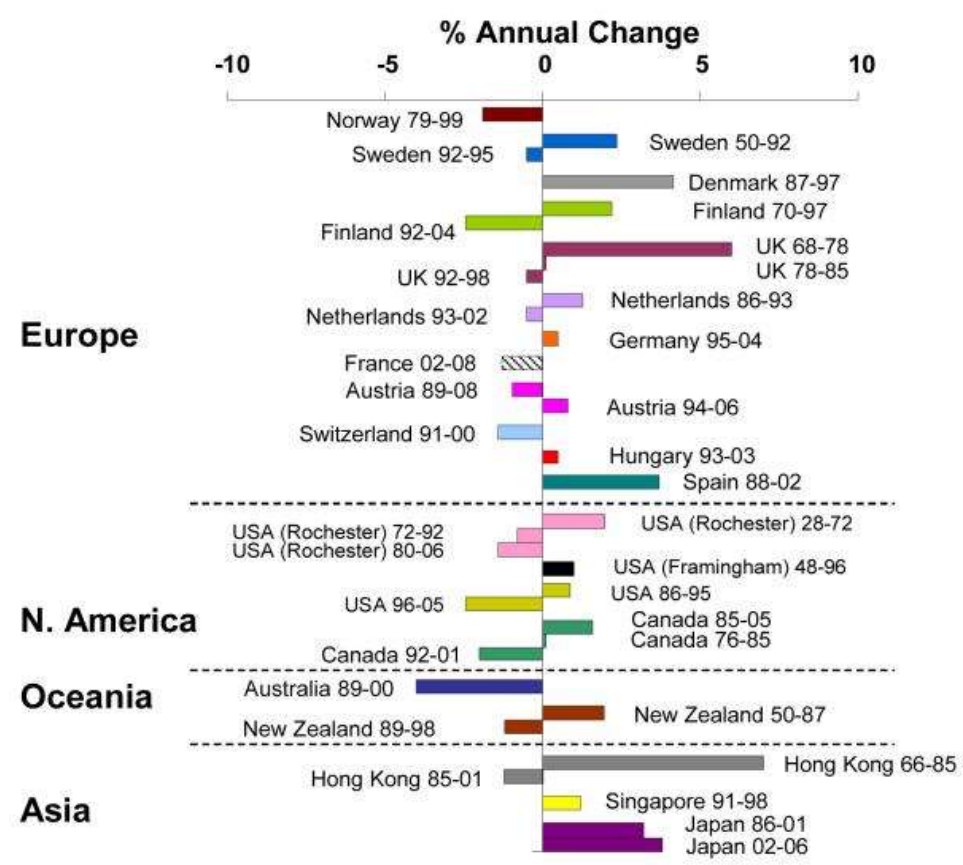

Scandinavia has the highest reported incidence of hip fracture worldwide(14) although the largest number of studies have been undertaken in UK. Age- and sex-specific fracture rates increased steadily over the first decade of this period but reached a plateau between 1979 and 1985 in the UK. Studies have also explored hip fracture incidence in Norway, Denmark and Finland. Using diagnostic registers maintained between 1979 and 1999, a similar plateau in the incidence of hip fracture has been observed in Oslo, Norway, to that seen in Sweden $(14,15)$. Hip fracture incidence rates in New Zealand and Australia have followed similar patterns to those observed in North America and Europe. A nationwide study in New Zealand observed significant increases in age-adjusted rates between 1950 and 1987(16) but a follow-up study using the New Zealand Health Information Service between 1988 and 1999 reported that the number of men and women aged 65 years and over with a hip fracture did not meet previous 
predictions $(14,17)$. The incidence of hip fracture in Singapore is now among the highest in Asia, and resembles that observed in Hong Kong, China (Figure 3).

\subsubsection{Economic Burden of Osteoporosis}

A study by Brown et al, 2011 estimated the economic burden of osteoporosis in New Zealand(18). There were an estimated 84354 osteoporotic fractures in New Zealand in 2007, including 3803 hip and 27994 vertebral fractures. Osteoporosis resulted in a loss of 11249 QALYs. The total direct cost of osteoporosis was \$NZ330 million, including \$NZ212 million to treat the fractures, \$NZ85 million for care after fractures and \$NZ34 million for treatment and management of the estimated 70631 people diagnosed with osteoporosis. The cost of treatment and management of osteoporosis is expected to increase to over \$NZ391 million in 2013 and \$NZ458 million in 2020, with the number of QALYs lost increasing to 13205 in 2013 and 15176 in 2020.

\subsubsection{Morbidity and mortality associated with osteoporosis}

Hip fracture mortality is higher in men than women, increases with age, and is greater for those with coexisting illnesses and poor pre-fracture functional status(19). There are around 31,000 excess deaths within 6 months of the approximately 300,000 hip fractures that occur annually in the USA. About $8 \%$ of men and $3 \%$ of women aged over 50 years die whilst hospitalized for their fracture. In the UK, the 12-month survival post-hip fracture for men is $63.3 \%$ versus $90.0 \%$ expected, and for women is $74.9 \%$ versus $91.1 \%$ expected(6).

Vertebral fractures are associated with increased mortality well beyond a year postfracture(20), with co-morbid conditions contributing significantly to the decreased relative survival. The impairment of survival following a vertebral fracture also markedly 
worsens as time from diagnosis of the fracture increases. In the UK General Practice Research Database (GPRD) study, the observed survival in women 12 months after vertebral fracture was $86.5 \%$ versus $93.6 \%$ expected. At 5 years, survival was $56.5 \%$ observed and $69.9 \%$ expected (6). By contrast, wrist fractures are not associated with an increase in mortality(6).

\subsection{Bone Development}

Bone is a type of dense connective tissue, which has a honeycomb like matrix internally to give bone rigidity yet keeping it flexible. Bone contains collagen which is a protein that provides a soft framework and calcium phosphate which adds strength and hardens the framework. Bone supports and protects various organs of the body and is a site of production of blood cells (Figure 4). It is also a reservoir for the body's calcium and phosphate minerals and provides structure and support to the body enabling mobility. Bone undergoes modeling and remodeling to grow and change its shape. It is a very dynamic organ which responds to changes due to physiological response or mechanical stress by remodeling. During childhood, bone undergoes both modeling, which includes the formation and shaping of bone, and remodeling, which includes replacing or renewing old bone whereas in adulthood, bone remodeling predominates(Figure 5)(21).

Figure 4 : Structure of Bone [adapted from reference(22)]

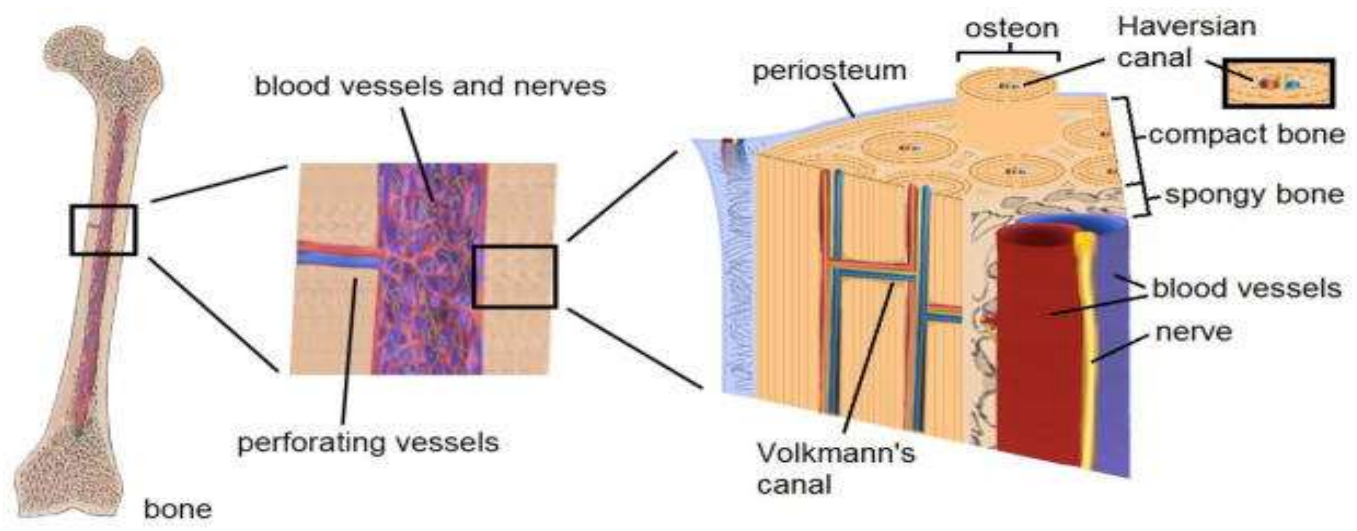




\subsubsection{Types of Bones}

There are 2 main types of bones that are distributed throughout the body. These are shown in Figure 4 above.

\subsubsection{Cortical bone}

The hard outer layer of bones is composed of cortical or compact bone. It forms the hard-exterior surface of bones and accounts for $80 \%$ of the total bone mass of an adult human skeleton. Closely packed haversian systems make up the cortical bone. The haversian system consists of a central canal called the haversian(osteonic) canal that is surrounded by concentric rings (lamellae) of matrix. There are bone cells (osteoblast and osteoclasts) located between the rings of the matrix. Bone cells are in the spaces called lacunae. The haversian canal contain blood vessels, lymphatic's, nerves and connective tissue which are parallel to long axis of bone (Figure 4).

\subsubsection{Trabecular bone}

Is also called cancellous bone. This is the internal tissue found in long skeletal bone and inner part of flat bones. It is less dense, softer, weaker, and less stiff. Trabecular bone has a higher surface-area-to-volume ratio than cortical bone because it is less dense. This makes it more flexible. The trabeculae of spongy bone follow the lines of stress and can realign if the direction of stress changes. It has an open cell porous network which contains bone marrow(Figure 4). 
Figure 5: Bone Remodeling Cycle [adapted from reference (23)]

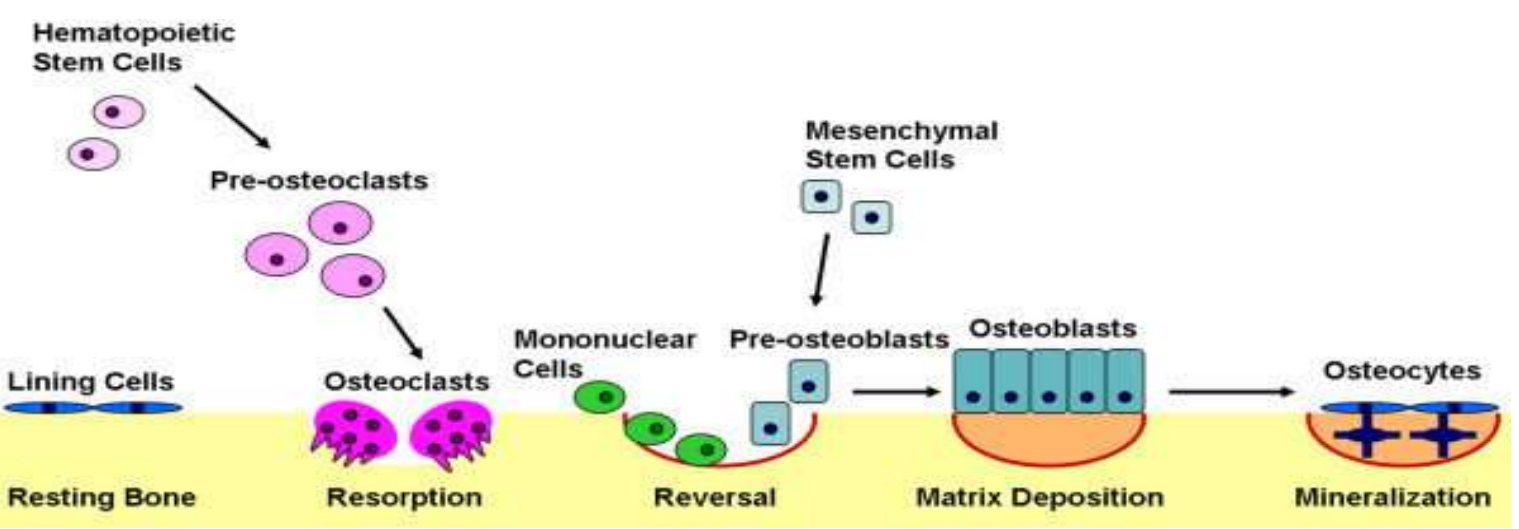

\subsubsection{Cell involved in Bone Remodeling}

The bone forming cells include osteoblast and osteocytes, while the bone resorption cells include osteoclast (precursor cells and mature osteoclasts). Bone is a very dynamic tissue and is in state of constant turnover. There is constant breakdown and buildup and this process is referred to as remodeling (Figure 5). The net effect of bone remodeling may be bone maintenance, bone loss or bone deposition. The balance is determined by osteoblasts which deposit bone and osteoclasts which resorb bone. This is balanced in health, but in some diseases and post-menopausal women, resorption exceeds formation leading to porous bones. In adolescence and young adulthood, the position is one of net formation.

\subsubsection{Osteoblasts}

Osteoblasts are the cells that form new bone. They are derived from mesenchymal stem cells, which are pluripotent cells. Osteoblasts work in teams to build bone. They produce new bone called "osteoid" by synthesizing dense cross-linked collagen and specialized proteins. They further mineralize the matrix by producing hydroxyapatite, 
which is deposited in a highly regulated manner forming a strong and dense mineralized tissue. They then control calcium and mineral deposition. They are found on the surface of the new bone. Osteoblasts have the potential to become fibroblasts, myoblasts, adipocytes or chondrocytes (Figure 6).

\subsubsection{Osteoclasts}

Osteoclasts are large cells that dissolve the bone. They are large, multinucleated cells that are derived from hematopoietic precursors of monocyte-macrophage lineage. They resorb mineralized bone. Many factors are involved in the development and activation of osteoclasts and which are discussed below (Figure 6).

\subsubsection{Osteocytes}

Osteocytes are descended from osteoblasts that have been buried in bone matrix. They are not isolated and remain in contact with each other. They do this by gap junctioncoupled cell processes passing through the matrix via small channels, the canaliculi that connect the cell body-containing lacunae with each other and with the outside world. Osteocytes can sense pressures or cracks in the bone and help to direct where osteoclasts will dissolve the bone. They play an important role in initiating bone modeling or remodeling response based on mechanical load and strain changes and hence are important in mediating the effect of weight bearing physical activity on bone (Figure 6). 
Figure 6 : Types of Bone Cells [adapted from reference (24)]

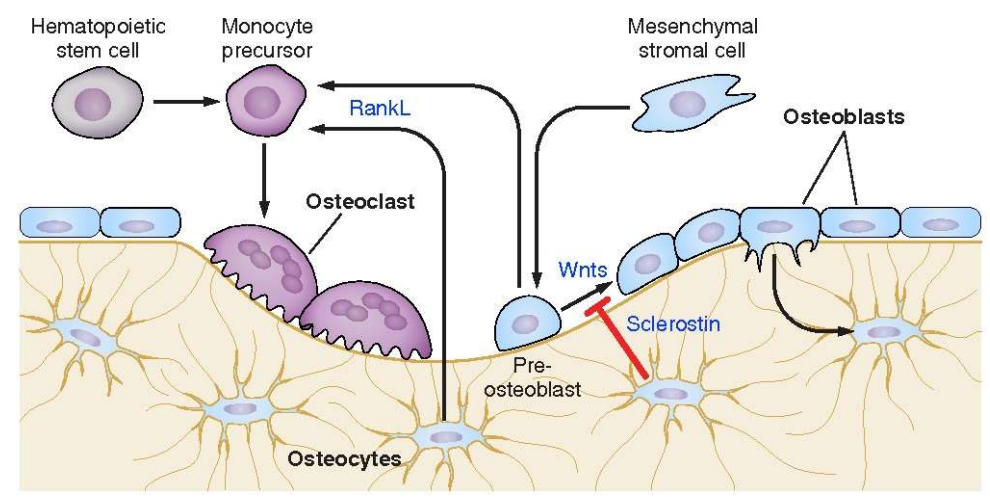

\subsubsection{Bone Remodeling Cycle}

Bone remodeling is an active process which involves removal of mineralized bone by osteoclastic resorption and deposition of new matrix by osteoblasts which subsequently mineralizes(25)(Figure 5). This process is critical for maintaining optimal bone health, and imbalance leads to net bone loss. Among the local factors that regulate bone remodeling the most important are RANK(Receptor Activator for Nuclear Factor-kB), RANKL(Receptor Activator for Nuclear Factor-KB Ligand) and OPG(Osteoprotegerin)(26). Cigarette smoking and excess alcohol consumption can affect the bone remodeling cycle.

Bone remodeling occurs in 5 phases (Figure 5).

\section{Activation Phase}

The first stage of bone remodeling is the activation phase, which initiates by detecting a remodeling signal. This signal can be either a mechanical strain on bone or a hormonal signal (estrogen or PTH). Daily activities can induce mechanical strain on the bone, which is sensed by osteocytes, who then translate this into biological signals to initiate 
bone remodeling. Under normal physiological conditions, osteocytes secrete TGF$\mathrm{B}($ Transforming Growth Factor Beta), which inhibits osteogenesis(25). Damage to the bone matrix and limb immobilization can damage osteocytes, increasing osteoclastogenesis by removing inhibitory signals. PTH (Parathyroid Hormone) hormone is an endocrine remodeling signal that is secreted by the parathyroid gland. It is involved in calcium homeostasis by acting peripherally on kidneys and bone and indirectly on intestine to maintain calcium levels. It acts via $\mathrm{G}$ protein coupled-receptor to activate osteoblasts through the intracellular signaling pathway to recruit osteoclasts to initiate bone resorption(27).

\section{Resorption Phase}

This phase involves osteoclasts digesting old bone. They do this through the RANK RANKL complex (25). RANK, a member of the TNF (Tumor Necrosis Factor) receptor family, is expressed on the cell membranes of pre-osteoclasts and mature osteoclasts (Figure 6). Its ligand, RANKL, is expressed by osteoblasts and marrow stromal cells. Any alteration of mechanical loading is sensed by osteocytes which release various factors in bone environment, recruiting the osteoclasts and leads to up regulation of RANKL receptors on quiescent osteoblasts. RANK stimulation by RANKL leads to activation of the transcription factor NF-KB, which drives the expression of genes that stimulate the functioning of osteoclast(25). Resorption is achieved by acidifying the bone matrix followed by release of lysosomal enzymes, such as CTSK (Cathepsin K), and MMP9 (Matrix Metallopeptidase 9), which degrade the organic component of bone(27).

\section{Reversal Phase}

The reverse cells (their full function is still unknown) perform this phase. These cells are mononuclear cell of undetermined lineage that help to clear the debris produced by 
bone degradation. There are many possibilities for what these cells might be but what is known is that within the bone they are likely to receive or produce coupling signals to link resorption with formation (27).

\section{Formation Phase}

This phase involves the osteoblasts which deposit new bone. Bone resorption leads to release of several growth factors like BMPs (Bone Morphogenetic Proteins), FGFs (Fibroblast Growth Factors) and TGF $\beta$, which are responsible for recruiting the osteoblasts in the reabsorbed area(25). Upon recruitment, osteoblasts produce the new bone matrix (osteoid) followed by mineralization. For the bone to assume its final form, inorganic hydroxyapatite is incorporated into this newly synthesized osteoid thus completing the bone remodeling process(27)(Figure 5).

\section{Termination Phase}

The termination signals that initiate this phase is largely unknown. Once mineralization is completed, mature osteoblasts undergo apoptosis revert back to a bone lining phenotype or become embedded in the mineralized matrix and differentiate into osteocytes. Resting bone environment in established until a new cycle of bone remodeling is initiated(27). 


\subsection{Peak Bone Mass}

\subsubsection{Definition of peak bone mass}

Peak bone mass is defined as the maximum bone mineral density acquired by the time a stable skeletal state has been attained plus subsequent consolidation which continues during young adulthood(28). Peak bone mass is acquired during childhood and adolescence, with peak trabecular BMD being achieved around 12-16 years of age (depending on gender) and peak cortical BMD being attained at around 20-24 years. Once attained, peak bone mass is relatively stable for a period of time before it starts to decline(Figure 7) with increased age and other associated factors which leads to increase risk of osteoporotic fractures(29). Peak bone mass is a major determinant of subsequent bone mass and osteoporotic fracture risk later in life $(4,30)$.

Figure 7: Peak Bone Mass Relation with Age[adapted from reference (31)]

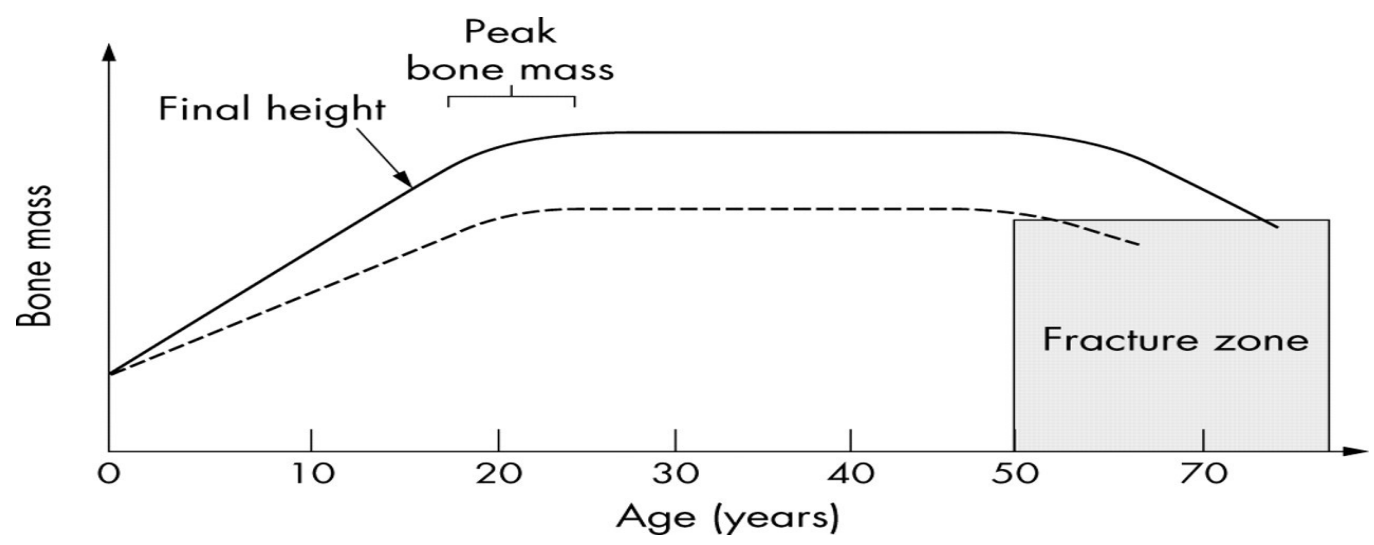

Some cross-sectional studies have suggested that in adult women, bone mass continues to increase during the third decade to reach a peak between 25 and 30 years(32). Much of the bone mass reaches a peak between the ages of 25 and 35 years(33). Although optimizing the genetic potential for peak bone mass is thought to delay the onset of 
osteoporosis and fractures in later life, longitudinal studies have yet to confirm this hypothesis. Men have bigger bones acquire greater bone mineral density and matrix deposition during the pubertal growth spurt and thus slightly higher peak bone $\operatorname{mass}(30)$.

\subsubsection{Skeletal Bone Growth}

As discussed above, we have a dense mineralized cortical bone and a spongy, less dense trabecular bone. Cortical bone has endosteum, which borders the marrow and periosteum, which is a fibrous layer with osteogenic potential that allows radial bone growth (also called as periosteal bone apposition). Longitudinal bone growth occurs at the growth plates through endochondral ossification (also called endocortical apposition) where pre-chondrocytes/resting cells first differentiate, proliferate, undergo hypertrophy, and subsequently mineralize(34). Cortical and trabecular bone differ in their responsiveness to disease effects, medications, muscle-loading and impact-loading physical activity, and hormonal changes. The process of bone modeling begins during fetal growth and continues up to the second decade of life(35). Bone modeling is sensitive to mechanical loading, emphasizing the importance of physical activity throughout growth (36). Skeletal characteristics such as cortical density and structural strength, that are determined by bone dimension and thickness, continue to increase into the third decade of life even after epiphyseal fusion, depending on the bone compartment and skeletal site under consideration(30) .

For an individual, peak bone mass is the maximum amount of bone accrued during adulthood which in turn depends on an individual's maximal or genetic potential for bone strength [i.e. BMC (Bone Mineral Content), $\mathrm{aBMD}$ (Areal Bone Mineral Density) and other measures of bone strength(30)]. The amount of bone mineral acquired from birth to adulthood follows age and sex specific patterns. Bone mass is acquired relatively 
slowly throughout childhood. With the onset of puberty and the adolescent growth spurt in height, bone mineral accretion is rapid, reaching a peak shortly after peak height gain (Figure 7). During the 4 years surrounding the peak in bone accretion, $39 \%$ of total body bone mineral is acquired; by 4 years following the peak, $95 \%$ of adult bone mass has been achieved(33).

There are several hormones and growth factors that regulate bone metabolism. These include:

1) PTH - This hormone regulates calcium and phosphate metabolism via its receptors (PTH1Rs) found on osteoblasts, osteocytes, cells of the intestine, and the kidney.

2) Calcitonin - a peptide secreted from the thyroid and neuro-endocrine cells that inhibits bone resorption.

3) Vitamin D - a secosterol hormone, which promotes calcium absorption by the intestine and decreases calcium and phosphorus excretion by the kidney.

4) FGF23 - a peptide secreted by osteoblasts, osteocytes and flat bone cells in response to high Vitamin D and phosphorous, increasing phosphorous excretion and maintaining phosphorous homeostasis.

\subsubsection{Importance of peak bone mass}

It is very important to optimize bone accrual during early adolescence as it is of great significance in preventing current or future fractures (Figure 8). Fractures in children or adults are associated with measures of bone mass, density and structural strength(12). 
Figure 8 : Bone development with Aging[adapted from reference(30)]

\section{Growth and Bone}

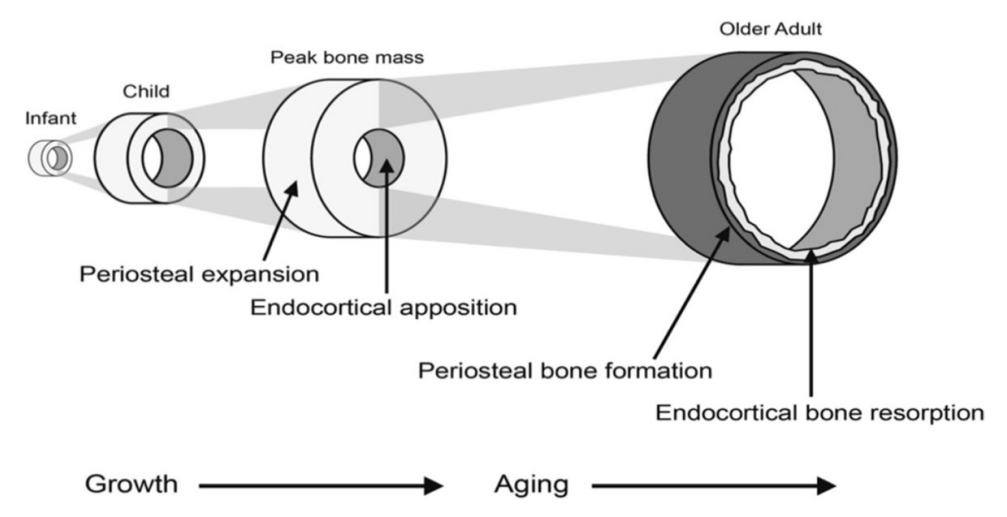

The frequency of fractures are higher among children compared to young and middle aged individuals(37), which reflects the vulnerability of the growing skeleton before attaining peak bone mass. A study showed early childhood fractures were associated with decreased bone mass gain in girls that could serve as markers for low peak bone mass and persistent bone fragility(38). This study was further validated by a recent Tromso study which found an association between the presence of childhood fractures and reduced bone mineral density among girls reporting low physical activity intensity. However a negative association between childhood fractures and bone mineral density was found in boys with vigorous physical activity and a history of forearm fractures(39).

Among healthy children, as many as one half of boys and one third of girls will sustain a fracture by age 18 years, with one fifth sustaining two or more long bone fractures(40, 41). One study also showed that there is a positive relationship between fracture frequency and level of physical activity due to an increased risk of falls during physical activity (42). This showed that although physical activity is critical for bone remodeling, it does not eliminate the risk of fracture due to higher levels of physical activity. On the other hand, stress fractures exhibit a different pattern from typical long bone fractures in adolescents. The lifetime prevalence of stress fracture among the general population 
is below $4 \%(43)$. Stress fractures are more common in women than men(44). White race and a reported family history of osteoporosis or osteopenia have been reported as significant risk factors for stress fractures, as have heavy physical activity and low vitamin D levels(45).

Understanding factors that affect bone strength early in life is important because low bone strength is associated with fracture risk in later life, independent of fall incidence and physical activity(46).

There are many factors that influence peak bone mass, which include genetic factors, hormonal factors, family history of chronic diseases, and environmental factors that include diet, physical activity and lifestyle choices. These factors are risks for developing osteoporosis and are discussed in greater detail in the next section.

\subsection{Risk factors for osteoporosis}

Osteoporosis is a multi-factorial disease with many clinical risk factors (Figure 9). We can broadly categorize them into non-modifiable and modifiable factors. This study is concerned with our knowledge of modifiable lifestyle factors beneficial to peak bone mass accrual, and specifically with the factors that help in preventing osteoporosis by ensuring those are optimal for bone health.

\subsubsection{Non-Modifiable Factors}

\subsubsection{Age}

Osteoporosis of later life is associated with aging and results from bone loss due to thinning of cortical and trabecular bone in both women and men(10). Once peak bone mass has been achieved, there is then a gradual loss in BMD as we age for the rest of our lives. In most populations hip fracture incidence increases exponentially with age(7). 
Increasing age is a risk factor for both men and women, but more for women. Above 50 years of age, there is a female to male incidence ratio of two is to one(12). Overall, around $98 \%$ of hip fractures occur among people aged 35 years or over, and $80 \%$ occur in women (because there are more elderly women than men).

\subsubsection{Sex}

As women age and undergo menopause, the drop-in estrogen significantly increases the risk of fracture in postmenopausal females, which is one reason why women are four to five times more prone to osteoporotic fractures compared to men. The United States Preventive Services Task Force (USPSTF) recommends the testing of all women aged 65 years and above and younger women whose fracture risk is equal to or greater than that of a 65-year-old white woman who exhibit no additional risk factors(47).

\subsubsection{Genetic Factors}

Genetic factors play a key role in skeletal mass accumulation and influence the pathogenesis underlying osteoporosis in both genders. Genes controlling this trait are yet to be fully determined. The familial resemblance of bone mineral content is expressed prior to puberty. Epidemiological and twin studies show less variance in BMD of monozygotic twins than dizygotic twin pairs $(48,49)$. We also know that ethnicity play a significant role in contributing to peak bone mass, with African Americans having highest BMD at all sites compared to other ethnicities, whereas Caucasians have a greater BMD compared to Asians and Hispanics(50).

Several candidate genes have been investigated. For example there have been recent reports examining the relationship between polymorphic alleles of the VDR(Vitamin D Receptor) gene and BMD with conflicting results(51). Even in studies where a significant correlation is demonstrable, the genotype-phenotype relationship was not uniform, with evidence for population, age, and site-specific variability. In addition, most of these 
studies have focused on postmenopausal populations, in whom interactions may be confounded by age and estrogen-related covariates. Genome-wide association studies(GWAS) have identified more than 70 loci associated with adult bone density or fractures(52).

A recent meta-analysis (53) and a more extensive literature review(54) support the initial hypothesis that allelic polymorphism in the VDR gene contributes to BMD, and that this genetic effect is more evident at the hip than the spine. A Canadian study by Rubin et al(55)did genotyping of the VDR and their findings confirm a significant association between VDR genotype and BMD at the proximal femur, but not the lumbar spine, even after adjusting for family history of osteoporosis, weight, age, and exercise.

\subsubsection{Sex hormone deficiency}

Estrogen and androgen are the two major sex steroid hormones in females and males respectively. These hormones are derived from cholesterol and synthesized in the gonads and adrenal glands. We know that sex steroids play an important role in bone homeostasis in both males and females(56). Although androgens are the main sex hormones in men, recent studies have showed that estrogen is the key regulator of bone health in both sexes(57).

Of all the hormones studied, estrogen is undoubtedly the most important hormone affecting bone metabolism and maintaining its structural integrity. In women, estrogen is made in the granulosa and theca cells of the ovarian follicles. Natural menopause occurs when ovaries decrease the production of estrogen. Sex hormones play a key role in acquisition of peak bone mass(58). The susceptibility of bone to sex hormone deprivation was documented more than 50 years ago by Albright(56), who noticed an association between osteoporosis and the onset of menopause. Since then, various studies have shown accelerated bone loss in women with estrogen deficiency, primary or secondary(58). While for men, the age-related decline in testosterone, adrenal androgens, growth hormone and IGF-1(Insulin-like Growth Factor 1) may contribute to 
reduced bone formation and bone loss, making them susceptible to fractures(59). Reduced BMD in men with fractures may be due to reduced peak bone size and mass, and bone loss. Bone loss occurs by reduced bone formation. This bone loss accelerates in elderly men because of endocortical resorption and increasing cortical porosity increase the surface available for resorption(59).

Recent studies have demonstrated that estrogen directly induces the apoptosis of osteoblast, thus reducing bone resorption and its protective effect on post-menopausal osteoporosis(60). Estrogen deficiency in postmenopausal women also leads to increase apoptosis of osteocytes, and the ability of bone to repair the micro damage depends on modulation of mechanical strain sensed by osteocytes and thus the viability of osteocytes in estrogen deficient women is associated with increased bone fragility(26, 61). Thus estrogen deficiency is associated with increased bone loss due to increased number of osteoclasts and osteoblasts, increasing the bone remodeling rate, which in unbalanced and leads to increase risk of osteoporotic fractures $(26,60,62)$.

In adulthood, most studies have reported that the OCPs(Oral Contraceptive Pills) had a neutral, or mild beneficial effect on bone health, because it usually contains estrogen along with progesterone(63). A case control study compared the BMD of premenopausal women who took the OCP with a control group and found there was no significant difference in BMD(64). Progesterone only OCPs may have a less favorable bone profile however(65).

Androgens are the major sex steroid hormones for men. However recent studies have suggested that a threshold level of estrogen in needed in men as well to prevent bone loss and with aging, an increasing number of men fall below this threshold, increasing their risk of fracture(57). In men, $15 \%$ of estrogen is synthesized in the testes and $85 \%$ comes from peripheral aromatization of androgen to estrogen. Androgen and estrogen both are important for developing peak bone mass and maintaining bone mineral density in men. 
Androgens are useful for maintaining cortical bone size by stimulating longitudinal and radial bone acquisition, whereas estrogen is found to maintain the cancellous bone by aromatization of androgen to estradiol. Studies have shown that estrogen played an important role in regulating bone resorption in men, whereas androgen and estrogen were both crucial for regulating bone formation(66).

Testosterone levels gradually decline with advancing age, but a large number of elderly men maintain their testosterone level well above the levels compared to that found in men with hypogonadism. Levels of SHBG increase with advancing age. This increase in SHBG further lowers the free testosterone and its aromatization to estrogen, thus declining both their levels(26) and rendering them more prone to osteoporotic fractures.

\subsubsection{Other Factors}

People who are born with genetic diseases such as Cystic Fibrosis, Gauchers disease, sickle cell disease, hemophilia to name a few are vulnerable to the development of osteoporosis(67). In addition, co-morbidities that often occur later in life such as Chronic Obstructive Pulmonary disease, end stage renal disease, diabetes mellitus, Cushing disease, congestive heart failure are also associated with an increased risk of fragility fracture. Other factors that also contribute to poor bone mineral density are glucocorticoid use for co morbid diseases such as inflammatory bowel disease, rheumatoid arthritis, athletics induced amenorrhea and anorexia nervosa(68). 
Figure 9 : Risk Factors for Osteoporosis[adapted from reference (29)]

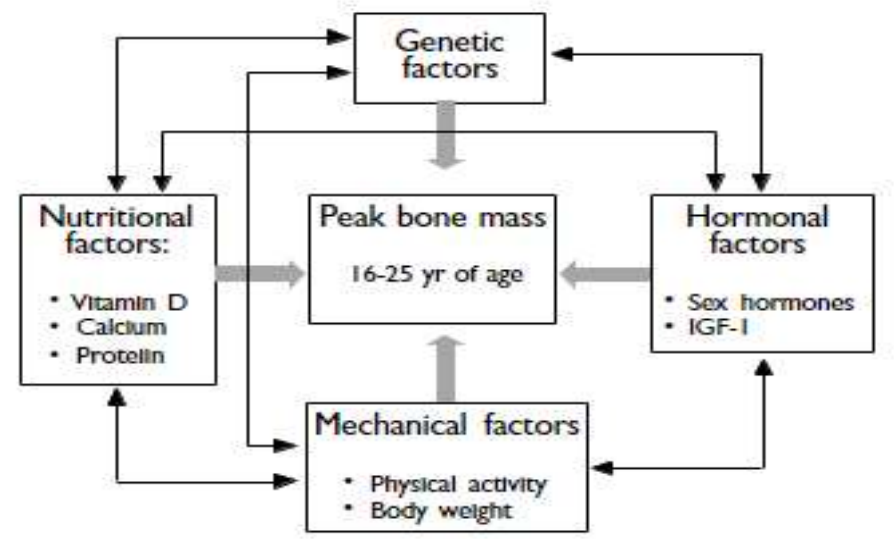

\subsubsection{Modifiable Risk Factors}

Environmental and lifestyle factors play an important role in bone health. As discussed above, peak bone mass is attained during the second and third decade of life(33), and the lifestyle adopted by adolescents plays a significant role in determining their risk of getting osteoporosis (Figure 9). These are the factors we wanted to explore among University students and is the crux of our study. More detailed discussion of these and their importance in adolescence and young adulthood is made below.

\subsubsection{Physical activity}

Growing bones are usually more responsive to mechanical loading than adult bones. The impact of physical activity appears to be stronger before pubertal maturation than during or after this period. Previous studies in young children, adolescents and young adults have reported that physical activity plays an important role in increasing bone mineral content, areal bone mineral density and cortical bone size(33). A recent Tromso study showed increased level of physical activity is associated with higher BMD and BMC 
levels in adolescents(69). For both sexes high activity frequency seems to be essential, whilst boys reporting quite hard intensity has an additional benefit(69).

Skeletal load that includes a dynamic load, a load with a high magnitude, a high frequency load, a fast load and a load with unusually distributed strains provide the most pronounced osteogenic stimuli. Weight bearing physical activity which generate high intensity loading forces augments bone mineral accumulation in children and adolescents(70). The amount of mechanical load necessary to stimulate osteogenesis is inversely proportional to the strain magnitude and frequency. The osteogenic response to high magnitude loading becomes saturated after a few loading cycles and additional loading thereafter has limited benefits. That is, the duration of exercise is of much less importance because a short duration of load or a small number of repetitions is enough to achieve the maximal anabolic effect $(71,72)$. Bone cell mechano-sensitivity seems to recover following a period without loading(73). Thus, separating loading into short durations with periods of rest optimizes the osteogenic response to loading, even to low magnitude stimuli $(74,75)$.

It has been found that adults who maintain high levels of weight bearing exercise in their first three decades of life maintained their peak bone mass, thus attenuating bone loss associated with aging(76). Gravity bearing exercises(like jogging, running), activities that involve jumping (volleyball, basketball), and resistance exercises(weight lifting) have shown to have positive impact on the peak bone mass by stimulating to increase osteoblasts activity(77). This increases cortical thickness acquired during the second decade of life(33). Physical activity has been suggested an a possible therapy to prevent osteoporosis; in one recent study Ho-pham et al showed that physical activity is an important intervention for prevention of bone loss and osteoporosis in adults(78). 


\subsubsection{Body Composition}

It is widely recognized that lean body mass is one of the strongest correlates of bone mass, density, and structural strength during childhood(79) although some recent studies also highlight the importance of fat mass. In the latter phase of the adolescent growth spurt, following the peak, continued gains in lean mass are strong predictors of increases in BMC (80). While one recent study demonstrated that high BMI was protective against femoral neck osteoporosis(81), the Tromso study found that both lean mass (LM) and fat mass (FM) should be regarded as strong predictors for bone mass and bone strength in adolescents, with gender specific differences. In boys high LM was important for bone strength whereas for girls with low LM, high FM seemed to ameliorate the effect of deficient LM(82).

\subsubsection{Dietary supplementation}

Dietary intake of foods rich in calcium helps build strong bones in children and adolescents. Calcium is mainly found in dairy products, as well as in green leafy vegetables, legumes, nuts and certain types of fish. The bioavailability of calcium in green leafy vegetables is generally high, but the quantity of vegetables needed to be consumed in order to meet requirements is quite large, giving dairy the advantage as a primary source of calcium. The American Academy of Pediatrics recommends to periodically access calcium intake in growing children and adolescents and encourage increased intake of either dairy or calcium rich foods(83).

A previous study found that calcium supplementation, whether with pills, fortified foods, or dairy, consistently increases gain in skeletal mass and density measures in children and adolescents, usually between 1.0 and 5.0\%(30). Calcium supplements are another option in order to meet their body requirement of calcium. However, a recent meta-analysis showed that routine supplementation of calcium supplements only leads 
to a very small increase in BMD, which does not reduce the risk of fracture either in childhood or later in life(84). However, while low dietary intake of calcium may be associated with an increased risk of fracture(84) there have been few ecological studies conducted where low calcium intake was associated with low levels of fractures in older population(85). This finding seemed to be related to high level of physical activity in Japanese women in their young age which could have led to greater acquisition of peak bone mass(85).

Per capita consumption of dairy in New Zealand for the year 2018 stood at $105.26 \mathrm{~kg}$ which is higher than Australia, Canada and United States (CLAL.it). However, despite the statistics stated above, significant numbers of New Zealand adolescents avoid dairy for environmental reasons, leaving them to look for alternative calcium sources in their diet.

Vitamin $D$ is another essential nutrient important for bone health. Chronic and severe vitamin $\mathrm{D}$ deficiency leads to osteomalacia, a metabolic disease characterized by low mineralization of bone. The major causes of vitamin D deficiency and insufficiency are decreased renal hydroxylation of vitamin $D$, poor nutrition, scarce exposition to sunlight and a decline in the synthesis of vitamin $D$ in the skin. There are a number of studies on the effects of vitamin D supplementation on bone loss in the elderly, showing that supplementation with daily doses of $400-800$ IU of vitamin D, given alone or in combination with calcium, are able to reverse vitamin $D$ insufficiency, to prevent bone loss and to improve bone density in the elderly(86). Adolescents are a population group at high risk of low vitamin D status, which is concerning given the important role of vitamin $\mathrm{D}$, and calcium, in promoting normal bone mineralization and attainment of peak bone mass during this rapid growth phase(87). A recent evidence based study indicates that vitamin $D$ intakes of between 10 and about $30 \mu \mathrm{g} / \mathrm{d}$ may be required to avoid deficiency and ensure adequacy in adolescents, considerably greater than the current recommendations of $10-15 \mu \mathrm{g} / \mathrm{d}(87)$ while in the elderly, the SENECA study 
evidence supports routine supplementation of 700-800 mg of calcium and $400-800 \mathrm{IU}$ of vitamin $D$, which is an effective, safe and cheap means of preventing osteoporotic fractures(86).

\subsubsection{Alcohol}

Alcohol consumption during the early adolescent years decreases peak bone mass and may result in relatively weak adult bones that are more susceptible to fracture(88). A cohort study performed in Portugal in young adolescent girls found a negative association of alcohol intake with $\mathrm{BMD}(89)$, while a similar study conducted in the Cincinnati Children's hospital in young adolescent females found that there was no association between alcohol and $\mathrm{BMD}(90)$, whereas in boys, mild to moderate alcohol consumption was significantly and positively associated to BMD at the hip sites. This was supported by findings in adults where moderate alcohol consumption appears to be beneficial to men's bone health(91). The mechanism behind this has been suggested to be promotion of endogenous estrogen synthesis, thereby increasing BMD in individuals with low circulating levels of estrogen(91). These studies suggest that adolescents' and adults should limit their alcohol consumption to moderate quantities and not indulge in binge drinking which can have adverse effects on bone health.

Long term heavy alcohol consumption can interfere with bone remodeling directly or indirectly thus decreasing bone density. There is a dose-dependent relationship between alcohol intake and fracture risk. Daily intake of 3 or more units of alcohol is associated with a higher fracture risk (88). Schnitzler and Solomon (92) found that alcohol administration reduces bone formation and increases bone resorption. A microscopic analysis of bone tissue from men with osteoporosis confirmed that heavy alcohol consumption leads to delayed and impaired osteoblast activity associated with normal osteoclast function. This imbalance results in part from alcohol induced 
inhibition of osteoblast, specialized cells that deposit new bone, thus leading to decreased bone mass(93).

There have been other studies which show a correlation between alcohol consumption and its effect on bone health. However very few studies have been conducted in young adolescents aged below 15 years.

A recent study by LaBrie et all at Loyola Marymount University, Los Angeles, California (2018) showed an association HED(Heavy Episode Drinking) and poor bone health in adolescent young woman(94). Frequent HED(having four or more drinks within 2 hours on 115 or more occasions since the start of high school, which is approximately equal to 1.6 episodes per month over this period) was associated with decreased vertebral BMD even when variables most commonly associated with bone health(lean body mass, physical activity, age at menarche, smoking, and oral contraception use) were controlled for. However, early HED initiation (beginning HED at age 15 years or younger) was not significantly related to BMD. Another recent study(95) that was actually undertaken at Victoria University considered lifestyle factors for fracture that were present in groups of alcohol dependant, premenopausal women and found that women being treated for alcohol dependence also commonly reported cigarette smoking, low dietary calcium intake, previous fracture, and high incidence of falling (95).

\subsubsection{Smoking}

Most epidemiologic studies have found that BMD in older male and postmenopausal female smokers is significantly lower than that in nonsmokers, even after the effects of age, body mass index, and other lifestyle factors have been accounted for. However far fewer data exist in younger adults. A meta-analysis was conducted by Kanis et al to consider this issue, and showed that for any osteoporotic fracture, the risk ratio increased with age, but decreased with age for hip fracture. A smoking history was 
associated with a significantly increased risk of fracture compared with individuals with no smoking history, but the risk ratios were lower than for current smoking(96). The Tromso study looked into lifestyle factors that have effect on BMD in young adolescents and found that smoking was negatively associated with BMD at the hip only in boys but not in girls(97). This difference was most likely attributed to the fact that girls were less likely to smoke.

The mechanism by which smoking affects bone health, the breakdown and hydroxylation of estrogen by the liver is increased in smokers. Less circulating estrogen implies less protection of bone mineral density. It is postulated that smoking also inhibits the aromatization system, thereby further reducing estrogen.

In men, the effect of smoking on testosterone levels in unclear. In most recent observational studies, it is primarily the level of free serum estrogens rather than free testosterone that predicts BMD in older men. The inhibition of aromatization of androgens to estrogens explains the increased levels of testosterone and androstenedione found in both male and female smokers.

Smoking rates have drastically declined in New Zealand from 2006 to 2018 . There has been a decline of $4 \%$ in adult smokers compared to youth, where the margin of decline was significant reaching up to $12 \%$. Maori population had a decline of $7 \%$ while the pacific adults had decline of $2 \%$ (Figure 10). 
Figure 10: New Zealand's Smoking Rates (Ministry of Health. New Zealand Health Survey $(2006 / 07-2017 / 18)$

\begin{tabular}{|c|c|}
\hline Demographic & Percentage \\
\hline Adult smokers (15+) & $\begin{array}{l}16 \% \text { (down from } 20 \% \text { in } 2006 / 07 \text { ) } \\
\text { - With higher smoking rates among men (17\%) } \\
\text { than women ( } 14 \%)\end{array}$ \\
\hline Youth aged $15-17$ & $4 \%$ (down from $16 \%$ in $2006 / 07$ ) \\
\hline Young adults $18-24$ & $20 \%$ (down from $28 \%$ in $2006 / 07$ ) \\
\hline Māori adults & $\mathbf{3 5 \%}(42 \%$ in $2006 / 07)$ \\
\hline Pacific adults & $\mathbf{2 5} \%(27 \%$ in $2006 / 07)$ \\
\hline European and other & $14 \%$ \\
\hline Asian adults & $8 \%$ \\
\hline
\end{tabular}

\subsubsection{Other Factors}

Some of the other factors that are modifiable are sex hormone deficiency among young men with hypogonadism and low testosterone levels. These young men were at risk for osteoporotic fracture as they were found to have low bone density(98). Therefore, such individuals may benefit with testosterone replacement therapy in contrast to administering testosterone to elderly men, which may cause undesirable side effects related to increased prostate size and may even promote the development of occult prostate cancer. Long term immobilization and high salt intake are some other modifiable factors that affect bone mineral density(67). With appropriate supplementation of vitamin D, eating diet rich in calcium and decreasing salt intake can lead to increase in BMD and reducing the risk for fracture(67) . 


\subsubsection{Summary}

Osteoporosis is a major public health problem through its association with fragility fracture. Peak bone mass is attained in the second or third decade and has been shown to be a major determinant of later osteoporosis risk. Important determinants of peak bone mass include physical activity levels, diet, cigarette smoking, and alcohol consumption. This study aimed to identify lifestyle barriers for attainment of optimal peak bone mass and to elucidate knowledge of factors affecting bone health among young people through focus groups of young adults recruited at Victoria University of Wellington. 


\section{Chapter 2: Methods}

\subsection{Overview}

This research was conducted at Victoria University of Wellington, New Zealand. Six semi structured focus group interviews were conducted between August 2018 and February 2019. The interviews were held with twenty-five students and three staff members from Victoria University of Wellington, New Zealand, aged between 18-35 years of age.

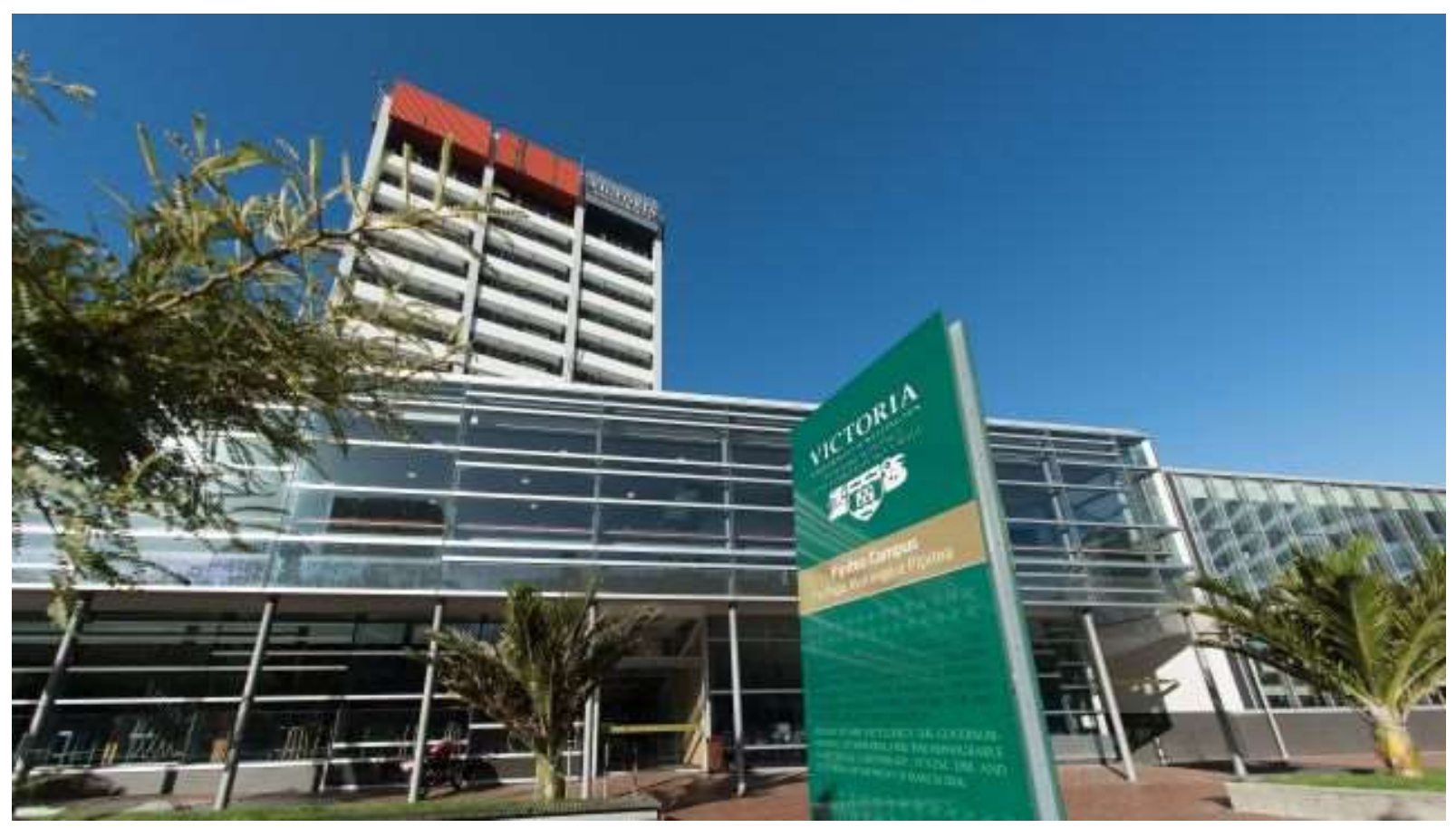

Participants were recruited via flyers on campus, internal emails invites and through personal interaction on campus. The study was open to anybody who was between the age group 16-35 years of age. Participants gave written informed consent to be a part of this study. 
Detailed focus groups were held in quiet, private meeting rooms at the university campus. The first two focus group interviews were conducted by the primary investigator (SZ), a secondary investigator (HP) and co-supervisor (HD). The next two focus group interviews were conducted by the primary investigator (SZ), co-supervisor (DH) and supervisor (ED). The last two interviews were conducted by the primary investigator alone.

Before attending the focus groups, participants were emailed study information sheets, which provided a background about the study, methods and confidentiality aspects pertaining to the study (see appendix). On the day of interview, written consent was obtained from each participant. A \$20 gift voucher card was given to each participant as an expression of thanks at the end of the focus group.

We used 6 semi structured focus group interviews as the tool for data collection for this study. A set of structured questions were prepared beforehand, which was then directed towards a particular subject of interest as the interview progressed (see appendix). The interview started with a basic open-ended question "What do you understand about Bone Health"? Leads were then taken further to assess the understanding of participants about peak bone mass and the barriers they face in acquiring an optimal peak bone mass.

Each focus group was audio taped electronically using an IPad and an audio recorder. Each interview was transcribed by the primary investigator (SZ) to produce a verbatim textual file. The data collected was analyzed thematically by the investigator using Nvivo software (Version 12). Relevant data fragments were coded, and a constant comparative approach was used to ensure the codes were used consistently. These codes were grouped into categories and summed into main themes. The investigator (SZ) then analyzed these final emerging themes, which were assessed against the coded transcripts by the investigator (SZ) and sent back and forth to the co-supervisor (HD) and supervisor (ED) to ensure they represented the data appropriately. The constant 
review by the co-supervisor (HD) and supervisor (ED) ensured that the integrity of the data and the results were maintained.

Focus groups were conducted until the same themes and sub themes kept appearing to the point where we did not hear any new theme or subthemes i.e. data saturation was reached. One more focus group was conducted at this point as is usual for qualitative research.

\subsection{Research Goals}

The aim of this thesis was to look into attitudes and knowledge in young adolescents towards attaining an optimal peak bone mass and risk of osteoporotic fractures. We were also interested to investigate the barriers that young people face in adopting a lifestyle associated with optimal peak bone mass acquisition.

\subsection{Research Questions}

The aim of the study was to answer the following two main research questions, which were:

1) What do adolescents and young adults understand about peak bone mass and risk of osteoporotic fracture?

2) What are the barriers that young people feel in adopting lifestyles associated with better bone health and how should we reduce this? 


\subsection{Research Methodology}

A focus group methodology was chosen for this research for the following reasons:

1) The focus group methodology can be used for idea generation. It gives insight to people's thinking, their opinions and their feelings as to how they perceive this particular topic. It can give rise to flight of ideas, which can progress in a variety of directions pertaining to the topic.

2) The casual environment with the researcher helps participants feel at ease and less judged, thus allowing themselves to express their opinion freely.

3) The research question selected was not a sensitive topic, thus allowing participants to discuss their own lifestyle experiences.

4) A focus group can gather a large amount of data in short period of time. Thus, they are appropriate for collecting in-depth information on a topic with a limited time frame for data collection(99).

\subsection{Ethical Considerations}

Ethics approval for this study was granted by the Health and Disability Ethics Committee (Ref: 18/CEN/18) and Victoria University of Wellington Human Ethics Committee (Ref: \#023752). Each participant was emailed the study information sheet and consent form prior coming to the interview. On the day of focus group, written consent was taken on hard copies from the participants at the beginning of the interviews. Participants' consent was sought for the focus groups to be audio taped. Participants could choose a pseudonym to put on their name tag, thus enabling the discussion to flow freely without interrupting to ask for names. It was also explained that they could leave the discussion any time they wished without providing any reason. 


\subsection{Participants}

The targeted population was students and staff from Victoria University of Wellington. Posters and flyers were put on notice boards in the University campus with tear off slips containing the investigator email address and contact number. Mass mailing was also used to recruit participants. Interested participants contacted the researcher to enroll in the study. There were no specific inclusion criteria except an age group of 16-35 years. Focus groups were conducted on campus to make it more accessible to participants. Quiet and comfortable meeting rooms were chosen with a center table and chairs surrounding it, thus allowing the participants and researcher to face each other and interact comfortably. Fresh fruit and chocolates were provided as refreshments along with water and juice.

A total of 6 focus groups were conducted over 6 months. The number of focus groups was determined by data saturation. The first two focus groups were conducted in August 2018. These were transcribed verbatim by the primary researcher. The next two focus group were conducted in December 2018, followed by transcription. And the last two were performed in February 2019. Once data saturation was achieved, a collective decision was taken by the researcher and her supervisors to stop conducting focus groups.

A total of 28 participants participated in these focus groups: 7 males and 21 females aging between $16-35$ years participated. The mean age of participants was 28 years. The minimum number we had in a focus group was 2 participants while the maximum we had was 11. Although each focus group had 8-12 participants invited, on some days the participants did not show up as anticipated, thus decreasing our size of the focus group. But each focus group had around a mean of 5 participants, in line within the recommended group size(100). 


\subsection{Data Collection}

After the participants confirmed their availability, emails confirming the time, date and venue were sent out. A reminder email was sent a day before to all the participants.

The participants were encouraged to talk to each other and discuss ideas. Each focus group lasted around 1 hour. At the end of interviews, the summary was summed up to participants followed by a question and answer session. This session lasted a couple of minutes with the researcher answering questions and educating the participants about better ways to improve bone health. At the end of which, every participant was given a grocery voucher as a token of appreciation for their participation.

\subsection{Guide Questions}

A set of semi structured guide questions were used to steer the participants towards answering our two main research questions. The interview started with the basic question: "What do you understand about bone health?". This brought out quite a lot of opinions which were then directed more specifically to the barriers and enablers faced by young adults in day to day life.

The guide questions asked were the following:

\section{Questions About Knowledge}

1. What do you understand about bone health?

2. What factors do you think have negative or positive impact on your bone health?

3. What kind of food do you think effects bone health?

4. What about smoking?

5. Would smoking affect bone health?

6. What about alcohol consumption?

7. Would alcohol affect bone health?

8. Have you ever heard about Osteoporosis?

9. Do you think there is enough awareness/knowledge about attaining better bone health? 


\section{Questions About Behavior}

1. What are the lifestyle factors you would choose to keep your bones healthy and strong?

2. What changes did you bring in your lifestyle with the knowledge you have about bone health?

3. How important are physical activities in your day to day life?

4. What prompts you to stay physically fit?

5. What changes did you bring in your diet to have strong bones?

6. What made you start smoking? What kept you going?

7. Was there anything that stopped you from smoking?

8. How happy are you with your alcohol intake?

9. Have you ever sought any medical care for bone pain/fracture?

\section{Closing Questions}

1. How do you think your bone health relates to risk of fracture later in life?

2. If you had to sum up the barriers you face daily to attain a better peak bone mass, what would they be?

3. How would you overcome those barriers?

\subsection{Transcription}

The audio taped files of focus group interviews were transcribed by the lead researcher through manual process of listening and writing the verbatim text on Microsoft word file.

\subsection{Analysis}

The data that was collected was analyzed using a constant comparative methodology. The transcripts were read back and forth, and emerging themes were coded using the Nvivo software. These codes were grouped into themes which gave us our results.

Initially, each verbatim was read and important data was coded as nodes in Nvivo. The same process was applied until the investigator had been through all the verbatim 
coding them into important pieces of information. After doing so, the investigator went back and reorganized the codes, grouping them into themes. The emerging data was shared with the supervisors at all stages to make sure the codes were tested and analyzed using constant comparative methods. 


\section{Chapter 3: Results}

\subsection{Overview}

From the data, around 15 themes emerged that answered the following research questions. Seven themes were identified that answered our research question 1. And broadly eight themes were identified which answered our research question 2.

1) What do adolescents and young adults understand about peak bone mass and risk of osteoporotic fracture? (Table A1)

2) What are the barriers that young people experience in adopting lifestyles associated with better bone health and how could we reduce these? (Table A2) 


\subsection{A1 Research Question I}

The following seven themes were generated pertaining to research question 1.

\section{Table A1: Knowledge}

Theme

1. Physical Activity

2. Dairy \& Supplements

3. Alcohol

4. Smoking

5. Lack of Knowledge of sequelae of poor bone health

6. Menopause

7. Aging \& Medical Illness
Sub-Theme

I Exercise

II Resistance Training

III Gym/High impact

IV Overuse

I Dairy intake

II Calcium \& Vitamin D

III Diet/Nutrition

IV Alternative for Vegans

I Cultural Factors

I Bad for Health

I Osteoporotic Fractures

I Estrogen Deficient

I Risk for fracture

\section{Summary}

Bone health was a subject that many participants felt they had little knowledge of. Most of them related bone health to the amount of calcium one gets from food sources or from dairy intake. One of the most discussed themes was exercise; it emerged in every discussion with people acknowledging that it has positive effects on overall health and not just bone health. It was something which everybody tried to incorporate in their day 
to day life. Of the factors that were deterrent to health, many participants admitted that social habits like smoking and alcohol seem to have an adverse effect on overall health and not just bone health particularly. Participants further divulged details about how peer pressure during social events had a positive or negative impact on their level of alcohol consumption and smoking. Lastly participants concurred that bone health deteriorates as we grow old and attributed increasing age as a risk factor for fractures later in life. While most of them had heard about osteoporosis, many agreed they did not know what factors would increase their risk of osteoporotic fractures later in life. There was scant knowledge about it and the changes one can bring in their life to decrease their risk of osteoporosis.

The themes are discussed in detail below. To maintain the confidentiality of the participants their real names have been replaced with pseudonyms.

\subsubsection{Physical Activity}

A major theme that emerged in the focus groups was physical activity. This was discussed in depth in almost all focus groups. The majority of participants did some sort of physical activity every day to keep themselves overall healthy and fit. However, bone health consideration mattered to very few of them. Among the participants, very few of them held the knowledge that weight bearing/resistance training had positive effects on bone mineral density. The ones who were aware of this knowledge were fitness enthusiasts while the rest of them were not aware about the beneficial effects of weight bearing exercise on peak bone mass. For most of the participants, incorporating physical activity in their daily routine was to stay fit and healthy. 
As mentioned by Jake,

"If someone asks me what do you think the aspects controlling, that would improve your bone health, you would probably go through and say exercise or physical activity, I am sure, helps in some way. There's not many things that physical activity doesn't help in some form, so I guess just as $a, I$ do it as an allencompassing......, just trying to keep my body in a good place."

On further exploring this factor, participants were asked what really motivated them to keep doing exercise, and for most of them it was overall fitness rather than just bone health as a reason to workout. A lot of participants showed awareness and knowledge about exercise and its beneficial effect on health and demonstrated the will to incorporate some form of exercise in their day to day life.

Another interesting reason with regards to knowledge about bone health was that poor bone health doesn't present with symptoms early in life. Due to this a lot of them never focused on bone health but focused on overall fitness. As quoted by Olivia, who never thought about bones while growing up.

"I am not really thinking about bone health, you know when I choose the type of activity I am doing but also when I try to motivate myself to go I don't think 'I am going to work out 'cos then I'll have healthy bones. That's one of those things that makes sense but like what you were saying [to another focus group participant], it's sort of a general health benefit, I don't think about the specific aspects of it. And also, I have never really fractured a bone except my tail bone so it's not something I have had to worry about. I feel like maybe if I broke a bone as a kid, I might be more inclined to think well how can I prevent that from happening in the future. It wasn't really a type of thinking that I have developed growing up."

When exploring what kind of physical activity had an impact on bone health, we had some interesting responses from fitness enthusiasts. Fitness enthusiast were the 
participants who engaged in physical sports 5-7 days a week. These participants were aware of the benefits of resistance training and tried incorporating it in their day to day life in some way. They also displayed knowledge about how doing high impact physical activities can do more harm than good. Participants talked about why they preferred such exercises in their daily life with most of the time the motivation being fitness. However, this was only a small number of participants who had this knowledge and represented a very small section of the focus groups. As Sharon said,

"Well I guess for me from experience is that too much high impact activity can be actually detrimental, can really wear down your cartilage and your bones, and also especially for growing, it's not great to do that. Yeah I think that's one of the reasons why I went to swimming because you are still kind of gain some resistance training."

But for most of the participants the reason and motive to stay physically fit was the health benefits associated with exercise such as better mood, more energy levels, better concentration and focus at work and overall fitness. Quite a few participants also reported the detriment in their health if they stopped any kind of physical activity. Moreover, working out every day made a lot of participants feel more productive every day and that was a major incentive to some. As said by Lisa,

"On one side it keeps me focused. Because, I have my days planned out, I have my gym work out planned out. And just sticking to it, I feel like I am already very productive throughout the day. I know exactly what's coming next and you know it's just making me feel really organized and gives me this feeling of achievement. But also it's a way of, you know, that's where I socialize with some friends, where I can de stress from the Uni stress. It's just something I really enjoy, it's like this little break that I get from my really stressful academic life, or work life and you know the side benefit is that makes my body better, I hope." 
Andrew said something similar too,

"Cause when I stop doing, I feel you know, I start feeling like very low energy, bad mood, I don't sleep well, I am not productive, you know everything goes stop. But that time, you feel you have to go back on track and do some more exercise, cause you kind of feel the benefits and everything kind of gets better, everything in your life - you are in a better mood, start the day with more energy, you know."

For some participants, excessive exercise in the past had resulted in sports related injury, which then led them to take their bone health more seriously. Moana has had such an experience,

"I'd get injured every year. It started off with broken bones, like broken ankles, twisted wrists or whatever, and then in my last year of high school when I ruptured my Achilles two times, that's when they were like 'ooh stop', like instead of going rehab for like 3 months, you need to go into rehab for about 3 years so yeah. So I've had to really think about my bones, but I was sort of like you [to another focus group participant] before, when I was playing when I was young, I didn't even think about it, I just played sport because I loved it and it was fun and I got to, you know, socialize with my feelings but now it's like, this affects the way I walk, it affects the way I get to places so I need to actually think a bit more realistically like not so much the social aspect but more practical."

Another important aspect of being able to go to the gym everyday was peer pressure. Participants were more likely to go to the gym more often and consistently if they had friends at the gym or friends going along with them to the same gym every day. This increased consistency among participants, and they were more likely to stick with their routine of exercise every day. 
Quoting Caleb from excerpt,

"Yeah, it's definitely a pull factor for me. If a friend wants to go to the gym or whatever, I am more likely to go with him than I would by myself. And then if you get into a regular routine of doing that, you know, each week, I am more likely to go, continue going, even by myself. Because sometimes you are not motivated to go by yourself, just I want to sleep in."

To summarize, most people were well aware of the health benefits of exercise in their daily life. Most of them had some form of physical activity in their day to day life, while for a few it was a constant struggle to do so. Participants displayed a strong will in maintaining physical fitness, be it for self-care or for fun. Overall, despite doing so, there was very little knowledge on how physical activity had a positive impact on peak bone mass and that if worked upon, could bring significant changes in one's bone health. This theme also demonstrated the lack of awareness about different benefits of exercise, especially for bone health.

\subsubsection{Supplements \& Dairy}

Almost everybody in the focus groups had the opinion that calcium intake is probably good for bone health. This was the first factor everybody talked about when asked "what factors do you think affect bone health"? Almost all the participants concurred that they grew up learning calcium is good for bones and most of our dietary calcium intake is from dairy. This is what most of the participants associated with better bone health. As Andrew said,

"I guess what they told you when you are growing up like, you have to drink milk because of the calcium that makes your bones strong. And, dairy products, I mean you associate dairy products to healthy bones." 
We had a few participants who made sure dairy was part of their daily life to keep up with their calcium supply and it was mostly this that they had learned growing up. Like Zuha said,

"Ummm yeah so I drink a glass of milk every day and I like cream and cheese stuff as well."

On the other hand, there were participants who were not as mindful of how much of calcium they consumed on a day to day basis but agreed that dairy was the only source of calcium they knew of when it came to bone health. For participants who were vegan, and dairy was not an option, a few of them supplemented themselves with calcium supplements and foods that were rich in calcium to meet their daily calcium requirement. As Kate mentioned,

"I don't really drink a lot of milk or dairy. So, I have in the past, had some of those, the calcium chews that are like really yummy but yeah, I am also just trying to eat lot of broccoli, apparently that's good for calcium."

Quite interestingly we also had few participants commenting on Vitamin $D$ being important for bone health. We had some participants who had very good knowledge about vitamin D. They commented on how staying in sunlight helps increase the levels of vitamin $D$ in our body, which was a good level of knowledge resource but was found to be in very few participants. Quoting Sharon,

"I was just thinking another thing was as well that you are living in apartment and you are also inside all day that you can't really get out into the sun as much, then you don't get as much vitamin D."

But there were also two participants who were taking Vitamin D supplements on prescription for other related medical illness and were not quite aware of its benefit on bone health. 
As Olivia mentioned,

"I started taking Vitamin D because it's supposed to help with Asthma. But I feel like on the bottle it says something about bone health (laughing). I haven't actually read the bottle. I went to my GP and said I was having problems controlling my asthma and he suggested Vitamin D and I just take Vitamin D now."

We also had participants, who had good in-depth knowledge about vitamin D and how it helps with absorption of calcium. But this was due to their own medical illness which led to this knowledge. As Sara said,

"I take prescription of Vitamin D.... My doctor told me to take it. Because I have Crohn's disease, so I probably don't get enough calcium that goes in my diet so for absorption I have to take it."

Most of the participants who were vegan and wanted to stay healthy and eat nutritious food that is rich in calcium faced difficulty because they admitted they lacked knowledge on alternative sources of calcium apart from dairy. As Sharon said,

"I think we shouldn't just be getting dairy. I think there should be other information sources about, you know other things that we can be eating or supplementing with."

Television and social media had big role to play here, with many admitting this was a major source of information relating to health. However, quite a few felt that information may be biased and commercially driven with very little information available on alternative healthy food alternatives for calcium. As Sharon commented,

"I feel like that's the only source where you are kind of getting told that you can only get calcium from milk products. You know, it's in your face, Fonterra group is putting it out there. Um yeah, that's almost like the only information source out there that's on the TV, on the billboards, it's always for dairy. " 
This theme was certainly one of the most talked about, with most of the participants in every focus group commenting on their knowledge about dairy intake being associated with better bone health. However, in addition to intake of dairy, other factors acted as a barrier in attaining better peak bone mass. The barriers are discussed in next section.

\subsubsection{Alcohol}

The next theme that emerged in regard to knowledge about the factors that affect bone health was alcohol. Alcohol has long been associated with many disease risks by having detrimental effects on health. Instead of bone health concerns, most of the participants saw its consumption to be detrimental to overall health. On being asked if she ever thought about alcohol affecting her bones, Sharon had to say this,

"Not so much about the bones but more just about affecting my brain health and yeah stability from there, so yeah just overall long terms affects really"

A lot of participants did display knowledge that alcohol can have a deleterious effect on the liver, which is why it should be consumed in moderation. Not doing so, could lead to a wide array of health problems. As Anna aptly said,

"Yeah, I mean cos like if let's say you consume, you consume too much alcohol, obviously your liver and like your kidneys like will get messed up. So that might affect the rest of your health and you know you might die fast."

Although many agreed that excess alcohol consumption was bad for one's health, participants did not hold any knowledge of how it affected peak bone mass or bone health per se. It wasn't something many paid attention to. 
As Olivia commented,

"Yeah, I don't really have thought about it actually. I haven't really, I am like, I am not aware of there being effect of alcohol on bone health. It's definitely not, it registers at all when I am thinking about whether I should drink. I mean it's one of those things where I know alcohol is bad for your health, so I'd be surprised to learn that it doesn't have a negative impact but I am certainly not aware of it or actually ever thought about it specifically."

A large number of participants avoided alcohol because they had knowledge regarding its deleterious effect on health and also the immediate side effects one experiences after consuming alcohol. This seemed to be a major turn off for such group of participants. Like Moana said,

"Yeah, personally I don't drink so either. It's because I sort of think forward, so like I don't want to wake up the next day, not knowing where I am and like being so worried about what I have done to my body. But because you live in a culture where you don't think of that and you have alcohol in a positive manner. That's what I think it is."

Another deterrent in alcohol drinking was its cost where some found it too expensive and so avoided it for that reason. Lastly, although drinking alcohol came with lot of side effects and negative health consequences, there was a positive side to it as well. People attributed alcohol related events for finding who their true friends are and who supported their decision to drink or not. As Sharon said,

"....... it's a good thing cause It helped me decide like who my real friends are, you know like, who's willing to hang out with me without alcohol and who really wants to hang out with me when I am drinking so, yeah. There's a positive out of it, you know who your real friends are. " 
To conclude this theme, alcohol was recognized as a deterrent to one's overall health. Its consumption was often linked to more of social obligation than the interest in alcohol itself. For the people who avoided it, it was mostly health reasons or due to its side effects. However, not many were aware of the fact that alcohol consumption had an effect on peak bone mass.

\subsubsection{Smoking}

Another theme that emerged in all focus groups was smoking. Almost everybody in the focus groups agreed smoking tobacco cigarettes is bad for health and there is no benefit from doing it. But like alcohol, not many were aware about the effects of smoking on bone health. Participants agreed they never thought about the effect of smoking on bones and most of them associated smoking with lung problems. On asking participants if they knew what impact did smoking have on bone health, we had the following responses. As Jake said,

"I was just thinking. I have no idea. Like its one of the things where if someone asks me do you think that exact question, yeah well maybe, you classically think of lungs and breathing issues. That's a good question, I would be interested to know the answer."

Some participants assumed that because it is harmful to health, it should be harmful to bones as well. Olivia had this to say,

"There are so many chemicals in cigarettes that, I would be surprised if it didn't, but that's not the first thing I think of when I think of negative health consequences of smoking." 
Some participants considered that poor bone health may be related to smoking but as an indirect consequence. Like Rose mentioned,

"Maybe indirectly, like if you are causing yourself lung damage then maybe you are going to be doing less exercise and that has an effect on your bones. I don't know if there is a direct link."

To conclude this theme, almost every participant in the focus groups was aware about the harmful effects of smoking on health, but there was general lack in knowledge regarding the ill effects of smoking on bone health.

\subsubsection{Lack of Knowledge to sequelae of poor bone health}

Another point that emerged while discussing the factors affecting peak bone mass and bone health was knowledge of the condition osteoporosis. Osteoporotic fracture is often a sequel to low bone density in adults, especially older women. This theme had two extremes, with one end of participants knowing a considerable amount about the disease and the ways to reduce the risk of osteoporotic fracture later in life, while the other group of participants had not heard anything about it except that it was something related to bones in adults. On asking participants, "what do you know about osteoporosis"?, a lot of participants came with a response that it's a disease of older people, or more so women. As Andrew replied,

"Not much really. I understand it's a bone disease that occurs mostly to women? I am not sure, I guess it also happens to men, I am not sure. Umm and it relates to, you are when you are over 40 or 50 and your bones start to get weak."

However, while most of them had heard about it or just knew that it occurred in older people, participants lacked knowledge on it as to how they could prevent this or if they should be concerned about it when they grow older. 
As Olivia from another focus group said,

"I know that's it's a thing but basically my extensive knowledge of it is that it is more common in older people and it's to do you know with bone strength and that you should drink milk. Yeah I don't have a very good understanding of like causes or preventive measures or anything like that."

A few participants had an understanding about the relationship between osteoporosis and calcium, stating that lower calcium intake in adolescence is linked to increased risk of osteoporotic fractures later in life. As Cathy said,

"I heard that maybe if you don't have enough calcium when you are younger, it can contribute to you having Osteoporosis when you are older. Because bones develop when you are younger."

While the above-mentioned views of participants represented the majority of students' opinions, there was a section of students, most of whom were from a science background, who knew more than the just basics. Like Chloe who commented about the factors affecting osteoporosis,

"Umm, so the things that I am aware of so, obviously high calcium foods, like dairy products particularly, Ummm, weight bearing exercise, that's good for bone health. There's strong genetic component to osteoporosis, and also being female, and age, various hormonal things, and menopause. And then, vitamin $D$, so in the winter, everyone is vitamin $D$ deficient, so you could take vitamin $D$ supplements."

On further exploring the knowledge behind why many of them weren't aware of osteoporosis, we had interesting answers on this. For most of the participants, it was lack of visible symptoms now, which lead to very little attention towards bone health. Participants acknowledged that because osteoporosis did not manifest in young age, they were not concerned about it in their adolescence. This led to a neglect in looking after one's own bone health in their youth. 
Like Lisa said,

"I guess because often it's something that you know it does start to get you when you get older like osteoporosis where you know just joints starting to hurt. It's usually not something we experience not necessarily in our 20's or 30's I would like to think. So we don't actively think about it, because it's not something that we're constantly engaging with.........!"

Although a lot of participants were involved in one or other form of physical activity, not many were aware that doing weight bearing exercise could have a positive effect on their peak bone mass. There were two big knowledge gaps here; firstly, many were not aware of the notion of peak bone mass and secondly several thought that there was very little one could do to increase their peak bone mass. As Zuha commented her views on peak bone mass,

"I would say may be not increase it, perhaps there's a way to keeping at same level so it doesn't decrease."

Chloe on the other hand held good information about how peak bone mass can relate to risk of osteoporotic fracture later in life and demonstrated knowledge about weight bearing exercises having a positive effect on peak bone mass,

".......My understanding was the important thing for kind of osteoporosis and bone health, not just the exercise you did, but the fact that it has to be weight bearing so for example swimming, it's good for bone health, jogging."

There were also a few participants in the focus groups who had a medical illness which increased their risk of osteoporosis. Such participants were found to be conscious about their risk and were well aware about bone health and the risk factors associated with it. Their illness lead them to more knowledge about bone health compared to their peers. Such participants were interested in learning more about the risks of osteoporosis and the lifestyle factors that could decrease the risk of osteoporotic fracture. 
As Rose said,

"I grew up eating entirely different diet from now, like lots of milk, nutella like. I was just a terrible eater; my parents were like whatever makes you full. So, like I have changed a lot and the reason why I was interested in this study at the time, in a way I have been thinking a little bit about osteoporosis. Because I have just found out that I have PCOS which in like lowest regards in terms of what is happening, one of the risks for that is high risk of osteoporosis.......!"

They were few handfuls of participants, who were maintaining a healthy diet and exercising routinely. They deciphered that they were increasing their peak bone mass, but not necessarily keeping bone health in mind. Such participants also felt the need to increase awareness and educate people about osteoporosis as this could benefit them in long run, especially regarding their bone health. As Lisa said,

"That's actually pretty interesting because it sounds like we are active, we are trying to eat healthy, I'd like to think I do spend good amount, you know, in the sun, just because I am enjoying it. And looks like it, I am doing all these things not necessarily for the purpose of bone health. So, it would be kind of really good, you know, that it would be promoted because you know it's really good to know I am actually doing something for my bones even though I wasn't as aware of it. I think it's good."

Participants acknowledged that they would benefit from more education on bone health, be it by their GP or as curriculum in University education. As Olivia said,

"I mean, I guess if I was told, like by my GP or something that I am on track to develop osteoporosis or I am not doing I should be. Then I would be happy to like add something extra to my diet to help or maybe if there is like a particular type of exercise that would be better maybe incorporate that into my routine." 
On closure for this theme, there was scant knowledge about bone health and the consequences that follows it. There was a huge knowledge gap in most of the participants had little awareness about the risks of developing osteoporotic fractures. However, a lot of participants expressed their interest in learning about it and incorporating healthy lifestyle so as to decrease their risk of fractures later in life.

\subsubsection{Menopause}

The next theme that was discussed very little in regard to knowledge about what factors affect peak bone mass, was menopause. Only two people of the total 28 participants held the knowledge about the increase in risk of osteoporotic fractures in postmenopausal women. Not many participants were aware of the protective effects of estrogen on bone in women and how the decline in estrogen levels could significantly increase the risk of osteoporotic fractures later in life. As Lea commented,

"I think I would recognize, like I would remember it if you say that because like obviously I would like had to look into it once in my life but also on top of my head there's very little that I remember about it. I definitely know it's post-menopausal though, like higher impact."

This knowledge was seen more in participants from a science background and we did not see this emerging so strongly as a theme among other participants. On further exploring her knowledge in regard to this, Lea added,

"At menopause, your hormones like drop right off. And so, I think like estrogen has really big impact on the health of the cells that lay down bone mass and I think they go down and the ones that get rid of it, stays normal. Can't remember, which cells do what, but yeah. I don't know if men are susceptible to osteoporosis, but 
testosterone probably has some impact although like they don't have menopause so it just kind of wanes over their life. So, yeah that's basically all I can think of."

This theme was not discussed in other focus groups, however participants felt that having information about an increase in risk of osteoporosis is crucial knowledge for all the women in their 20-30s, but many participants agreed that this was not discussed by their doctors till they reach menopause. It wasn't something that participants were educated about on their routine GP visits now, which further lead to a deficiency in knowledge. Hence participants agreed they knew little about this and was doing very little in their day to day life to attain a better peak bone mass.

\subsubsection{Aging \& Medical Illness}

The other minor themes that emerged to be responsible as a factor for bone health was age and associated medical illness. Quite a few participants agreed that increasing age was risk factor for deteriorating peak bone mass and osteoporotic fracture. As Zuha said,

"......... I guess in terms of bone health, ummm at high school we learned a little bit about it. And how it's important for your, yeah, I guess in the long run for your weight and your health. Especially as you grow older, your bones get more fragile and it's important to keep them healthy now."

Sharon had a medical illness and growing old was a concern to her in regard for bone health as she was aware about aftereffects of overexerting the bones and cartilages, which was more harmful then beneficial,

"Sometimes I feel like maybe I am like yeah overexerting myself because I have had obviously bone deformations and I've got some of the cartilage issues. So, I do kind of think I am going to have issues when I am old. See, I definitely think about it." 
One participant talked about poor bone health in Pacific populations and the effect it had on their health. Moana said,

" In my family we have very poor history of like health issues. I think it has a lot to do with my culture like the way my parents were brought up in the islands and the food......... A lot of my family members has passed due to health issues in the islands. And so, it wasn't just my parents, my aunties and uncles, they decided collectively...... they are going to change lifestyles. And so, we grew up pretty much well, like really well, always active and so my parents ate like that as well. And so, and specially my mum, she had diabetes, when I was quite young, when I was about 10. And now she doesn't anymore so it enabled her to be more active with like me and my dad, we would go up for a walk, and so for my mum its really special to her because she couldn't do that in the past and so she is so encouraging to keep it up in the future. You know so when I have my own family, I would be able to have to with my own husband, my children, even with my nephews and nieces just so that, having a healthy lifestyle growing up and they want to decrease my chances of getting health issues genetically but more inclined to get whatever my family had been through. So they want to decrease those chances for me and they want me to decrease those chances for next generation in my family."

Moana also added,

"I know in New Zealand the stats aren't good for Pacific Islanders and Māori so I have more motivation to help them, you know to help the wider population here and in my family and friends back home in the Islands. So yeah, it just doesn't stay within my family as well, so I want to help and be an example to everyone else because you want to be around longer, yeah."

To conclude, very few thought about the risk of fractures later in life due to osteoporosis and weren't actively thinking about improving bone health as they aged. 


\subsection{A2 Research Question II}

The following eight themes were generated pertaining to research question 2. On being asked about the factors that were barriers in day to day life, we had mixed responses to this. This part of analysis focused on the barriers faced by participants in day to day life. Participants discussed what they felt was a hindrance to them in attaining better bone health. Themes that emerged ranged from interesting factors like time management to something as elementary as weather conditions. The following table summarizes the themes and sub-themes (Table A2).

\section{Table A2: Barriers}

\section{Theme}

1. Time

2. Lack of Educational Resources

3. Student Life

4. Cost

5. Lack of Motivation

6. Cultural Factors

7. Weather

8. Alcohol \& Smoking
Sub-Theme

Transport

Work/life Balance

Lacks Knowledge

Science Background

Stress

Sedentary Lifestyle

Expensive

Guilt

Inconsistent

Social Obligation

Less Consistency

Peer Pressure 


\section{Summary}

A lot of participants stressed "time" as being a factor. For some, time management was an issue to balance work/study lifestyle, which made it difficult to find some time for physical activity while for others managing their time more judiciously not only gave them time for physical activity, but overall rendered them to be more productive in day to day life. Another impediment was transport for a few, which led to very less time left to do any kind of physical activity. The next factor that emerged was student life. University stress left little options to adopt healthy lifestyles with many wanting to stay fit but lacked the motivation to do so. Another factor that mattered was the early life environment of participants, which lead to different lifestyle habits accumulated over years and so fitting into a different environment came with its own set of obstacles. Other factors that included were being vegan by choice due to environmental reasons.

The factors that encouraged negative behaviors in day to day life were cultural factors, with smoking cigarettes and drinking alcohol being part of cultural gatherings and where peer pressure played a significant role in indulging into such activities. Cost burden also seemed to affect bone health negatively. Season affected everybody's life in some or other way with, acting either as a barrier or enabler. Lastly, lack of educational resources was a major factor for quite a few with many admitting that they wished to stay healthy but lacked knowledge and information for attaining better bone health.

These themes are discussed below in detail:

\subsubsection{Time}

In today's fast paced life, every minute on the clock counts. And this emerged as one of the major lifestyle barriers. Lots of participants expressed their desire to stay healthy and fit but felt that their busy lifestyle was a hindrance to attain not just bone health but 
overall health. Many felt that university stress, studies and work left them with very little time to eat healthily or do some sort of physical activity. As Andrew said,

"Like the busyness of the lifestyle sometimes, you just don't have time to exercise or you settle for takeaway when you could have cooked something healthier or so yeah time. But I guess it's time and the routines of work and life balance and those things."

There were also young parents in the focus groups, who had an added responsibility of looking after a child, which left them with very little time for themselves. Participants said maintaining a healthy lifestyle required dedication towards fitness by incorporating exercise in their daily lives and also cooking healthy food too required time and effort. Such participants did care about their health, but due to added responsibilities of family, their health took a back seat. As Lea said,

"...... And then with exercise, it's like having to be at Uni all the time, and then also having to have look after a child all the time, there's not heaps of time in between to think about my health right now. So, it's very much like pushed to the side, it's something I care about but like......"

Also, for a few participants, a major portion of the day was spent travelling. For somebody who lived far away from University, it took them lot of time to reach University and get back. These participants spent a lot of time on the road which left them with very little time for exercise like going to the gym. Such participants felt that distance along with time acted as a barrier. As Trish pointed out,

"I got the membership for a year but the reason I probably don't go as much as I should is I live in [location removed for confidentiality] so like I got this gym membership at Uni because it was cheaper but I have to travel in, if I am not really at Uni or if I am I have to carry all my stuff. So last year when I was in the hall, I was at the Uni gym and I went a lot more frequently. Just because it was right there, and I was in the hall so it was literally 
on campus pretty much. Yes, I don't think, I think for me the distance, the cost of it isn't, the deterrent is the distance."

Thus, most of the participants perceived time as a negative barrier. It was a hindrance to attaining better bone health in one or the other way. However, there were a few participants for whom time acted as an enabler to attain a healthy lifestyle. For such participants, their attitude towards trying to be healthy by working out rendered them more organized and enabled them to manage their time efficiently. They felt if one has a strong will to do it, one eventually finds a way to do it. Besides, exercising frequently was associated with many health benefits that not doing left them with a feeling of losing these benefits. As Olivia said,

"It's probably slightly different for me because I am in post grad, so I like don't have you know like 4 or 5 classes a day. But its lot easy for me to set up my own structure. But I used to be like I am too busy or too tired so I will do it later but I sort of recently realized that you are not actually ever that busy that you can't find an hour 3 or 4 times a week to do this. And it just improves like everything like my mood, my ability to think and all that kind of stuff. Also, if I am tired it actually you know helps. So, I find that very easy to motivate myself to go."

To sum it up, time emerged as a barrier for some to attain better bone health and healthy lifestyle. With work and studies overhead, participants felt that they were left with very little time to engage in lifestyle factors that had positive impact on bone health such as working out in gym, jogging or cycling and cooking a healthy meal. Travelling time added to this factor, with many concurring difficulties in maintaining a work/life balance. However, for a small section of participants, time acted as an enabler, where it left participants more productive by learning to manage their time more efficiently. 


\subsubsection{Lack of Educational Resources}

Another theme that emerged as a barrier in attaining better bone health, which was a very important theme and echoed in all focus groups, was the lack of available educational resources about bone health. Almost every participant agreed that they lacked knowledge about the factors that had a positive and negative impact on bone health. Calcium rich foods were hugely commented on, as many believed there should be more to bone health than just dairy. As Lisa said,

"....... When I was a kid, I know of so many advertisements and I was like, have this milk, this milk looks really good because this milk has calcium in it, it's gonna make your bones strong. But I guess you know, like everything in science, there must be more to bone health then just calcium. But I wouldn't really be aware of."

Participants reported that they did not know of many calcium rich foods except dairy. It was probably the only source of information many had and admitted there was a significant knowledge gap about other calcium rich foods. As Zuha commented,

"Yeah, I would say so. Apart from calcium, and yeah you can buy specially design milk that have added levels of calcium and Vitamin D and things. There's isn't that much information that I know personally, yeah that would impact bone health."

Calcium intake was a major problem faced by many students, especially vegans, who had cut dairy out of their diet. One of the major barriers these participants faced was that they lacked information regarding which food products were rich in calcium apart from dairy. 
As quoted by Kate,

"Dietary restrictions I think, in terms of calcium like, that's big. Like, I don't know really know that much about what to eat other than diary to get a lot of calcium. I think there has been so much in media about diary like yeah kinda all that yoghurt or cheese or whatever those commercial, they are like, you gotta get your diary, you gotta get your calcium but, yeah."

Many acknowledged that there were not many educational resources around for healthy food options and this seemed a barrier for many. As Lea said,

"Yeah time and like, I don't know like educational resources cause, you know, like you said, everything's like cheese and yoghurt, but for people who want to move towards more vegan type diet is hard to know. Like, I have no idea if there is a nutrient in tofu that's really good for it, but that would be good to know and things like that."

A few participants who had made a conscious decision to be a vegan, supplement their diet with calcium supplements to keep their calcium levels optimum and bones healthy. As Kate mentioned,

"I don't really drink a lot of milk or dairy. So, I have in the past, had some of those, the calcium chews that are like really yummy but yeah, I am also just trying to eat lot of broccoli, apparently that's good for calcium."

On exploring further, if the participants had learned about bone health in high school, college or University, they had had a brief overview that superficially touched upon bone health. For the students who did have knowledge, it was due to their personal interest in the subject or if they had a science background. 
As Lea voiced her opinion,

".... I did biomed for my undergrad, and so that is like entirely focused on human health. And I had one lecture in my entire three years on bone health, and that was the only time I heard about it as well."

For many it was a lack of knowledge about bone health that acted as a barrier in attaining better peak bone mass. Participants did not specifically know where to look for information about bone health as it wasn't easily assessable. As Lisa commented,

"I guess at the moment it would be again it's the lack of knowledge that I am not entirely sure how I could improve it. But also, for example, I could go to the gym and get you know a body scan, that tells me exactly how much muscle mass I have and how much fat mass I have. And it's really easy for me to access. At this stretch, I wouldn't really know where to go to access information about my bone health. So, I wouldn't know in what state my bones are actually in. Maybe because I feel good, I think they are good. But I actually don't know."

Also, social media popped up in discussion, with many feeling there was biased information there and it did not help in any way about educating about osteoporosis and bone health per se. As Sharon commented,

"I feel like that's the only source where you are kind of getting told that you can only get calcium from milk products. You know, it's in your face, Fonterra group is putting it out there. Um yeah, that's almost like the only information source out there that's on the TV, on the billboards, it's always for dairy. I can stop that."

Andrew too agreed about social media being biased or showing partial information,

"The problem with social media is if you are not interested in a subject, you are not going to get any information regarding it. It will only feed you, what you are searching, or you know, you get feedback on the stuff you are engaging. But I don't get any on 
osteoporosis, but I guess that's another problem with social media. Umm, and I think I am not consciously looking for information about that, so I am sure there is, but like nationwide campaign, you know like making people aware, I haven't seen that I guess."

Some participants acknowledged that, outside of social media, they hadn't seen a public campaign around this topic, or any awareness raised, specifically in New Zealand, which could educate a lot of people about bone health. As Chloe said,

"Personally, I've taken a decision to disengage with social media because I don't think it's very good. I think it's quite negative influence and so, I don't know, I can't comment on social media. I've got a skewed view as to what information there is about regarding osteoporosis, but I can't think of lots of incidence, where I have particularly seen any kind of public health campaign around it."

In summary, most of the participants indicated that they lacked knowledge about bone health and moreover did not know where to look for educational resources about bone health. The media in general was not particularly informative regarding bone health.

\subsubsection{Student life}

The next barrier that emerged as a hindrance in attaining better peak bone mass was "student Life". A lot of participants admitted that being in University was stressful for them with studies and work pressure. The mean age of participants, who were enrolled in the university, was 28 years. A lot of participants were also working alongside their studies, which added to their stress levels. This led to less focus on one's own health. 
As Jake said,

"When things are quiet, I like to do some sort of activity 3 to 4 times a week. I Play football every Monday and yeah I like to do that If things get busy that would drop down to football on Monday and everything else has to get into whatever is stressing me out."

Some participants particularly enrolled in vigorous kind of sports to de-stress from University life and acknowledged that indulging in such activities helped in dealing with stress as well as made them healthy. As Olivia commented,

"I used to deal with stress, so last year I started taking boxing classes, it was fantastic, just to sort of being better at frustration but also I get all those good you know chemicals going when you exercise."

The next thing that seemed prevalent among University students was a sedentary lifestyle. This was especially seen in students who were from fields that required long learning hours sitting in front of the desk, rendering them stationary for long hours. As Collin said,

".........What is bad for my bone health is because I am a computer science student so, I spend a lot of time just sitting in front of my PC. I know that bad when you took a long time, just stay in a fixed position, because your bone never moves."

Many agreed that being stagnant for long hours wasn't really good for bones and had a negative impact on bone health. Andrew too had to comment about bad posture,

"Bad posture, I mean spending lot of time sitting in front of desk, that type of thing or you know like a sedentary setting. You know, like using a car to go everywhere, then you just sit in front of computer and then you don't eat the nutrients you need and rely on coffee or I don't know, energy drinks." 
For some students, living in the city came with its own set of barriers. Participants found it was more difficult to engage in different types of sports in city and that living away from city gave them more flexibility. As Cathy said,

"Yeah, I believe I stopped doing team sports when I was in high school, but I did lot of rope hopping like I kind of used that, I hop around still in my backyard. I play a bit of tennis too but since I moved to the city actually, the, I am kind of hard to transition from rural activities to doing the same thing in the city."

Living in the suburbs encouraged healthy behaviors for bone health that was sometimes difficult to attain in the city. As Anushka commented,

"....... and I lived in this like, sort of big suburb like it was kind of self contained like even hospitals, universities, you had schools, you had everything. I think there was even an airport like pretty close by but like a, but like a it was like a local airport. Yeah. So, like I think our like socializing took place during like the evening. Like you cycle together you know, like go up the hills and like just try to get away from the city sort of life. So yeah."

To sum up, being a University student and balancing study/work life was quite stressful for many participants. This had a negative impact on participants' bone health, which was further exacerbated for people who had desk jobs or study routines involving long sitting hours. Also living in city came with its own set of barriers for few students who found it hard to adapt health attitudes and behaviors for better peak bone mass.

\subsubsection{Cost}

Another theme that emerged as a barrier in attaining better bone health was cost. For a few, they found enrolling in a gym to be expensive, while for other participants that was 
just an excuse. Moreover, many wanted to enroll in different forms of physical activity, but cost was a deterrent factor. Like Jake said,

"I dropped Kung Fu in the end for costs. I was doing some Yoga for a year, which I dropped because of cost. I think I got a scholarship, at the time, so I used part of that money to sign up and then the scholarship ended, and I pulled out. So yeah, the gym, I go to the gym which is pretty cheap. Certainly, a factor I think in terms of, I think if money was endless it would be easier to sign up to a variety of different things, try new things all the time and really load up your week."

Colin, who was a student, felt that cost was a barrier to join the gym to be exercising regularly. He commented,

"......Another main reason is most of the gym or places where you need to pay the money to, yeah cost is the reason."

However, for others, they felt this is just an excuse and it was more about lifestyle choices. Some found their gym subscription to be very affordable and that wasn't a barrier. In fact, they attributed this to personal motivation and felt that one does not need money to be healthy, as they can go for a jog outdoors. Like Zuha said,

"I personally don't think going to gym is that expensive. Like you can join City Fitness, for $7 \$$ a week, and that's $1 \$$ a day. So, I mean it's not something that expensive. And then specially living in Wellington we're quite lucky, there are lots of places to go for walks and runs and lots of things. And lots of hills to climb, is a natural feather."

Another way in which cost acted as a barrier was in affording high quality food. A few participants felt that eating more healthy food costs more than the regular mainstream food found in supermarkets which is cheaper and easily available, and they attributed this expense as a barrier in attaining better bone health. 
Like Andrew said,

"Maybe another barrier would be, even if you are, if you try to get, I mean, best food products is expensive............. Cost does matter. I mean if just to eat, as healthy as you can, you know, the highest level of healthy food. It's becoming more expensive, there is a gap, the kind of food you get in mainstream food that you get in supermarkets, if you are aware of it exactly, you know the labeling, most of it is like, and you know this is not really ideal."

While other participants found that being vegan by choice was definitely more expensive, as they had moved towards more home prepared food. Home cooked food required preparing meals from the beginning. This required more ingredients and costed more comparatively. As Rose said,

"For me anyway it was stepping a lot towards more home foods. It is a bit more expensive to do."

And the last aspect of cost related to smoking or drinking alcohol. However, for smoking and alcohol, being expensive actually acted as a positive reinforcement with many participants feeling cost was a major deterrent to be able to indulge in them regularly. As Kate commented about smoking,

"Yeah the expense of it probably really big deterrent into the side being addicted to something, like it's not ideal."

Cathy had similar opinion regarding alcohol intake,

"For me, that's actually a major turn off for alcohol, is that it costs a lot."

Summing up, cost was major deterrent for attaining a healthy lifestyle. And that had negative influence on peak bone mass, because students thought twice before investing money in attending a gym or any sports club. Participants perceived it could be 
expensive to buy healthy food and so the cost burden emerged as a barrier. Also, there was a subset of highly enthusiastic participants who did not find this to be a barrier and rather an excuse, but almost every participant did acknowledge the unhealthy habits like smoking \& alcohol are less prevalent in New Zealand due to its high pricing.

\subsubsection{Lack of Motivation}

The next barrier that emerged in attaining better bone health was "lack of motivation". A lot of participants attributed this lack of motivation to get around doing exercise or any forms of physical activity that was proven to be beneficial in increasing peak bone mass. Many agreed that despite having good facilities nearby, they just lacked energy or motivation to do exercise after work hours. As Aiden said,

"I am absolutely hopeless when it comes to self-motivation. I keep finding excuses, like you know, I've done quite a bit at Uni today, and I've done quite a bit of few works and I would just like to go home and just sit there and do nothing. And I guess that's the kind of lifestyle I had for a year now and when it's time to exercise, so it's tomorrow."

Interestingly, we also had some participants who were from a medical background and were very well informed about osteoporosis and its deleterious effects on health, but still lacked the will and motivation to incorporate healthy weight bearing exercises in their day to day life. As Chloe said,

"I think largely because of laziness. I equate it to dairy anyway because I am vegetarian, so it's kind of easy and I guess again almost lazy alternative. I walk to work, because I live close to work. I don't do that much weight bearing exercise, I might prefer things like swimming, partly because, I've also got hyper mobile ligaments, so I've got very unstable ankle, so things like jogging and things, I am quite liable to sprain my ankle so I don't do things like that. Even though I know, actually I probably 
should, and my ankles are probably a bit better if I did do more that kind of stuff."

Chloe noticed there was a conflict between what one's intentions are and what one's actions are. It's not necessary that what one thinks of doing, ends up doing it and she thought this to be a barrier for her. As she commented,

"I think there's I guess always a conflict between what your intentions are, what your actions are. So, kind of your own sort of, oh I know, this is what I should be doing, but actually, I am not really bother to do it or, you know, I don't really want to take vitamin $D$ supplements every day, it's like you are indifferent to it, and I won't probably won't bother"

One participant, Colin, mentioned that he lacked the motivation to find about how to improve his bone health,

"I mean if you are not like study on science or nutrition course you wouldn't know much about it and also for our self, we don't have that motivation to search online and find about how that relation like for the for your good bone health. Yeah, so yeah."

To sum it up, this was a very interesting barrier which showed us two sides of people. On one hand, we had participants from a science background who had good knowledge about osteoporosis and bone health but despite bearing knowledge they lacked the motivation and will to incorporate those good practices in their day to day life. While on the other hand, we had participants, who lacked knowledge about bone health and at the same time admitted in having less motivation to find and read about bone health. A few participants had the intention to stay healthy but lacked the actions that lead towards attitudes and behavior encompassing better bone health, while some lacked the intention and motivation to keep their bones healthy. This shows that there is lack of general awareness about bone health which affected participant's motivation to keep 
their bones healthy and this is something that is not taken into serious consideration when they are thinking about their health.

\subsubsection{Cultural Factors}

Cultural factors emerged as a barrier impacting bone health in more negative way than positive with unhealthy habits like smoking and alcohol being more prevalent in some cultures than others. Participants reflected that although they were mindful about how unhealthy these habits are for health, they still indulged in it due to cultural or social obligations. As Jake commented,

"It's certainly growing up in UK there is a sort of a culture and a lot of my friends in high school smoked and then you have got a big sort of pub culture where you and with that comes you know this sort of comes hand in hand, having a beer inside a pub and everyone goes out and has cigarettes outside the pub and chats..."

A lot of participants had come to New Zealand from different countries and experienced the culture in New Zealand is healthier with less people opting to smoke or drink alcohol, the reason partly being cost as well. However, they acknowledged that when there is gathering of families, these habits creep in, not necessarily to enjoy the intoxicating effects but rather more as a cultural thing. As Andrew said,

"Yes, we have friends in the, like every now and then, um when we have like a big barbeque and children are playing and it's kind of long thing. Umm, sometimes there will be someone who's visiting, like a mom or dad that is staying with the family and they would have smokes, and we're like, ok can I have bit of that? But it's kind of, when you are having drinks, it's kind of one of a thing. But yeah, you are right; it's kind of, very cultural." 
And the next way in which culture influenced health was food. Different cultures have different food cuisines with less or more intake of dairy, green leafy vegetables etc. Some participants found this link interesting as they felt back home, people from their culture had different exercise and food routines, which seem to have an impact on their bone health. Colin, who was originally from China commented,

" Oh yeah for the food there is one thing I forgot to say that maybe because we eat, Chinese people eat a lot green stuff, the vegetable, we eat a lot like compared to foreigners or Kiwi people. Yeah because they eat, they eat red meat a lot, yeah but we eat less compared to them."

Colin further added that the lifestyles of Chinese people were quite different from Kiwi people, as he said,

".........The main difference is yeah the lifestyle, the Kiwi people living and the Chinese people, they are different. Because in China most the elder people they doing some exercise like in open square, you know all the elder people they practicing Tai Chi or some stuff like to help their bone health, so yeah. For Kiwi people they doing more outdoor activities like cycling or climbing mountains or swimming stuff. But we don't do much about this."

To summarize, interestingly culture had a huge role to play in bone health. Be it from unhealthy habits like drinking alcohol and smoking cigarettes to eating unhealthy food or food deficient in adequate amounts of calcium. This seemed to have more negative effect than positive on peak bone mass from socio-cultural perspective. Socio-cultural obligations did lead to increase in behaviors such as smoking cigarettes or drinking alcohol which was detrimental to bone health. 


\subsubsection{Weather}

The next theme that was discussed as a barrier to attaining better bone health in terms of doing any kind of physical sports was weather. A lot of participants found that working out in the summer was easier compared to a cold winter in Wellington. For participants, who did not go to the gym, swimming and cycling was a great way to engage in physical activity and this was more regular in summer. With winter, many reported a decreased will to go out and be able to do anything. This was definitely a hindrance to many. As Aiden said,

".... And, I come from Singapore, we are from tropics, we have like 35 degrees heat and 95\% humidity, so like, we say like, oh here comes spring. Ahh spring is nice, oh, it's too cold, l'll just stay at home. We don't exercise when winter is too cold. So, I have made lot of excuses, yeah. But I guess we do make up for it with walks. We enjoy walking and we do walks during weekends. I won't say its exercise, but I think the heart rate activates it, something like that. But yeah we do try to put in little bit of effort."

However, many participants did engage in walking everyday as commented by Aiden above but did not really count that to be an exercise routine. A lot of participants felt it was easier to be consistent with exercise in summer rather than winter. Besides, summer also opened doors to many avenues of physical sports like swimming on beautiful beaches in New Zealand, mountain cycling, jogging etc. They found that it was more difficult to be consistent with exercise in winter and had less options for different kinds of physical activity. As Andrew said,

"It's just because in summer I do swim a lot. Because I particularly like swimming and when the weather gets good, I get into the habit of going to the beach after work. You know have those really hot days, one after another." 
Thus, although be it a little, seasonal pattern did seem to have an impact on participants, with them having second thoughts of going to do exercise in bad weather. Whereas summer was more convenient for many participants and gave them plenty of options in terms of physical activity. A lot of participants engaged in some form of physical sports more in summer compared to winter in New Zealand, and this season lasts a couple of months enabling more exercise during this season. This does seem to have an impact on bone health. Thus, we could say, weather was a small but important barrier in the attainment of better bone health.

\subsubsection{Alcohol and Smoking}

These factors emerged in the knowledge section too, but more than just knowledge we saw behaviors associated with drinking alcohol and smoking cigarettes that acted as a barrier in attaining peak mass. Most of the participants related this behavior due to social reasons, with it happening mostly during social gatherings. Although participants acknowledged the effects of drinking alcohol on their health, they regarded this behavior to be temporary. As Jake had a similar opinion,

"I think you just sort of, I know, it's just, such a strong social norm I guess that you just sort of understand that as long as it's not a regular thing and you know other areas of your life, too bad then, I guess you kind of think of as a temporary effect where you know you are thinking, in the long term it is not going to damage too much."

Drinking alcohol had two aspects to it. One being that alcohol drinking is part of social culture in New Zealand and most people do not think about the ill effects of alcohol on bone mineral density or on general health. As said by Moana,

"I think in New Zealand we don't, we know about the effects of alcohol but because it's a socio, like Rugby, specially just like the 
social culture in New Zealand, we don't, it's the first thing we don't think about. Like if you think of alcohol, you think of partying, friends and celebrating, dancing. You don't think about the effects until the next morning or whatever."

The other aspect of alcohol drinking in social gatherings was social obligation or peer pressure that many felt. Participants on the younger age side agreed to this as a problem they face in social gatherings, which leads to increased consumption or they end up totally avoiding such situations to escape from ill effects of alcohol. As said by Anna,

'I would say the same, like there definitely does seem to be more pressure like if you are not drinking and they are like 'Ahh, why aren't you drinking?' So, I will be like I don't drink. 'What's wrong with you? Have a drink now!' It's like, so I sort of found that instead of maybe changing my behavior in terms of drinking, I would just be like I am not going. I would rather just avoid having the social pressure of being told to drink when I don't want to drink."

Participants felt that social pressure to drink alcohol was difficult to handle and accounted it to be a barrier for attaining better bone health. As Stella said,

"There are lots of social pressures at times. I take something that looks like a beer bottle, so no one asks me any questions."

And for the more mature participants, this problem was something they had faced when they were younger but doesn't feel peer pressure to be a factor for alcohol consumption as they mature. Max resonated with this,

"Certainly, felt it but I started studying when I was 26. Umm I went to university where all my course mates were like somewhere between 18 and 20, where this was definitely a very prevalent thing, but I was mostly not hanging out with them, so I was like yeah whatever. But you know it's definitely a thing you 
feel, it's probably a very student thing and maybe you can confirm that you know. And it does occasionally happen, but you cannot avoid hanging out with friends and you are just going to keep drinking. Not very often. But it's weird habit, I think it starts off with social reasons and you feel like you are excluded if you can't participate and all that, but I feel like as time passes you'll notice you can be social with less than few drinks, probably more social."

Moving on to smoking, many participants were aware that smoking is harmful to lungs and have been associated with a number of cancers, but people still did it due to addiction issues. On further exploring the reason for making a decision to smoke or not, many participants who did not smoke did so for health reasons, whereas a certain set of participants attributed cost as an overhead burden for smoking. Especially in New Zealand, a single pack of cigarette cost approximately \$25. Quite a few participants commented on this. Like Max said,

"I did have these occasional smoking habits in the past, but I mean I pretty much stopped since I got here. That was A because I kind of wanted to, it's always been temporary, I might as well. And $B$ because it is more expensive here, gives additional incentive to it, didn't think of bones at all."

Kate had a similar opinion,

"Yeah, the expense of it probably really big deterrent into the side being addicted to something, like it's not ideal. So, yeah."

For a few participants, smoking was more prominent in social gatherings. They only smoked when they were out with friends and not otherwise. This was not something they would do on a day to day basis, but social interaction did increase this behavior 
among a few participants. As Andrew said,

"I mean just to keep with flow, you know the social, just to, I mean you are not thinking of I have to do this to be part of, it's more like you are talking, and laughing and all of that, you just keep, you are not that aware of those things. But you know, I am from Chile, and every few years we go back for a holiday, and whenever I go back, I smoke. I meet with old friends, and you know you go out, and it's kind of a special occasion, and I realize every time I end up smoking, and then it's like why I am doing this. You know, it's kind of the old habits."

There were a few participants who had moved to New Zealand and saw a very different smoking culture here, compared to rest of the world. Jake said,

"I think, suddenly it seems to be that this side of the world the attitudes to smoking, there's less people who smoke than the UK. Suddenly before I came this side of the world I knew a lot of friends who smoke, you know smoke in cars, in the house but yeah over here I can't remember the last time or ever when someone go outside for a cigarette and yeah in general I guess when I was younger I saw people avoiding smoking in house."

Also, interestingly many noticed that smoking trends are changing significantly in New Zealand where less and less number of people are smoking, with reasons of doing so ranging from health concern to cost of cigarettes. Chloe had something very interesting to comment in this regard,

"Yeah, I think culture is changing. Aren't they? So, I remember, when I was teenager it was still kind of considered, you know cool kids that were smoking behind the bike shed at school. And there was, you know a culture if you know, drinking as a teenager......... it just doesn't seem to be the same culture. And when you are walking, so I live near Wellington college/school, you know that boy school, and you don't see the boys walking in to school smoking like you might have seen when I was a teenager, in my school walking to school smoking..... so I think 
culturally, smoking is becoming less and less of a thing. And, I think partly that's due to price. So I think cost has been a massive driver in that because like, I don't really know, how much cigarettes cost now, but I think in the UK, its 10 pounds a packet, and here someone told me, its $\$ 25$ a packet, which is you know, a huge outlay per day."

To summarize, alcohol and smoking behaviors were associated with negative impact on peak bone mass. Participants blamed peer pressure for such behaviors despite knowing the ill effects of smoking and alcohol on health. But, perhaps more importantly participants weren't aware that indulging in such behaviors not only had negative impact on one's health but also had a detrimental effect on their bone health. 


\section{Chapter 4: Discussion}

\subsection{Introduction}

Our study focused on the knowledge among adolescents about peak bone mass and the lifestyle barriers faced by them in attaining adequate peak bone mass and decreasing their risk of fracture in later life. This was a qualitative study that recruited students and staff who attended focus groups at the University. The data collected was transcribed and analyzed using thematic analysis. The codes were grouped into themes answering our two main research questions. A discussion of the main themes that emerged is shown below.

\subsection{Key Findings}

\subsubsection{Knowledge}

Research question 1: What do adolescents and young adults understand about peak bone mass and risk of osteoporotic fracture?

This question looked into the knowledge held by the participants about peak bone mass. The following seven themes emerged.

\section{Supplements \& dairy products}

Most of the participants attributed good bone health to a high dairy intake and calcium supplements. This was what most of them grew up learning but acknowledged that they learned very little more about it in their higher education. Vegans often had little knowledge of calcium resources if they cut dairy produce out of the diet. Some discussed supplementing themselves with calcium and vitamin D supplements to keep 
their calcium levels adequate. Despite having this knowledge, many participants did not focus on their bone health and lacked the information that they could use to change their peak bone mass during adolescence.

\section{Physical Activity}

This theme was discussed in depth in all focus groups. Most of the participants incorporated some form of physical activity in their daily life with an intention to keep healthy and fit, not necessarily keeping bone health in mind. Very few participants were aware about the benefits of weight bearing exercise on bone health. The main motivator for doing any sort of physical activity was the overall health benefits of exercise affecting mood, stability, focus and energy, but very few participants commented about the benefits of exercise of bones. Some participants knew about bone health, which was often a result of sports related injury that led to acquiring this knowledge from their doctor. These injuries often lead to more health advice and education from health practitioners on bone health as well as recommendations on intake of calcium rich diet and vitamin D supplements. Despite engaging in some sort of physical activity, many participants lacked knowledge about the benefits of weight bearing exercise on peak bone mass.

\section{Alcohol}

This theme was often discussed as being negatively associated with bone health. Most of the participants displayed the knowledge that heavy alcohol intake has a deleterious effect on one's overall health. In regard to bone health, many assumed it must have harmful effect on bones like it did on one's general health. However, not many were aware as to how alcohol intake can have an effect on their peak bone mass and showed an interest to learn about this more. Several participants said they avoided alcohol due 
to its immediate side effects and for its cost.

\section{Smoking}

Smoking was another theme that was discussed as being negatively associated with bone health. Like alcohol, participants were aware that cigarette smoking was injurious to health, specifically the lungs, but they lacked knowledge about smoking having an effect on bone mineral density. Some participants related indirect effects of smoking on bone, such as decreased stamina leading to less exercise, but were unsure about the relationship between smoking and bones. This theme too demonstrated a significant knowledge gap in adolescents about the negative effects of smoking on bone health.

\section{Lack of knowledge of sequelae of poor bone health}

Another important knowledge pertaining to bone health was fragility fracture. While many had heard about osteoporosis, not many knew the consequences of poor bone health later in life were fractures. Most of them thought this to be a disease of older people, however they lacked knowledge about prevention of osteoporosis. While some participants shared the knowledge that lower calcium intake is associated with risk of osteoporotic fracture later in life, they were not aware about other factors. Surprisingly, two participants knew about $\mathrm{T}$ index scores that lead to diagnosis of osteoporosis but almost all the participants lacked knowledge about the factors that had impact on osteoporosis. Some students had taken courses at Victoria University that included some discussion of these points, and this may have contributed.

\section{Menopause}

Although this theme appeared less often compared to others, it is an important theme to discuss. While most of the participants associated osteoporosis with being a disease 
of women, not many knew why women were more prone to osteoporosis. Very few participants knew about the protective role of estrogen on bones and that with menopause, the decline in estrogen levels increases the risk of osteoporotic fractures. Participants said that this was something that is not discussed by their GP until they reach menopause, leaving young women more deficient in knowledge about attaining better peak bone mass and preventing osteoporotic fractures later in life.

\section{Aging \& medical illness}

These were the minor themes that were associated with knowledge about peak bone mass. Many participants acknowledged that bones get fragile with age and that it is important to take care of them, however not many knew they could actually increase their peak bone mass with changing their lifestyle and this was something that should be addressed in young adolescents as this could significantly decrease their risk of osteoporotic fractures. Medical related illness that exacerbates the risk of osteoporotic fracture was concern for few with medical illness. However, this knowledge was due to their medical illness and not a part of general awareness.

\subsubsection{Barriers}

Research Question 2: What are the barriers that young people experience in adopting lifestyles associated with better bone health and how could we reduce this?

This question looked into lifestyle barriers faced by adolescents in their day to day life to attain better peak bone mass. The following eight themes emerged. 


\section{Time}

Time emerged as one of the important themes, especially among young adults. With a lot of them being in University or early in their career, meeting study and work demands did lead to a decrease in the time spent doing physical sports activity, be it gym, cycling, jogging etc. While many had a strong motivation to stay fit, the time factor was a hindrance to a few, who wished to stay fit but were unable to do so. Some participants had the added responsibility of family and children, which further put restrictions on concentrating on one's own health. Travelling for work only added to this barrier with more time spent on the road. In contrast, there were also participants who were very fixed and rigid with their routine, who not only managed to meet their daily deadlines but also managed to workout, which gave them a sense of achievement.

\section{Lack of Resources}

Another important factor to discuss deals with young adults' lack of knowledge about bone health and their access to information regarding it. Bone health education was not stressed in schools and colleges leading to a huge knowledge gap in young adults. A lot of participants displayed the knowledge that a good calcium intake is required for bone health. However, this was often the only knowledge they had, giving rise to a barrier for those who avoided dairy. Many felt that apart from dairy products, there was significant lack of knowledge about other calcium-rich foods. There was a knowledge gap regarding peak bone mass and the measures that could be taken to increase one's own bone mineral density in their second to third decade of life. A knowledge gap was also observed regarding inexpensive forms of physical activities. Walking, jogging or cycling kind of exercises were not considered by participants as measures of increasing their peak bone mass reflecting the knowledge gap. Participants also acknowledged that bone health isn't part of the standard curriculum widening the knowledge gap. While many did relate to information provided during health visits to a health practitioner or 
through the media, they felt this information was just too basic and did not stress peak bone mass. As for the media, participants often felt it was more biased information than educative information. Therefore, the lack of health information resources was a major barrier in attaining a better peak bone mass and if addressed correctly, could have a positive impact on bone health of many individuals who are willing to accommodate healthy lifestyle choices in their life.

\section{Sedentary student Life}

With the mean age of participants being 28 years, most of our participants were in a graduate/post-grad program and working as well. This study/work stress took a toll on some of the participants leaving them tired and reluctant to engage in any kind of physical activity. This factor was further exacerbated by sedentary lifestyle in many students, who had a desk job or study routines with long sitting hours, which wasn't really good for bones and affected peak bone mass of these young individuals.

\section{Cost}

Cost emerged as another barrier in attaining better peak bone mass. As most of the participants were students who were supporting themselves, enrolling in a gym or any sports club was an extra financial burden which limited participants' engagement in any form of physical activity, thus acting as an obstacle in achieving a better bone health. What was also significant was the knowledge gap regarding physical activities that could be achieved without being a part of any sports club. Another way cost came across as a barrier was in affording healthy foods. Participants acknowledged buying some healthy foods was expensive compared to regular mainstream supermarket foods, which were cheap and easily available compared to healthy food. On the other hand, cost had an indirect positive effect in reducing smoking and alcohol rates in New Zealand as these are highly expensive and was a huge outlay per day for many. 


\section{Lack of Motivation}

Another theme that emerged as a barrier in attaining better peak bone mass was lack of motivation. Despite having many sports clubs and activities facilities available at University, several participants reported they lacked motivation to do anything after they finished university/work. This seemed prevalent not only in general students but also in participants from a science background who had good knowledge about osteoporosis and its deleterious effect on bone. Yet, they confessed they were just too lazy to engage in healthy habits.

\section{Ethnicity/Culture}

New Zealand is a diverse country with people from different cultures and ethnicities. Although, the general trend of food cuisine is the same across New Zealand, there is quite a mix of cultural ethnicities here. And different cultures had different food cuisines which contributed positively or negatively on bone health with respect to intake of calcium rich foods. Another impact of this multiculturalism is seen in smoking cigarettes and drinking alcohol. These are deeply imbedded in cultures across the world. This seemed to reflect here in New Zealand as well with many indulging in these behaviors because of their cultural backgrounds.

\section{Seasonal Patterns}

An additional factor that could be taken into consideration is the effect of season on physical activity. Wellington is well known for being windy during winters and spring, which significantly limits physical activities for participants and acts as barrier in attaining better peak bone mass. At the same time summer provided participants with more opportunities to engage in different kinds of physical sports and maintain that consistency of healthy behavior for better bone health. Thus, winters and springs were 
associated with decreased exposure to sunshine due to more sedentary lifestyle, and therefore (albeit for short period of time), pointed towards the fact that weather has an effect on acquisition of better peak bone mass.

\section{Alcohol and smoking}

These factors emerged in the knowledge section too, but here they were associated with behaviors that were detrimental to bone health. Peer pressure seemed to play an important role that could lead to an increase in these behaviors, where participants confessed that they did not want to indulge in these social habits but found it hard to avoid them. Participants felt that drinking alcohol was a part of New Zealand culture, and was available in every social event and people indulged in it without thinking about the side effects until the next day. The same applied to cigarette smoking, where people did it more often in social gatherings despite knowing its harmful effects on health. Although participants were aware about the harmful effects of cigarette smoking and alcohol, they did indulge in them because it was the social norm.

\subsection{Study Limitation}

There were some obvious study limitations in this type of research methodology that limit our ability to draw conclusions. Firstly, the sample is small and focused on a university population, thus not allowing us to generalize to a large population. However, small sample sizes are an inherent aspect of qualitative research, and do not limit the usefulness of results in terms of relevance to the population studied. Results may also be transferable to other populations who share similar characteristics. Secondly, during the discussions, commonly one participant may talk more than others, leaving the shy participants quiet. Or, when a small group arrives at a consensual agreement regarding something, this may leave individuals with difference of opinion voiceless. Such 
situations were often handled by the researcher by moderating the discussion by keeping it open to all to view their opinion. Lastly and most importantly, all researchers tend to hold their different perspectives or positions of the research, which might lead to the development of different, although equally valid, understandings of a particular situation under study. As Malterud said, "A researcher's background and position will affect what they choose to investigate, the angle of investigation, the methods judged most adequate for this purpose, the findings considered most appropriate, and the framing and communication of conclusions" (101).

However, this can be overcome by designing a research with multiple investigators, which can lead to dialogue, lead to the development of complementary as well as divergent understandings of a study situation and provide a context in which researchers' often hidden - beliefs, values, perspectives and assumptions can be revealed and contested. Also maintaining a reflexive journal, and making regular entries during the entire research process, helps the researcher to be grounded with the study (Lincoln, YS. \& Guba, EG. (1985), Naturalistic Inquiry).

\subsection{Adolescents and bone health: the importance of this work}

In one's life, around $46-62 \%$ of BMD is genetically determined and heritable(102) while the lifestyle factors account for the rest. The period of most rapid skeletal development occurs in childhood and adolescence which accounts for $30-40 \%$ of total bone mass increase $(103,104)$. Lifestyle choices attribute to $40 \%$ to $60 \%$ of peak bone mass of an individual $(105,106)$. Many studies have been conducted about the beneficial effects of weight bearing exercises(70) as well as intake of calcium supplements(107) having a positive impact on BMD. However, very few studies looked into other lifestyle factors affecting peak bone mass. Specifically, physical activity during the growth period is associated with higher bone mass accrual in adolescents(108) and that there is a dose response relationship between physical activity and bone mass(69). Participants in our 
focus groups were aware of the health benefits of physical activity and this emerged significantly in all focus groups. However, very few were aware about the association between weight bearing exercises and peak bone mass, leading to an intervention opportunity in this age group(70). There was a significant knowledge gap in young adults about how weight bearing exercise during pubertal age could help increase peak bone mass in adolescents $(70,109)$. Many studies have investigated this relationship in young adolescents and demonstrated that peak bone mass accrual is more strongly associated in males than females with physical activity(104) and other factors such as calcium and vitamin $D$ consumption, and this information could be conveyed to this age group to inspire healthy behaviors.

There are a few studies that have been conducted to look into perceptions towards calcium and vitamin D intake. One Canadian study found that seventy-eight per cent of young adults who consumed inadequate vitamin D perceived their intake as adequate, compared with fifty-seven per cent for calcium, when they were actually consuming inadequate amounts (3). In our study, there was definitely knowledge about the beneficial effects of calcium and vitamin $D$ on bone health, however many participants equated the intake of calcium with dairy. The consumption of only dairy products for calcium represented the knowledge among university students and they lacked the knowledge on alternative sources of calcium. Another Australian study looked into barriers and enablers into achieving adequate calcium intake in young adults. They found that young adults had limited knowledge of sources, prevention of disease and recommended amounts(2). Our findings of this study supported this as students lacked knowledge on alternative sources of calcium apart from dairy and vegans faced a huge barrier in keeping their calcium sources adequate as they lacked knowledge on alternative sources. We need more studies to look into attitudes and perception of better bone health that can help us implement healthy attitudes and behaviors among adolescents that could significantly impact the rates of osteoporotic fractures in future generations. 
Alcohol consumption has been a part of history worldwide for generations and New Zealand is not an exception. A standard drink in New Zealand contains $10 \mathrm{~g}$ of ethanol, even though there is disparity over Governmental standard drink definitions, stated by a recent study of low risk alcohol consumption in 75 countries(110). New Zealand, like many places in the world, has a culture of binge drinking, which is particularly prevalent among adolescents, who drink more often than their nonstudent peers(111). The MPLA for alcohol is 18 years and so most university students can obtain alcohol legally. Approximately $50 \%$ of boys and $40 \%$ of girls have had an alcoholic drink by age 13 . Almost all epidemiological studies of alcohol use on bone health showed that heavy alcohol drinking during adolescence and young adulthood can effect bone mineral density and increase the risk of osteoporotic fracture later in life(107). Previous studies have demonstrated the deleterious effect of alcohol on bone formation that resulted in slow bone formation, aberrant remodeling of skeletal tissue and, ultimately, osteopenia and fractures(112). But very few have looked into the attitudes and behaviors of young adults on alcohol consumption in regard to bone health. Our study revealed significant deficiency in knowledge about the effects of alcohol on bone health. While our participants were aware that drinking alcohol is associated with many adverse health effects, not many were aware about the effects of alcohol on bone health. While most of the participants assumed that it might be harmful to bones as well, none were informed about this aspect of alcohol consumption.

While many studies have been conducted among older people, very few studies have looked into the effects of alcohol consumption in young adults. A Korean study found low BMD in young adolescent girls who consumed alcohol compared to their healthy peers, thus calling for more studies like ours in future to raise awareness about the ill effects of alcohol consumption on bone health(113). A qualitative study from the Netherlands looked into attitudes towards alcohol consumption in adolescent population and found that curiosity was an important factor for starting alcohol drinking followed by peer pressure, that played an important role in drinking alcohol(114). 
Parental role was also reported to be a significant factor for limiting alcohol intake in adolescents in this study(114). This was also a finding in our study where participants reported peer pressure to be an important factor for indulging in drinking behavior. Lack of knowledge about bone health and other health conditions compounded this behavior among young adults. More education and awareness about alcohol consumption and its detrimental effects on not just bone health but overall health could help reduce risky drinking behavior among young adolescents, which could significantly lead to less bone loss during early years and better bone mass accrual.

Smoking is one of the biggest public health threats the world has ever faced. It is estimated that around 1.1 billion people smoke tobacco as per the Global Health Observatory (GHO) date released by WHO. Apart from the known harmful effects of smoking on other organs, cigarette smoking is associated with reduced bone mineral density with an increased risk of fracture later in life(115). Tobacco exerts its deleterious effect by interfering with bone metabolism and decreases bone mineral density by increasing osteoclastic resorption of bone(115). This effect observed is due to the antiestrogenic affect seen in smokers.

The Annual Data Explorer shows 2016/17 results from the continuous New Zealand Health Survey estimates 600,000 adults to be smokers (18). Smoking rates in New Zealand Aotearoa continue to reduce, with $16 \%$ of adults currently smoking (this has dropped from $25 \%$ in 1996/97).

Likewise, many clinical and observational studies have looked into the effects of smoking on bone mineral density. Importantly a meta-analysis reported that past smoking history was significantly associated with high future fracture risk(96). Early initiation of smoking in adolescents was detrimental to bone mineral density(89). Our study is first of its kind to look in lifestyle factors and the barriers associated with attaining a better peak bone mass. Smoking behavior in students was more common in social situations rather than an addictive habit. Students in our study reported that 
indulging in cigarette smoking was augmented by peer pressure despite being aware of its ill effects on health, and this behavior was more evident in social settings. However, bone health was not a consideration for many who smoked with participants stating they lacked knowledge about the effects of smoking on bone health. Cigarette cost did act as enabler for less people smoking in New Zealand, but the knowledge gap existed among adolescents. This calls for increasing awareness about the negative factors, like alcohol and smoking, that has a deleterious effect on peak bone mass. There is need to educate young adults about the lifestyle factors that have a significant impact on peak bone mass so as to encourage more healthy behaviors and attitudes among young population.

\subsection{Future Directions}

\section{Summary}

Lifestyle choices account for $20-40 \%$ of adult peak bone(30). By early in the third decade of life, most adults have attained their peak bone mass(33) that determines their risk of osteoporotic fracture. While $40-60 \%$ of the peak bone mass is inherited genetically(102), the rest depends on the individuals lifestyle factors. The National Osteoporosis foundation recommend lifestyle choices from childhood through young to late adulthood that provide evidence based beneficial effects of calcium and vitamin $D$ supplementation on bone mass accrual as well as on role of physical activity on peak bone mass in these growing years(30). However, more data are needed to look into lifestyle choices affecting bone health. Our study is the first of its kind to look into this from a qualitative point of view. We looked into the knowledge among young adults with regard to bone mass and found a significant knowledge gap. Participants were not aware about bone mass accrual in their adolescent years and the factors that had an 
impact on it. Our findings chime with a recent Australian study looked into barriers and enablers for achieving adequate calcium intake in young adults and found that young adults had limited knowledge of sources, prevention of disease and recommended amounts(2). We looked into lifestyle barriers for attaining better peak bone mass in young adults and found participants had limited knowledge about resources for exercise, calcium rich foods, alternatives to dairy and adequate vitamin $D$ being beneficial. Participants also lacked significant information on osteoporosis and the ways to prevent the disease in future. This finding also echoes with one study done to assess the knowledge about osteoporosis, where they found that there was serious deficits in knowledge among healthy and diagnosed men and women with osteoporosis, as well as among health professionals(116). This study also reported that educational interventions were accompanied by an increase in knowledge, but no change in behavior(116). We also found there was a significant knowledge gap on recommended amounts of calcium and vitamin $\mathrm{D}$ one should take to attain better peak bone mass. Barriers in day to day life ranged from stressful life leading to very less time to engage in healthy behaviors. Being vegan also sometimes acted as a barrier. The cost of gym membership and lack of motivation also added to barriers to attaining better bone health, but more so much of the behavior and attitude seen in young adults was due to lack of knowledge on bone health and its sequelae later in life.

\section{Recommendations}

Studies have shown that there is a linear relationship between health literacy and health behaviors adopted by adolescents(117). This calls for a multi sectored approach to increase literacy across schools, universities and healthcare facilities to educate the young masses about bone health by applying recommendations of dietary and physical activity guidelines that could lead adolescent to adopt healthy lifestyle behaviors. Detailed guidelines and strategies might be constructed to improve and implement standard nutrition and daily physical activity guidelines. Schools and Universities could 
actively try to implement these guidelines in their curriculum with reducing work/study stress on students by incorporating sports between classroom hours. Healthcare workers could endeavor to counsel and educate young adults about bone health, nutrition and physical activity as well as educating them about ill effects of smoking and alcohol. Government could provide tool kits and educational material with an active display around schools, universities and health clinics with consistent messaging about bone health and the factors impacting it. The growth of social media has opportunities for benefitting public health by influencing and educating users. Capacity building by educating social media users and organizational policies that support social media use may help achieve this(118).

\subsection{Conclusion}

The aim of this thesis was to look into knowledge about bone health in young adults and the barriers faced by them in attaining better peak bone mass. We found a significant knowledge gaps in these young adolescents about peak bone mass and the factors that affect it. Most of them equated adequate calcium intake with dairy intake. They lacked knowledge of alternative food sources of calcium. Adolescents identified osteoporosis to be a disease of old people and did not consider their own bone mineral density status to be a risk factor for attaining fractures. There was also a significant knowledge gap about peak bone mass and the ways to increase it during early years. Lifestyle factors acted as barriers in attaining adequate peak bone mass. These deficits of knowledge should be addressed by providing updated and reliable information through appropriate health promotion. While many studies have been undertaken on factors such as physical activity, calcium and vitamin D supplementation to dairy intake, very few studies have actually looked into lifestyle factors and the barriers faced by young adolescents in their everyday life in achieving better peak bone mass. In fact, to our knowledge, ours is the first qualitative study to look into barriers to adoption of lifestyle in attaining optimal peak bone mass. More research is needed to better understand 
lifestyle factors that are less clearly understood for building better bone health. At the moment, meeting recommended guidelines for intake of nutrients and physical activity while cautioning against harmful behaviors is a priority. Implementing the above strategies could significantly lead to lower rates of osteoporotic fractures in the coming generations, thus lowering the burden of osteoporosis.

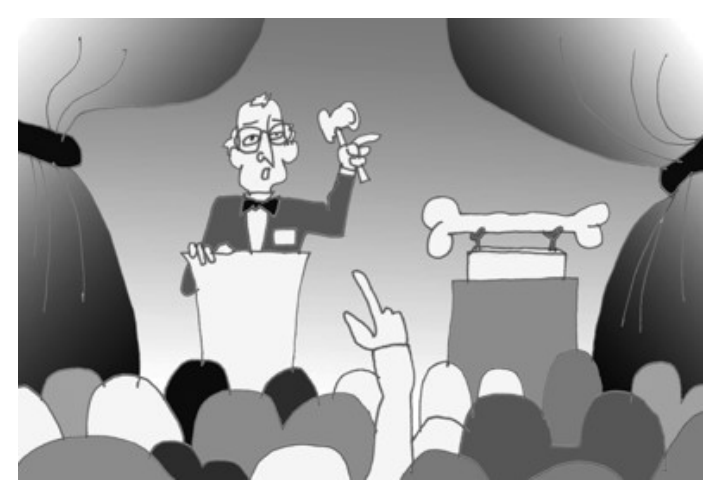




\section{References}

1. Glaser DL, Kaplan FS. Osteoporosis. Definition and clinical presentation. Spine (Phila Pa 1976). 1997;22(24 Suppl):12s-6s.

2. Rouf $A$, Clayton $S$, Allman-Farinelli $M$. The barriers and enablers to achieving adequate calcium intake in young adults: a qualitative study using focus groups. J Hum Nutr Diet. 2019.

3. Holland A, Moffat T. Comparing measured calcium and vitamin D intakes with perceptions of intake in Canadian young adults: insights for designing osteoporosis prevention education. Public Health Nutr. 2017;20(10):1760-7.

4. Organization WH. <Osteoporosis WHO.pdf>. 2004.

5. Office of the Surgeon G. Reports of the Surgeon General. Bone Health and Osteoporosis: A Report of the Surgeon General. Rockville (MD): Office of the Surgeon General (US); 2004.

6. van Staa TP, Dennison EM, Leufkens HG, Cooper C. Epidemiology of fractures in England and Wales. Bone. 2001;29(6):517-22.

7. Cooper C, Campion G, Melton LJ, 3rd. Hip fractures in the elderly: a world-wide projection. Osteoporosis international : a journal established as result of cooperation between the European Foundation for Osteoporosis and the National Osteoporosis Foundation of the USA. 1992;2(6):285-9.

8. Dr Paul Brown DRM, Eman Radwan, Jane Willingale. BONZ Report. 2007.

9. Sambrook PN, Seeman E, Phillips SR, Ebeling PR. Preventing osteoporosis: outcomes of the Australian Fracture Prevention Summit. The Medical journal of Australia. 2002;176 Suppl:S116.

10. Hannan MT, Felson DT, Dawson-Hughes B, Tucker KL, Cupples LA, Wilson PW, et al. Risk factors for longitudinal bone loss in elderly men and women: the Framingham Osteoporosis Study. Journal of bone and mineral research : the official journal of the American Society for Bone and Mineral Research. 2000;15(4):710-20.

11. O'Neill TW, Felsenberg D, Varlow J, Cooper C, Kanis JA, Silman AJ. The prevalence of vertebral deformity in european men and women: the European Vertebral Osteoporosis Study. Journal of bone and mineral research : the official journal of the American Society for Bone and Mineral Research. 1996;11(7):1010-8.

12. Curtis EM, van der Velde R, Moon RJ, van den Bergh JP, Geusens P, de Vries F, et al. Epidemiology of fractures in the United Kingdom 1988-2012: Variation with age, sex, geography, ethnicity and socioeconomic status. Bone. 2016;87:19-26.

13. Nicholas C. Harvey EMC, Elaine M. Dennison,, Cooper aC. <The epidemiology of osteoporotic fractures..pdf>. 2019.

14. Cooper C, Cole ZA, Holroyd CR, Earl SC, Harvey NC, Dennison EM, et al. Secular trends in the incidence of hip and other osteoporotic fractures. Osteoporosis international : a journal established as result of cooperation between the European Foundation for Osteoporosis and the National Osteoporosis Foundation of the USA. 2011;22(5):1277-88.

15. Lofthus CM, Osnes EK, Falch JA, Kaastad TS, Kristiansen IS, Nordsletten L, et al. Epidemiology of hip fractures in Oslo, Norway. Bone. 2001;29(5):413-8.

16. Rockwood PR, Horne JG, Cryer C. Hip fractures: a future epidemic? J Orthop Trauma. 1990;4(4):388-93.

17. Fielden J, Purdie G, Horne G, Devane P. Hip fracture incidence in New Zealand, revisited. N Z Med J. 2001;114(1129):154-6. 
18. Brown $P$, McNeill R, Leung W, Radwan E, Willingale J. Current and future economic burden of osteoporosis in New Zealand. Applied health economics and health policy.

2011;9(2):111-23.

19. Klop C, Welsing PM, Cooper C, Harvey NC, Elders PJ, Bijlsma JW, et al. Mortality in British hip fracture patients, 2000-2010: a population-based retrospective cohort study. Bone. 2014;66:171-7.

20. Bliuc D, Nguyen ND, Milch VE, Nguyen TV, Eisman JA, Center JR. Mortality risk associated with low-trauma osteoporotic fracture and subsequent fracture in men and women. Jama. 2009;301(5):513-21.

21. Katsimbri P. The biology of normal bone remodelling. Eur J Cancer Care (Engl). 2017;26(6).

22. Mercado-Pagan AE, Stahl AM, Shanjani Y, Yang Y. Vascularization in bone tissue engineering constructs. Ann Biomed Eng. 2015;43(3):718-29.

23. Kapinas K, Delany AM. MicroRNA biogenesis and regulation of bone remodeling. Arthritis Res Ther. 2011;13(3):220.

24. Riddle RC, Clemens TL. Bone Cell Bioenergetics and Skeletal Energy Homeostasis. Physiological reviews. 2017;97(2):667-98.

25. Rucci N. Molecular biology of bone remodelling. Clinical cases in mineral and bone metabolism : the official journal of the Italian Society of Osteoporosis, Mineral Metabolism, and Skeletal Diseases. 2008;5(1):49-56.

26. Almeida M, Laurent MR, Dubois V, Claessens F, O'Brien CA, Bouillon R, et al. Estrogens and Androgens in Skeletal Physiology and Pathophysiology. Physiological reviews. 2017;97(1):135-87.

27. Raggatt LJ, Partridge NC. Cellular and molecular mechanisms of bone remodeling. J Biol Chem. 2010;285(33):25103-8.

28. Matkovic V, Jelic T, Wardlaw GM, llich JZ, Goel PK, Wright JK, et al. Timing of peak bone mass in Caucasian females and its implication for the prevention of osteoporosis. Inference from a cross-sectional model. J Clin Invest. 1994;93(2):799-808.

29. Bonjour JP, Chevalley T, Ferrari S, Rizzoli R. The importance and relevance of peak bone mass in the prevalence of osteoporosis. Salud publica de Mexico. 2009;51 Suppl 1:S5-17.

30. Weaver CM, Gordon CM, Janz KF, Kalkwarf HJ, Lappe JM, Lewis R, et al. The National Osteoporosis Foundation's position statement on peak bone mass development and lifestyle factors: a systematic review and implementation recommendations. Osteoporosis international : a journal established as result of cooperation between the European Foundation for Osteoporosis and the National Osteoporosis Foundation of the USA. 2016;27(4):1281-386.

31. Davies JH, Evans BA, Gregory JW. Bone mass acquisition in healthy children. Arch Dis Child. 2005;90(4):373-8.

32. Abrams SA. Normal acquisition and loss of bone mass. Horm Res. 2003;60 Suppl 3:71-6.

33. Baxter-Jones AD, Faulkner RA, Forwood MR, Mirwald RL, Bailey DA. Bone mineral accrual from 8 to 30 years of age: an estimation of peak bone mass. Journal of bone and mineral research : the official journal of the American Society for Bone and Mineral Research. 2011;26(8):1729-39.

34. Yakar S, Isaksson O. Regulation of skeletal growth and mineral acquisition by the GH/IGF-1 axis: Lessons from mouse models. Growth Horm IGF Res. 2016;28:26-42.

35. Heaney RP, Abrams S, Dawson-Hughes B, Looker A, Marcus R, Matkovic V, et al. Peak bone mass. Osteoporosis international : a journal established as result of cooperation between the European Foundation for Osteoporosis and the National Osteoporosis Foundation of the USA. 2000;11(12):985-1009. 
36. Parfitt AM. The two faces of growth: benefits and risks to bone integrity. Osteoporosis international : a journal established as result of cooperation between the European Foundation for Osteoporosis and the National Osteoporosis Foundation of the USA. 1994;4(6):382-98.

37. Cooper C, Dennison EM, Leufkens HG, Bishop N, van Staa TP. Epidemiology of childhood fractures in Britain: a study using the general practice research database. Journal of bone and mineral research : the official journal of the American Society for Bone and Mineral Research. 2004;19(12):1976-81.

38. Ferrari SL, Chevalley T, Bonjour JP, Rizzoli R. Childhood fractures are associated with decreased bone mass gain during puberty: an early marker of persistent bone fragility? Journal of bone and mineral research : the official journal of the American Society for Bone and Mineral Research. 2006;21(4):501-7.

39. Christoffersen T, Emaus N, Dennison E, Furberg AS, Gracia-Marco L, Grimnes G, et al. The association between childhood fractures and adolescence bone outcomes: a populationbased study, the Tromso Study, Fit Futures. Osteoporosis international : a journal established as result of cooperation between the European Foundation for Osteoporosis and the National Osteoporosis Foundation of the USA. 2018;29(2):441-50.

40. Landin LA. Fracture patterns in children. Analysis of 8,682 fractures with special reference to incidence, etiology and secular changes in a Swedish urban population 1950-1979. Acta Orthop Scand Suppl. 1983;202:1-109.

41. Mayranpaa MK, Makitie O, Kallio PE. Decreasing incidence and changing pattern of childhood fractures: A population-based study. Journal of bone and mineral research : the official journal of the American Society for Bone and Mineral Research. 2010;25(12):2752-9. 42. Clark EM, Ness AR, Tobias JH. Vigorous physical activity increases fracture risk in children irrespective of bone mass: a prospective study of the independent risk factors for fractures in healthy children. Journal of bone and mineral research : the official journal of the American Society for Bone and Mineral Research. 2008;23(7):1012-22.

43. Loud KJ, Gordon CM, Micheli LJ, Field AE. Correlates of stress fractures among preadolescent and adolescent girls. Pediatrics. 2005;115(4):e399-406.

44. Hame SL, LaFemina JM, McAllister DR, Schaadt GW, Dorey FJ. Fractures in the collegiate athlete. Am J Sports Med. 2004;32(2):446-51.

45. Loud KJ, Micheli LJ, Bristol S, Austin SB, Gordon CM. Family history predicts stress fracture in active female adolescents. Pediatrics. 2007;120(2):e364-72.

46. Kelsey JL, Browner WS, Seeley DG, Nevitt MC, Cummings SR. Risk factors for fractures of the distal forearm and proximal humerus. The Study of Osteoporotic Fractures Research Group. Am J Epidemiol. 1992;135(5):477-89.

47. Nelson HD, Haney EM, Chou R, Dana T, Fu R, Bougatsos C. U.S. Preventive Services Task Force Evidence Syntheses, formerly Systematic Evidence Reviews. Screening for Osteoporosis: Systematic Review to Update the 2002 US Preventive Services Task Force Recommendation. Rockville (MD): Agency for Healthcare Research and Quality (US); 2010.

48. Slemenda CW, Christian JC, Williams CJ, Norton JA, Johnston CC, Jr. Genetic determinants of bone mass in adult women: a reevaluation of the twin model and the potential importance of gene interaction on heritability estimates. Journal of bone and mineral research : the official journal of the American Society for Bone and Mineral Research. 1991;6(6):561-7. 49. Smith DM, Nance WE, Kang KW, Christian JC, Johnston CC, Jr. Genetic factors in determining bone mass. J Clin Invest. 1973;52(11):2800-8.

50. Zhou B, Wang J, Stein EM, Zhang Z, Nishiyama KK, Zhang CA, et al. Bone density, microarchitecture and stiffness in Caucasian and Caribbean Hispanic postmenopausal American women. Bone research. 2014;2:14016. 
51. Eisman JA. Vitamin D receptor gene alleles and osteoporosis: an affirmative view. Journal of bone and mineral research : the official journal of the American Society for Bone and Mineral Research. 1995;10(9):1289-93.

52. Estrada K, Styrkarsdottir U, Evangelou E, Hsu YH, Duncan EL, Ntzani EE, et al. Genomewide meta-analysis identifies 56 bone mineral density loci and reveals 14 loci associated with risk of fracture. Nat Genet. 2012;44(5):491-501.

53. Gross C, Musiol IM, Eccleshall TR, Malloy PJ, Feldman D. Vitamin D receptor gene polymorphisms: analysis of ligand binding and hormone responsiveness in cultured skin fibroblasts. Biochemical and biophysical research communications. 1998;242(3):467-73.

54. Peacock M. Vitamin D receptor gene alleles and osteoporosis: a contrasting view. Journal of bone and mineral research : the official journal of the American Society for Bone and Mineral Research. 1995;10(9):1294-7.

55. Rubin LA, Hawker GA, Peltekova VD, Fielding LJ, Ridout R, Cole DE. Determinants of peak bone mass: clinical and genetic analyses in a young female Canadian cohort. Journal of bone and mineral research : the official journal of the American Society for Bone and Mineral Research. 1999;14(4):633-43.

56. Reifenstein EC, Jr., Albright F. The classic: the metabolic effects of steroid hormones in osteoporosis. 1946. Clinical orthopaedics and related research. 2011;469(8):2096-127.

57. Gennari L, Nuti R, Bilezikian JP. Aromatase activity and bone homeostasis in men. The Journal of clinical endocrinology and metabolism. 2004;89(12):5898-907.

58. Rizzoli R, Bonjour J-P. Hormones and bones. The Lancet. 1997;349:S20-S3.

59. Seeman E. Osteoporosis in men. Baillieres Clin Rheumatol. 1997;11(3):613-29.

60. Kameda T, Mano H, Yuasa T, Mori Y, Miyazawa K, Shiokawa M, et al. Estrogen inhibits bone resorption by directly inducing apoptosis of the bone-resorbing osteoclasts. The Journal of experimental medicine. 1997;186(4):489-95.

61. Tomkinson A, Reeve J, Shaw RW, Noble BS. The death of osteocytes via apoptosis accompanies estrogen withdrawal in human bone. The Journal of clinical endocrinology and metabolism. 1997;82(9):3128-35.

62. Manolagas SC. Birth and death of bone cells: basic regulatory mechanisms and implications for the pathogenesis and treatment of osteoporosis. Endocrine reviews. 2000;21(2):115-37.

63. DeCherney A. Bone-sparing properties of oral contraceptives. Am J Obstet Gynecol. 1996;174(1 Pt 1):15-20.

64. Allali F, El Mansouri L, Abourazzak F, Ichchou L, Khazzani H, Bennani L, et al. The effect of past use of oral contraceptive on bone mineral density, bone biochemical markers and muscle strength in healthy pre and post menopausal women. BMC Womens Health. 2009;9:31. 65. Zhang MH, Zhang W, Zhang AD, Yang Y, Gai L. Effect of depot medroxyprogesterone acetate on bone mineral density in adolescent women. Chin Med J (Engl). 2013;126(21):4043-7. 66. Vanderschueren D, Vandenput L, Boonen S, Lindberg MK, Bouillon R, Ohlsson C. Androgens and bone. Endocrine reviews. 2004;25(3):389-425.

67. Sozen T, Ozisik L, Basaran NC. An overview and management of osteoporosis. European journal of rheumatology. 2017;4(1):46-56.

68. Cosman F, de Beur SJ, LeBoff MS, Lewiecki EM, Tanner B, Randall S, et al. Clinician's Guide to Prevention and Treatment of Osteoporosis. Osteoporosis international : a journal established as result of cooperation between the European Foundation for Osteoporosis and the National Osteoporosis Foundation of the USA. 2014;25(10):2359-81. 
69. Christoffersen T, Winther A, Nilsen OA, Ahmed LA, Furberg AS, Grimnes G, et al. Does the frequency and intensity of physical activity in adolescence have an impact on bone? The Tromso Study, Fit Futures. BMC Sports Sci Med Rehabil. 2015;7:26.

70. Hind $\mathrm{K}$, Burrows $\mathrm{M}$. Weight-bearing exercise and bone mineral accrual in children and adolescents: a review of controlled trials. Bone. 2007;40(1):14-27.

71. Lanyon LE. Control of bone architecture by functional load bearing. Journal of bone and mineral research : the official journal of the American Society for Bone and Mineral Research. 1992;7 Suppl 2:S369-75.

72. Lanyon LE, Rubin CT. Static vs dynamic loads as an influence on bone remodelling. J Biomech. 1984;17(12):897-905.

73. Robling AG, Burr DB, Turner $\mathrm{CH}$. Recovery periods restore mechanosensitivity to dynamically loaded bone. J Exp Biol. 2001;204(Pt 19):3389-99.

74. Srinivasan S, Weimer DA, Agans SC, Bain SD, Gross TS. Low-magnitude mechanical loading becomes osteogenic when rest is inserted between each load cycle. Journal of bone and mineral research : the official journal of the American Society for Bone and Mineral Research. 2002;17(9):1613-20.

75. Karlsson MK, Nordqvist A, Karlsson C. Physical activity increases bone mass during growth. Food Nutr Res. 2008;52.

76. Ebeling PR, Daly RM, Kerr DA, Kimlin MG. Building healthy bones throughout life: an evidence-informed strategy to prevent osteoporosis in Australia. The Medical journal of Australia. 2013;199(7 Suppl):S1.

77. Gomez-Cabello A, Ara I, Gonzalez-Aguero A, Casajus JA, Vicente-Rodriguez G. Effects of training on bone mass in older adults: a systematic review. Sports Med. 2012;42(4):301-25.

78. Ho-Pham LT, Nguyen UD, Nguyen TV. Association between lean mass, fat mass, and bone mineral density: a meta-analysis. The Journal of clinical endocrinology and metabolism. 2014;99(1):30-8.

79. Pietrobelli A, Faith MS, Wang J, Brambilla P, Chiumello G, Heymsfield SB. Association of lean tissue and fat mass with bone mineral content in children and adolescents. Obes Res. 2002;10(1):56-60.

80. Ashby RL, Adams JE, Roberts SA, Mughal MZ, Ward KA. The muscle-bone unit of peripheral and central skeletal sites in children and young adults. Osteoporosis international : a journal established as result of cooperation between the European Foundation for Osteoporosis and the National Osteoporosis Foundation of the USA. 2011;22(1):121-32.

81. Barrera G, Bunout D, Gattas V, de la Maza MP, Leiva L, Hirsch S. A high body mass index protects against femoral neck osteoporosis in healthy elderly subjects. Nutrition. 2004;20(9):769-71.

82. Winther A, Jorgensen L, Ahmed LA, Christoffersen T, Furberg AS, Grimnes G, et al. Bone mineral density at the hip and its relation to fat mass and lean mass in adolescents: the Tromso Study, Fit Futures. BMC Musculoskelet Disord. 2018;19(1):21.

83. Golden NH. Optimizing bone health in Brazilian teens: using a population-based survey to guide targeted interventions to increase dietary calcium intake. Jornal de pediatria. 2016;92(3):220-2.

84. Winzenberg T, Shaw K, Fryer J, Jones G. Effects of calcium supplementation on bone density in healthy children: meta-analysis of randomised controlled trials. BMJ (Clinical research ed). 2006;333(7572):775.

85. Fujita T. Osteoporosis in Japan: factors contributing to the low incidence of hip fracture. Adv Nutr Res. 1994;9:89-99. 
86. Gennari C. Calcium and vitamin D nutrition and bone disease of the elderly. Public Health Nutr. 2001;4(2b):547-59.

87. Smith TJ, Tripkovic L, Lanham-New SA, Hart KH. Vitamin D in adolescence: evidencebased dietary requirements and implications for public health policy. Proc Nutr Soc.

2018;77(3):292-301.

88. Kanis JA, Johansson H, Johnell O, Oden A, De Laet C, Eisman JA, et al. Alcohol intake as a risk factor for fracture. Osteoporosis international : a journal established as result of cooperation between the European Foundation for Osteoporosis and the National Osteoporosis Foundation of the USA. 2005;16(7):737-42.

89. Lucas R, Fraga S, Ramos E, Barros H. Early initiation of smoking and alcohol drinking as a predictor of lower forearm bone mineral density in late adolescence: a cohort study in girls. PLoS One. 2012;7(10):e46940.

90. Dorn LD, Beal SJ, Kalkwarf HJ, Pabst S, Noll JG, Susman EJ. Longitudinal impact of substance use and depressive symptoms on bone accrual among girls aged 11-19 years. J Adolesc Health. 2013;52(4):393-9.

91. Wosje KS, Kalkwarf HJ. Bone density in relation to alcohol intake among men and women in the United States. Osteoporosis international : a journal established as result of cooperation between the European Foundation for Osteoporosis and the National Osteoporosis Foundation of the USA. 2007;18(3):391-400.

92. Schnitzler CM, Solomon L. Bone changes after alcohol abuse. South African medical journal = Suid-Afrikaanse tydskrif vir geneeskunde. 1984;66(19):730-4.

93. Chappard D, Plantard B, Petitjean M, Alexandre C, Riffat G. Alcoholic cirrhosis and osteoporosis in men: a light and scanning electron microscopy study. Journal of studies on alcohol. 1991;52(3):269-74.

94. LaBrie JW, Boyle S, Earle A, Almstedt HC. Heavy Episodic Drinking Is Associated With Poorer Bone Health in Adolescent and Young Adult Women. Journal of studies on alcohol and drugs. 2018;79(3):391-8.

95. Fuggle NR, Singer J, Clynes MA, Curtis B, Wyawahare P, Denison HJ, et al. Risk Factors for Fracture in Alcohol-dependent, Pre-menopausal Females. The International Journal of Alcohol and Drug Research. 2018;7(2).

96. Kanis JA, Johnell O, Oden A, Johansson H, De Laet C, Eisman JA, et al. Smoking and fracture risk: a meta-analysis. Osteoporosis international : a journal established as result of cooperation between the European Foundation for Osteoporosis and the National Osteoporosis Foundation of the USA. 2005;16(2):155-62.

97. Winther A, Dennison E, Ahmed LA, Furberg AS, Grimnes G, Jorde R, et al. The Tromso Study: Fit Futures: a study of Norwegian adolescents' lifestyle and bone health. Archives of osteoporosis. 2014;9:185.

98. Gaffney CD, Pagano MJ, Kuker AP, Stember DS, Stahl PJ. Osteoporosis and Low Bone Mineral Density in Men with Testosterone Deficiency Syndrome. Sexual medicine reviews. 2015;3(4):298-315.

99. Khan ME, Anker M, Patel BC, Barge S, Sadhwani H, Kohle R. The use of focus groups in social and behavioural research: some methodological issues. World Health Stat Q. 1991;44(3):145-9.

100. Kitzinger J. Qualitative research. Introducing focus groups. BMJ (Clinical research ed). 1995;311(7000):299-302 .

101. Malterud K. Qualitative research: standards, challenges, and guidelines. Lancet (London, England). 2001;358(9280):483-8. 
102. Krall EA, Dawson-Hughes B. Heritable and life-style determinants of bone mineral density. Journal of bone and mineral research : the official journal of the American Society for Bone and Mineral Research. 1993;8(1):1-9.

103. Holroyd C, Harvey N, Dennison E, Cooper C. Epigenetic influences in the developmental origins of osteoporosis. Osteoporosis international : a journal established as result of cooperation between the European Foundation for Osteoporosis and the National Osteoporosis Foundation of the USA. 2012;23(2):401-10.

104. Rizzoli R, Bianchi ML, Garabedian M, McKay HA, Moreno LA. Maximizing bone mineral mass gain during growth for the prevention of fractures in the adolescents and the elderly. Bone. 2010;46(2):294-305.

105. Weaver CM. Parallels between nutrition and physical activity: research questions in development of peak bone mass. Res Q Exerc Sport. 2015;86(2):103-6.

106. Oh EG, Lee JE, Yoo JY. A systematic review of the effectiveness of lifestyle interventions for improving bone health in women at high risk of osteoporosis. JBI Libr Syst Rev.

2012;10(30):1738-84.

107. Sampson HW. Alcohol and other factors affecting osteoporosis risk in women. Alcohol research \& health : the journal of the National Institute on Alcohol Abuse and Alcoholism. 2002;26(4):292-8.

108. Bielemann RM, Martinez-Mesa J, Gigante DP. Physical activity during life course and bone mass: a systematic review of methods and findings from cohort studies with young adults. BMC Musculoskelet Disord. 2013;14:77.

109. Zulfarina MS, Sharkawi AM, Aqilah SNZ, Mokhtar SA, Nazrun SA, Naina-Mohamed I. Influence of Adolescents' Physical Activity on Bone Mineral Acquisition: A Systematic Review Article. Iran J Public Health. 2016;45(12):1545-57.

110. Kalinowski A, Humphreys K. Governmental standard drink definitions and low-risk alcohol consumption guidelines in 37 countries. Addiction (Abingdon, England). 2016;111(7):1293-8.

111. Robertson K, Tustin K. Students Who Limit Their Drinking, as Recommended by National Guidelines, Are Stigmatized, Ostracized, or the Subject of Peer Pressure: Limiting Consumption Is All But Prohibited in a Culture of Intoxication. Substance Abuse: Research and Treatment. 2018;12:1178221818792414.

112. Klein RF. Alcohol-induced bone disease: impact of ethanol on osteoblast proliferation. Alcohol Clin Exp Res. 1997;21(3):392-9.

113. Seo S, Chun S, Newell MA, Yun M. Association between alcohol consumption and Korean young women's bone health: a cross sectional study from the 2008 to 2011 Korea National Health and Nutrition Examination Survey. BMJ Open. 2015;5(10):e007914.

114. Janssen MM, Mathijssen JJ, van Bon-Martens MJ, van Oers HA, Garretsen HF. A qualitative exploration of attitudes towards alcohol, and the role of parents and peers of two alcohol-attitude-based segments of the adolescent population. Subst Abuse Treat Prev Policy. 2014;9:20.

115. Yoon V, Maalouf NM, Sakhaee K. The effects of smoking on bone metabolism. Osteoporosis international : a journal established as result of cooperation between the European Foundation for Osteoporosis and the National Osteoporosis Foundation of the USA. 2012;23(8):2081-92.

116. Werner P. Knowledge about osteoporosis: assessment, correlates and outcomes. Osteoporosis international : a journal established as result of cooperation between the European Foundation for Osteoporosis and the National Osteoporosis Foundation of the USA. 2005;16(2):115-27. 
117. Fleary SA, Joseph P, Pappagianopoulos JE. Adolescent health literacy and health behaviors: A systematic review. J Adolesc. 2018;62:116-27.

118. Ndumbe-Eyoh S, Mazzucco A. Social media, knowledge translation, and action on the social determinants of health and health equity: A survey of public health practices. J Public Health Policy. 2016;37(Suppl 2):249-59. 


\section{Appendix A}

\section{Poster}

This research study was presented as poster at New Zealand Rheumatology Association Conference in Rotorua on 6th September, 2019.

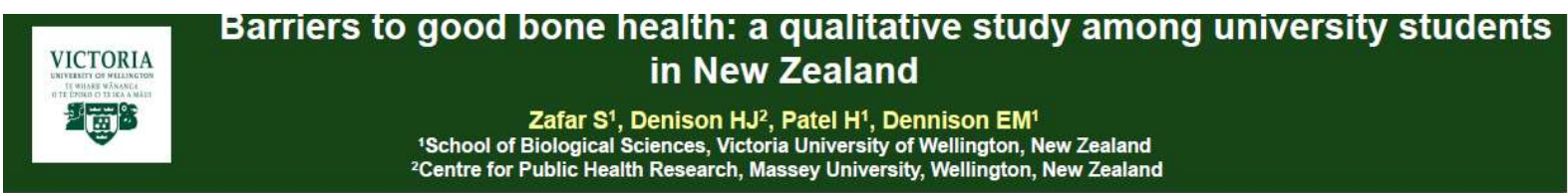

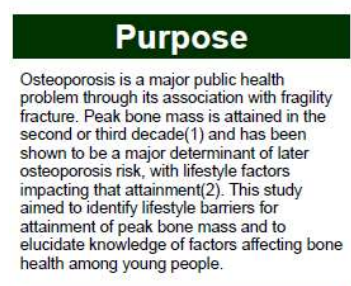

\section{Methods}

Participants : A total of 28 students (7 aged between $18-35$ years were recruited

Setting : Victoria University of Wellington, Kelburn Campus, New Zealand

Procedure : Collected data by conducting six semi structured focus group intervi

\section{Research Questions}

Our study focused on two main research questions:

\&What do adolescents and young adults usterstand about peak

$*$ What are the barriers that young people fee in adopting lifestyles associated with better bone health and how should we reduce this?
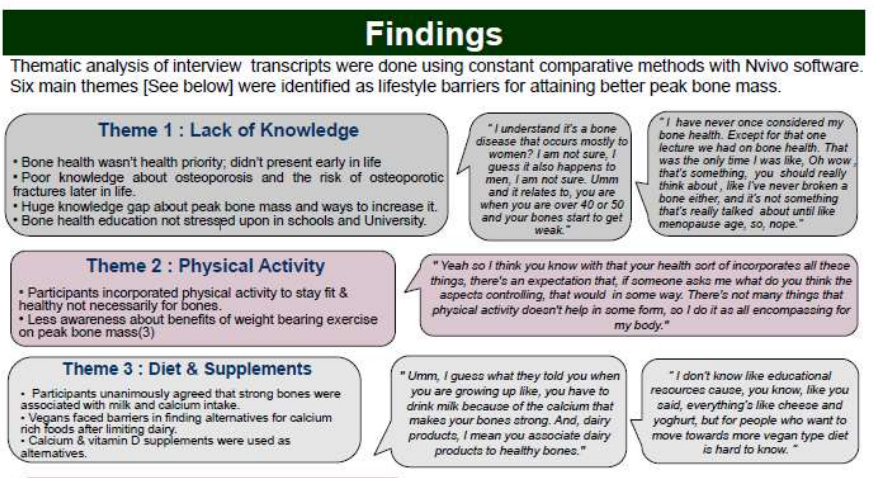

Theme 4 : Time Management

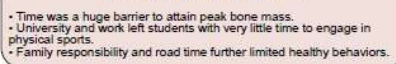
Theme 5 : Lack of Motivation

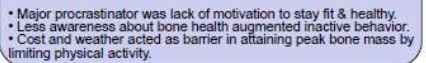

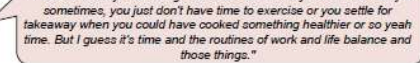
those thing.

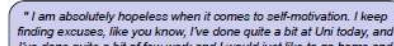

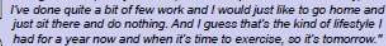

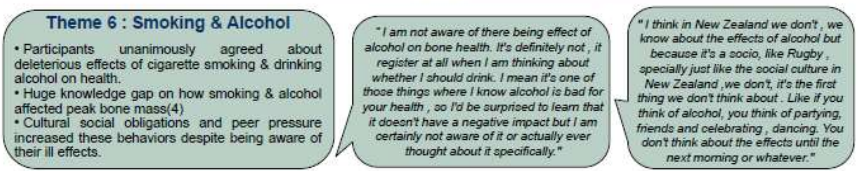

\section{Results}

Students had limited knowledge of bone health and osteoporosis. A huge knowledge gap was demonstrated with regard to peak bone mass and the lifestyle factors that could increase it here was stong molvation to keep good bone health. Bone health knowle was limited to dairy and calcium, where intake was often low.

\section{Conclusions}

These data highlights a lack of awareness of lifestyle factors that have an impact on peak bone mass of University students. Students were eager to learn more about bone health and adopt healthy lifestyles. stronger foundations with regards to knowledge of bone health. These might help design interventions that encourage optimal peak bone mass in later life in young adults.

\section{References}

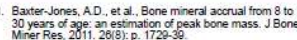

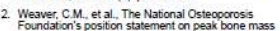

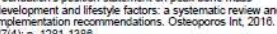

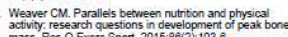

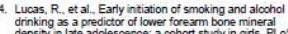


Appendix B 


\section{HDEC Approval and Information Form}

HDEC Approval: The ethics for this study were obtained as part of a larger existing study

Health
and
Disability
Ethics
Committees

11 October 2018

Mrs Hansa Patel

50 Arahiwi Grove

LOWer Hutt
WELLINGTON 5010

Dear Mrs Patel,
Health and Disability Ethics Committoes Misisy of that 133 Moeswort Sted
PO Bor 5013 Welingon

08004 ETHCS

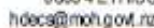

I am pleased to advise that this amendment has been approved by the Central Health and Disability Ethics Committee. This decision was made through the HDEC Expedited Review pathway.

Please dont hesitate to contact the HDEC secretariat for further information. We wish you all the best for your study.

Yours sincerely,

\section{.}

Mrs Helen Walker

Chaimerson

Central Health and Disability Ethics Committee

Encl: appendix A: documents submitted

appendix B: statement of compliance and list of members 
Appendix A

Documents submitted and approved

\begin{tabular}{|l|l|l|}
\hline Document & Version & Date \\
\hline PIS/CF: 10Sep18 qualitative study protocol others 16-35 & 2 & 10 September 2018 \\
\hline Protocol: 10Sep 18 qualitative study protocol others 16-35 & 2 & 10 September 2018 \\
\hline 10Sept18 Recruitment Flyer & 1 & 10 September 2018 \\
\hline PIS/CF: Tracked changed on PDF & 2 & 10 September 2018 \\
\hline Protocol: Tracked changes on PDF & 2 & 10 September 2018 \\
\hline Post Approval Form & AM03 & 20 September 2018 \\
\hline
\end{tabular}




\section{Appendix B}

Statement of compliance and list of members

\section{Statement of compliance}

The Central Health and Disability Ethics Committee:

- is constituted in accordance with its Tems of Reference

- operates in accordance with the Standard Operating Procectures for Heath and Disability Ethics Committees, and with the principles of intemational good clinical practice (GCP)

- is approved by the Health Research Council of New Zealand's Ethics Cormittee for the purposes of section 25(1)(c) of the Health Research Council Act 1990

— is registered (number 00008712) with the US Department of Health and Human Services' Office for Human Research Protection (OHRP).

List of members

\begin{tabular}{|c|c|c|c|}
\hline Name & Category & Appointed & Term Expirs \\
\hline Mrs Haien Waker & $\begin{array}{l}\text { Lay (ponsumerloommurity } \\
\text { perspectives) }\end{array}$ & $0107 / 2015$ & 01072018 \\
\hline Dr Peter Galagier & $\begin{array}{l}\text { Non-lay (heativdisabiaty servica } \\
\text { provision) }\end{array}$ & $30 / 072015$ & $30.07 / 2018$ \\
\hline Ms Sandy Gill & $\begin{array}{l}\text { Lay foonsumerioommuniy } \\
\text { perspoctives) }\end{array}$ & $30.07 / 2015$ & $30 / 07 / 2018$ \\
\hline Dr Patifes Herst & Nan-lay (irtamerifon studes) & $27 / 102015$ & $27 / 102018$ \\
\hline Dr Dean Quim & Nortiay (irferventon studies) & $27 \pi / \sqrt[2015]{2015}$ & $27 / 102018$ \\
\hline Dr Cordelia Thomas & Lay the law? & $20,0 5 \longdiv { 2 0 1 7 }$ & $20,05 / 2020$ \\
\hline
\end{tabular}

Uness members resign, vacate or are removed from their office, every member of HDEC shall continue in office until their successor comes into office (HDEC Terms of Reference)

\section{http:/lwww.ethics. health.govtnz}


INFORMATION SHEET FOR PARTICIPANTS AGED 16

TO 35 INCLUSIVELY

Please go through this information sheet and make sure you understand what is involved. Please contact me if vou have anvauestions.

Thank you for your interest in this study. Please read this information before deciding whether or not you would like to take part.

\section{What is the Research?}

- We would like to find out how young people feel about the lifestyle choices that are associated with better bone health later in life. If we can identify the barriers to adoption of a lifestyle that leads to better bone health, we can try to reduce those barriers.

\section{Why have you been asked to take part?}

- We are asking young people aged 16 to 35 from all walks of life to share with us your views in a one-off interview on different factors in day to day life which have an impact on bone health positively or negatively. The team would like to take your opinion and discuss various contributing factors such as what sorts of physical activity you engage in (jogging, cycling, sports etc.), the type of food you consume, etc.

- Even if you feel like you have nothing to say, we would love to hear from you. Everyone's contribution will enable us to make a positive difference to bone health in New Zealand.

\section{What does the study involve?}

- We would like you to take part in a discussion with other young people about factors that impact the lifestyle choices you make now that might impact bone health later in life.

- If you participate in this study, you will be in a group of approximately 4-10 young people. There will be a facilitator who will ask questions and facilitate the discussion, and a note- taker to write down the ideas expressed within the group. The focus group will last about 45 minutes and will be audiotaped.

\section{Voluntary Participation}

- This discussion is voluntary - you do not have to take part if you do not want to.

- If any questions make you feel uncomfortable, you do not have to answer them.

- You may leave the group at any time for any reason without consequence. Please note that since data will be collected and stored in de-identified form, any data that you have provided will not be able to be withdrawn.

\section{Privacy}

- Your privacy will be protected.

- Your name will not be used in any report that is published.

- The discussion will be kept strictly confidential. 
- The other young people in the group will be asked keep what we talk about private, but this cannot be assured.

- All research data (including the audio recordings and paper data) will be stored in a secure facility at the School of Biological Sciences, Victoria University of Wellington (VUW) and on a password protected computer for ten years, and then destroyed.

- This research is confidential. This means that the researchers named below will be aware of your identity but the research data will be grouped together and your name will not be shared with anyone else.

- A summary of the results of the study will be available for viewing at the VUW library as part of a PhD thesis, if requested a summary may be provided.

Payment: You will receive a grocery voucher as a token of appreciation for taking part in the focus group.

Questions: We will be happy to answer any questions you have about this study. If you have further questions about this project or if you have a research-related problem, you may contact the coordinating researcher by email Hansa.Patel@vuw.ac.nz

Ethical approval: This project forms the basis of a VUW PhD studentship and has been reviewed and approved by the VUW Human Ethics Committee (ref: 023752) and the Health and Disability Ethics Committee (HDEC) 18/CEN/18.

\section{Contact information: \\ Supervisor}

Name: Professor Elaine Dennison (Clinical Research)

School: Biological Sciences Phone: +64 44635339

Email: Elaine.Dennison@vuw.ac.nz

\section{Human Ethics Committee Information}

If you have any concerns about the ethical

conduct of the research you may contact:

Name: Dr Judith Loveridge

Position: VUW HEC Convener

Phone: +64 44636028

Email: hec@vuw.ac.nz

\section{Other Support}

For Maori support please contact:

Name: Associate Professor Meegan Hall

(Ngāti Ranginui, Ngāti Tūwharetoa)

Position: Assistant Vice-Chancellor

(Mātauranga Māori)

Phone: +64 44635793

Email: meegan.hall@vuw.ac.nz

If you want to talk to someone who is not involved with the study, you can contact an independent health and disability advocate on:

Phone: 0800555050

Fax: 08002 SUPPORT (o80o 27877678 )

Email: advocacy@hdc.org.nz

You can also contact HDEC on:

Phone: 08004 ETHICS

Email: hdecs@moh.govt.nz 


\section{WRITTEN CONSENT TO PARTICIPATE FOR PARTICIPANTS AGED 16 to 35 inclusively}

Researcher: Dr.Sana Zafar, Masters in Clinical Research candidate

Name of supervisor: Prof Elaine Dennison

\section{This project has been approved by the VUW Human Ethics Committee (ref: \#023752) and HDEC 18/CEN/18.}

- I have been given the Participant Information Sheet and understand the information provided in it.

- I understand that my participation in this study will involve me taking part in a focus group with other young people.

- I consent to the audio-recording and transcribing of the focus group discussion. All data will be de-identified and stored in a locked filing cabinet or on a password protected computer.

- I understand that data collected during the study may be shared with researchers in New Zealand and in other countries (that may have different privacy laws to New Zealand). I understand that researchers will not be given my name or any other information that would enable them to identify me. I understand that the results will be used for a $\mathrm{PhD}$ report and a summary of the results may be used in academic reports and/or presented at conferences.

- I understand that data will be stored securely, and will be kept for up to ten years after study completion.

- I understand that my participation is voluntary and that I can leave the group at any time for any reason without consequence. I understand any data provided will not be able to be withdrawn but will be stored in de-identified form.

Participant's Name

Date of Birth

Contact Email

Signature

Date

Health and Disability Ethics Committee Ref: 18/CEN/18 (VUW Human Ethics Committee \#023752)

Sub-study title: Barriers to adopting a better lifestyle for bone health later in life - Focus Group Study Focus Group a果里 16 to 35 


\section{Appendix C}

\section{Invitation Letter}

Kia Ora,

We are writing to invite you to participate in focus groups for our research project "Barriers to a lifestyle associated with better Peak Bone Mass".

The research team is inviting participants to tell us their views in a one-off interview about different factors in day to day life which have an impact on bone health positively or negatively. The focus group will be audio taped and is expected to last approximately 60 minutes.

The information participants give us in these interviews will be used to help the research team to explain the results of the study more fully and to give other people in the same position as yourself, information to help them decide which factors will be beneficial to their bone health. There are no right or wrong answers to this and we are keen to gain a wide variety of opinions.

Your name will not appear in any publication and it will not be shared with anyone outside of the research team. I've attached an information sheet about the study, as well as the consent form which we can go through on the day of the focus group together if you wish to take part.

The interviewees will receive a $\$ 20$ gift voucher for their participation.

If you have any questions about the study then please do contact Dr. Sana Zafar on zafarsana@myvuw.ac.nz who will be happy to discuss with you any questions you may have.

Thank you very much for reading this email.

Ngā mihi,

Dr. Sana Zafar
Research team members:

Hansa Patel PhD. Candidate

Dr. Hayley Denison

Prof. Elaine Dennison

Dr. Sana Zafar

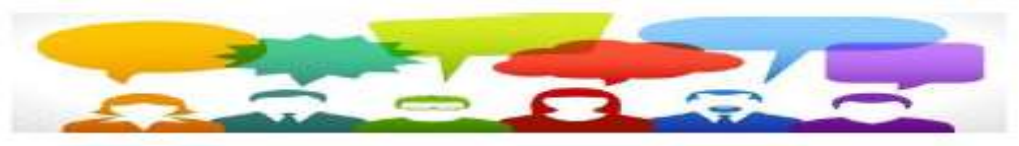




\section{Appendix D}

\section{Project Risk Assessment}

Safety and risk must be considered in the planning and design of all research projects at VUW. The purpose of a risk assessment is to provide an opportunity for you to review your project methodology and ensure you have identified all of the hazards that may be encountered. A risk assessment must be completed when you are preparing a project proposal and/or before you begin a new piece of research. Your supervisor must check and approve your risk assessment before you begin any research activities.

What is a Risk Assessment? A hazard is any condition or circumstance that could lead, or contribute, to an undesirable event or cause harm. A risk assessment is when you identify the potential problems (hazards) that could arise in the environment you are operating in. This includes the failure or malfunction of equipment and systems. A risk matrix is used to give each hazard a risk rating - the higher the rating the more significant the risk. In some situations an identified hazard can be eliminated or isolated. However, there will be times that a certain degree of risk is acceptable if the hazard is minimized by equipment, assistance, training, behavioural changes, etc. The steps you take to eliminate, isolate or minimize a hazard are called 'controls'. The controls should also include the emergency procedures that will be followed if there is an accident or incident. When planning to control a particular hazard, it is important to consider the possibility of it combining with other hazards and/or conditions. Accidents and incidents most often occur as a result of a sequence of events. Give particular attention to any hazards that have the potential to cause serious harm and a high likelihood of occurring. When the controls have been put in place the risk matrix is used again to determine the residual risk of the hazard. The goal is to use all reasonably practicable controls to reduce the residual risk of a hazard to a low and acceptable level.

A research project will not be approved unless all reasonably practicable controls have been put in place to reduce risks to a safe level (seek advice on acceptable levels of risk if you are unsure). The initial risk assessment of a project might identify hazards that require the controls to be properly validated by a more thorough risk analysis. The levels of risk will vary significantly among projects depending on what sort of research is proposed. The sorts of hazards encountered during projects in SBS can range from 
conducting relatively deep-water dives, handling highly toxic chemicals, to extended periods of computer use (e.g. repetitive strain injury). In some cases, particular medical condition(s) (e.g. allergic reaction, diabetic, etc) of a participating individual might increase the level of risk associated with a hazard. This would require specific controls (including emergency plans) to be established in order to reduce risk to an acceptable level. Any discloser of a medical condition on the risk assessment form will be treated as confidential in accordance with the University's privacy policy.

When you are preparing your thesis research proposal, work together with your supervisor to complete a risk assessment for your project. For field work (Diving, Boating, and off-campus terrestrial activities) use the Fieldteq system ( https://fieldteq.vuw.ac.nz/ ), for laboratory projects use the excel spreadsheet Risk Register ( http://www.victoria.ac.nz/healthandsafety/risk-management ). Upon completion of your risk assessment, and approval by your supervisor, please attach it to your project proposal. Your risk assessment might need to be updated at a later stage if you change the project methodology. Every year you should review your risk assessment to keep it up-to-date.

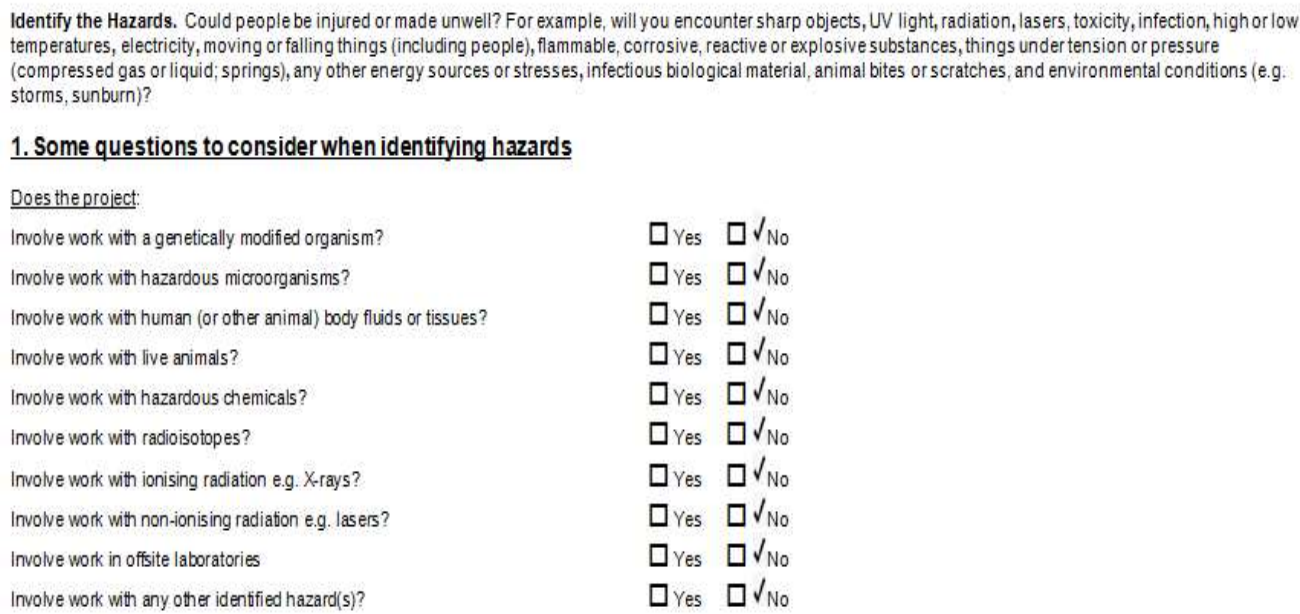

\section{ChemWatch: Information about Chemical Hazards and Controls}
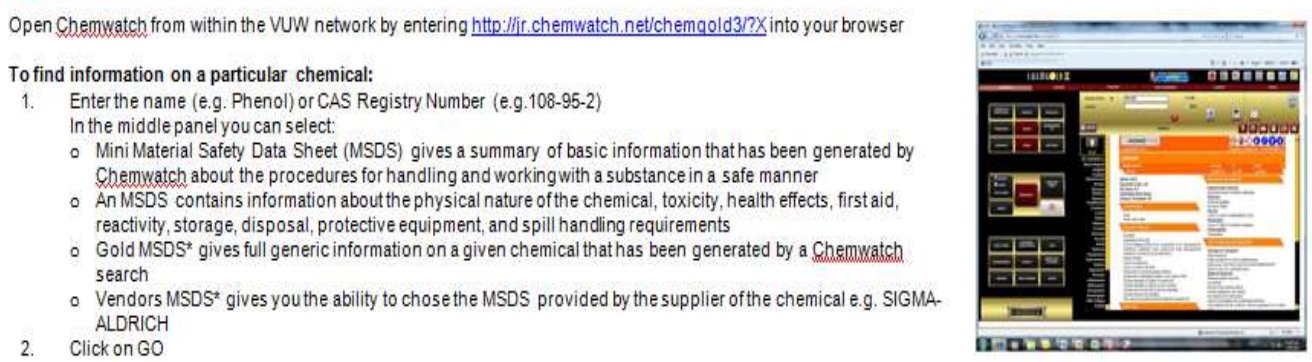

No risks were identified for the project. 


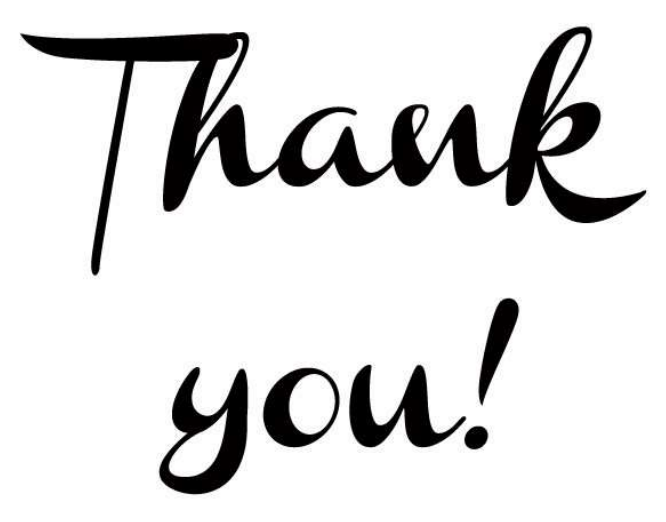

